\title{
Sketch and Learn: a sketch-based game framework for studying images
}

by

\author{
Rajinder Wasson
}

\author{
A Thesis submitted to \\ the Faculty of Graduate Studies and Research \\ in partial fulfilment of \\ the requirements for the degree of \\ Master of Computer Science \\ in
}

\author{
Computer Science \\ School of Computer Science \\ Carleton University \\ Ottawa, Ontario, Canada \\ Summer 2012
}

Copyright (C)

2012 - Rajinder Wasson 
Library and Archives

Canada

Published Heritage

Branch

395 Wellington Street

Ottawa ON K1A ON4

Canada
Bibliothèque et

Archives Canada

Direction du

Patrimoine de l'édition

395 , rue Wellington

Ottawa ON K1A ON4

Canada
Your file Votre référence

ISBN: 978-0-494-93706-8

Our file Notre référence

ISBN: $978-0-494-93706-8$
NOTICE:

The author has granted a nonexclusive license allowing Library and Archives Canada to reproduce, publish, archive, preserve, conserve, communicate to the public by telecommunication or on the Internet, loan, distrbute and sell theses worldwide, for commercial or noncommercial purposes, in microform, paper, electronic and/or any other formats.

The author retains copyright ownership and moral rights in this thesis. Neither the thesis nor substantial extracts from it may be printed or otherwise reproduced without the author's permission.
AVIS:

L'auteur a accordé une licence non exclusive permettant à la Bibliothèque et Archives Canada de reproduire, publier, archiver, sauvegarder, conserver, transmettre au public par télécommunication ou par l'Internet, prêter, distribuer et vendre des thèses partout dans le monde, à des fins commerciales ou autres, sur support microforme, papier, électronique et/ou autres formats.

L'auteur conserve la propriété du droit d'auteur et des droits moraux qui protege cette thèse. $\mathrm{Ni}$ la thèse ni des extraits substantiels de celle-ci ne doivent être imprimés ou autrement reproduits sans son autorisation.
In compliance with the Canadian Privacy Act some supporting forms may have been removed from this thesis.

While these forms may be included in the document page count, their removal does not represent any loss of content from the thesis.
Conformément à la loi canadienne sur la protection de la vie privée, quelques formulaires secondaires ont été enlevés de cette thèse.

Bien que ces formulaires aient inclus dans la pagination, il n'y aura aucun contenu manquant. 


\section{Abstract}

Art history students see hundreds of different images and must memorize the significant details of each one. Their learning would be increased by combining the pleasures and addictive properties of a video game with a mechanism to interact with the images while learning the details. This thesis describes the design and implementation of an educational framework, called Sketch and Learn, that applies effective game mechanics to motivate the player to spend more time viewing images and remember the accompanying details. We had 25 students participate in a game using Sketch and Learn images and textbook-style images. Our results showed that participants spent three times more time on Sketch and Learn images and remembered $60 \%$ more of their details. The Sketch and Learn framework has great potential as a learning tool to help students of any language develop skills and knowledge in any subject matter that involves images. 


\section{Table of Contents}

$\begin{array}{ll}\text { Abstract } & \text { ii }\end{array}$

Table of Contents $\quad$ iii

List of Tables $\quad$ vi

List of Figures $\quad$ vii

1 Introduction $\quad 1$

1.1 Thesis Problem and Objective .................. 2

1.2 Thesis Outline . . . . . . . . . . . . . . . . 3

1.3 Thesis Contributions . . . . . . . . . . . . . . . . 4

2 Theoretical Framework 5

2.1 Practices for Art History . . . . . . . . . . . . . . . . 5

2.2 Video Games and Education . . . . . . . . . . . . . . 7

2.2 .1 History ...................... 8

2.2 .2 Integration . . . . . . . . . . . . . 11

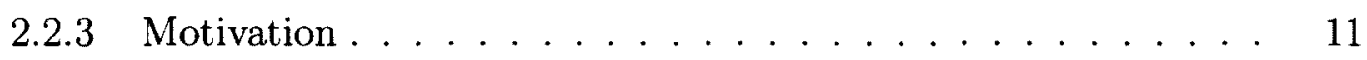

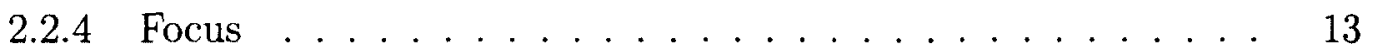

2.2 .5 Flow ............................... 14

2.2 .6 Achievements ...................... 17 
2.2 .7 Game Mechanics . . . . . . . . . . . . . . . . . 18

2.3 Visual Attention and Memory . . . . . . . . . . . . . . . 23

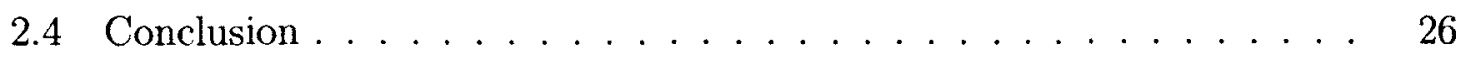

3 The Design of Sketch and Learn 27

3.1 Controllers . . . . . . . . . . . . . . . . . . . 28

3.2 Player Hub . . . . . . . . . . . . . . . . . . . . . . 29

3.3 Game View . . . . . . . . . . . . . . . . . . . 30

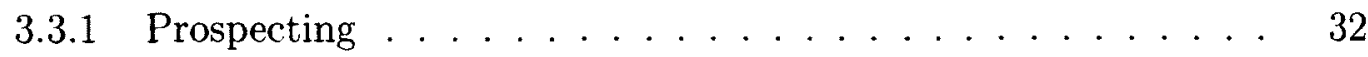

3.3 .2 Glyphs . . . . . . . . . . . . . . . . 34

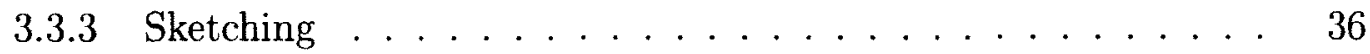

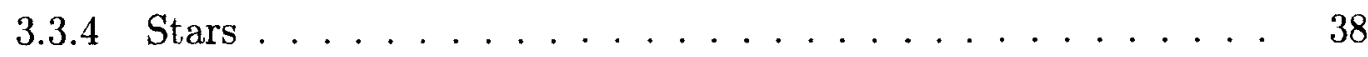

3.3 .5 Fact Delivery $\ldots \ldots \ldots \ldots \ldots \ldots \ldots$

3.4 Limitations . . . . . . . . . . . . . . . . 41

3.5 Summary $\ldots \ldots \ldots \ldots \ldots \ldots \ldots \ldots \ldots \ldots \ldots$

4 Study Design $\quad 44$

4.1 The Game . . . . . . . . . . . . . . . . . . 44

4.2 Hypotheses . . . . . . . . . . . . . . . 46

4.3 Methods . . . . . . . . . . . . . . . . 47

4.3 .1 Participants . . . . . . . . . . . . . . . 48

$4.3 .2 \quad$ Experimental Groups . . . . . . . . . . . . . . . . . 48

4.3 .3 Test Session Overview $\ldots \ldots \ldots \ldots$

$4.3 .4 \quad$ Test Session Detail . . . . . . . . . . . . . . . . . 49

4.3.5 Data Collection . . . . . . . . . . . . . 50

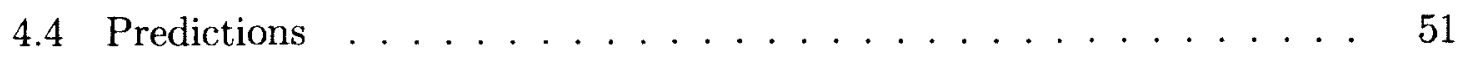


5 Results and Analysis $\quad 53$

5.1 Time Spent . . . . . . . . . . . . . . . 55

5.2 Facts Recalled . . . . . . . . . . . . . . . . . 74

5.3 Questionnaire and Discussion ... . . . . . . . . . 83

5.4 Summary .............................. 84

$\begin{array}{llr}6 & \text { Conclusion } & 86\end{array}$

6.1 Future Work . . . . . . . . . . . . . . . . 87

$\begin{array}{ll}\text { A Art History Images Used in Study } & 89\end{array}$

$\begin{array}{ll}\text { B Questionnaires } & 96\end{array}$

$\begin{array}{ll}\text { List of References } & 99\end{array}$ 


\section{List of Tables}

1 Style of images for each group . . . . . . . . . . . . 49

2 Comparison of Time in Minutes between Game Style and Textbook Style 55

3 Glyph Facts recalled for both images . . . . . . . . . . . . . 76

4 Glyph Facts recalled for Image $1 \ldots \ldots \ldots \ldots$

$5 \quad$ Glyph Facts recalled for Image $2 \ldots \ldots \ldots \ldots . \ldots \ldots$ 


\section{List of Figures}

1 The flow channel (adapted from Csikszentmihalyi, 1997) $\ldots \ldots .16$

2 A screenshot of the Minesweeper game . . . . . . . . . . . . 19

3 A screenshot of the Mass Effect 2 scanning mechanic . . . . . . . 19

4 A screenshot of the game nuWar that uses a sketch mechanic and shows the player describing an ambush $\ldots \ldots \ldots \ldots 21$

5 A screenshot of the players hub . . . . . . . . . . . . 29

6 A schematic of the Sketch and Learn game page . . . . . . . . . 30

$7 \quad$ A screenshot of the full Sketch and Learn game page . . . . . . . . 31

8 Screenshots of the prospecting mechanic . . . . . . . . . 32

9 Prospecting and discovering a glyph $\ldots \ldots \ldots \ldots \ldots \ldots$

9 Types of glyphs $\ldots \ldots \ldots \ldots \ldots \ldots \ldots \ldots \ldots \ldots \ldots$

10 Sketching a glyph $\ldots \ldots \ldots \ldots \ldots \ldots \ldots \ldots \ldots$

11 A screenshot of a complete glyph . . . . . . . . . . 39

12 Comparison of the styles $\ldots \ldots \ldots \ldots \ldots \ldots \ldots$

13 Breakdown of how often participants play video games $\ldots \ldots \ldots 54$

14 Histogram of previous knowledge $\ldots \ldots \ldots \ldots \ldots \ldots$

15 Total average time spent on images for all participants . . . . . 56

16 Histogram for Sketch and Learn times . . . . . . . . . . . 57

17 Histogram for Textbook times . . . . . . . . . . . . . 57

18 Total average time for art history and non-art history participants . . 58 
19 Average time by number of details/glyphs $\ldots \ldots \ldots \ldots$

20 Histogram of standard scores separated by style . . . . . . . . . 61

21 Image of the Calling of Saint Matthew . . . . . . . . . 62

22 A hot spot image of the Calling of Saint Matthew Sketch and Learnstyle page $\ldots \ldots \ldots \ldots \ldots \ldots \ldots$

23 A hot spot image of the Saint Matthew textbook-style page . . . . 64

24 A hot spot comparison of The Calling Saint Matthew . . . . . . . 64

25 A hot spot comparison of The Calling Saint Matthew (normalized for overall time $\ldots \ldots \ldots \ldots \ldots \ldots \ldots \ldots$

26 A hot spot comparison of The Calling of Saint Matthew (normalized for overall time and textbox time) $\ldots \ldots \ldots \ldots \ldots 6$

27 A hot spot comparison of the Calling Saint Matthew with the glyph locations . . . . . . . . . . . . . . . 67

28 Breakdown of time spent for participant $1 \ldots \ldots \ldots 68$

29 Image viewed by participant $2 \ldots \ldots \ldots \ldots \ldots \ldots$

30 Breakdown of time spent for participant $2 \ldots \ldots \ldots \ldots$

31 Image viewed by participant $3 \ldots \ldots \ldots \ldots \ldots$

32 Breakdown of time spent for participant $3 \ldots \ldots \ldots 71$

33 Image viewed by participant $4 \ldots \ldots \ldots \ldots \ldots$

34 Breakdown of time spent for participant $4 \ldots \ldots \ldots 72$

35 A scatter plot for time and glyph facts . . . . . . . . . 77

36 A scatter plot showing lines of best fit without outlier . . . . . . 79

37 A scatter plot for time spent and glyph facts recalled. One line is drawn for each participant, linking their two scores . . . . . . . 80

38 A scatter plot for time and all facts spoken . . . . . . . . . . . 81

39 A scatter plot for time spent and all facts described. One line is drawn for each participant, linking their two scores $\ldots \ldots \ldots \ldots$ 
40 Holy Trinity (Masaccio) f . . . . . . . . . . . . . . . . . . 89

41 The Calling of St Matthew (Caravaggio) . . . . . . . . . . . . . 90

42 School of Athens (Raphael) . . . . . . . . . . . . . 90

43 The Arnolfini Portrait (van Eyck) . . . . . . . . . . . . 91

44 The Ambassadors (Holbein) . . . . . . . . . . . . . . 91

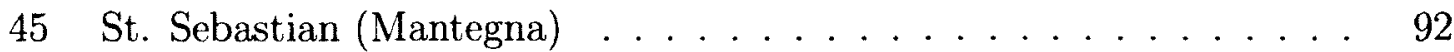

46 Mr. and Mrs. Andrews (Gainsborough) . . . . . . . . . . 93

47 The Art of Painting (Vermeer) . . . . . . . . . . . . 93

48 The Arcadian Shepherds (Poussin) . . . . . . . . . . . . . 94

49 The Oath of the Horatii (David) . . . . . . . . . . . . 94

50 A Bar at the Folies-Bergère (Manet) . . . . . . . . . . . . 95

51 Background Questionnaire . . . . . . . . . . . . . 96

52 Post-Game Questionnaire . . . . . . . . . . . . 97 


\section{Chapter 1}

\section{Introduction}

Educators have been interested in harnessing video games for educational purposes. Video games have the ability to captivate attention for both short bursts of time and extended periods as players master gameplay and complete complicated tasks. Many allow players to do something that they are normally not able to do, for instance, travelling to an exotic land to search for treasure or rescue a princess. Not only can they be part of a story, they can also influence or change the outcomes. All ages, but especially teenagers and young adults, are fascinated by anything to do with virtual realities, fast-paced action, and challenging puzzles.

For these reasons, the academic community is looking to gaming concepts to create effective and engaging educational games. Interactive games have the potential to help students learn material and skills in a natural way since they tend to learn better by doing [43]. Players would be involved in a more active experience compared to other forms of learning such as reading textbooks or listening to a lecture.

Because of the ability of video games to focus attention, they can be especially useful in teaching boring facts or completing repetitive simple tasks. A good example of a domain that requires knowledge of a large body of facts and details is art history. Students in this discipline see hundreds of different works of art over the course of their degree and must memorize the details and significance of each one. There are 
many small elements and points of interest in any given image as well as background information that is required to understand the historical and artistic significance. Students must spend significant time learning image after image, which can be boring. Most students are not willing to spend enough time on each image to remember and analyze the details.

In art history, therefore, there is a need for a learning tool that would help students learn the relevant details about works of art. A video game would seem to be ideally suited for helping students spend more time learning and studying visual images. A review of art history teaching and learning practices shows a reliance on textbooks and lecture methods, and a lack of interactive technologies.

\subsection{Thesis Problem and Objective}

The main focus of this thesis is on how we can use game concepts to get students to spend more time on studying images, and also help them remember the facts and details from those images. We propose that, by using a combination of effective game mechanics, we will be able to extend a person's viewing time on images and that they will remember more details because of it. In particular, our main goal is the development and testing of Sketch and Learn, a framework to create educational games for studying art history images.

To create this framework, we review the concepts and game mechanics related to focusing attention, motivating, and interacting. We explore the potential of these mechanics for creating an effective educational framework. Of especial interest is incorporating a challenging way for players to interact and to give them some degree of control. We also take into consideration how a person perceives visual elements and remembers. 
Our learning interface incorporates elements such as responsive controls and challenging, yet not too difficult, gameplay to keep players engaged and motivated. In particular, we design a game element called a glyph and mechanics called prospecting and sketching. Our glyph element is an outline of a mark, symbol, or area of the image which is associated with important information and facts, such as the use of a dog in the work of art to symbolize fidelity (see Appendix A 43). The glyphs are hidden in the image and the player must search for these glyphs by clicking on areas of interest. This searching is called prospecting. The player then traces the glyph to unlock the information; this is called sketching.

To test Sketch and Learn, we create a game with content provided by an art history professor. The game contains interactive Sketch and Learn images interspersed with static textbook-style images. After 25 participants play the game, we analyze their data to assess the success of Sketch and Learn and the game mechanics that were used.

\subsection{Thesis Outline}

Following this introductory chapter, we present in Chapter 2 the theoretical frameworks required when designing an educational art history game. We discuss the learning techniques and technologies that are traditionally used in art history, and the role that technology could play. We examine the crucial factors of designing educational games after looking at the history of educational video games. Some key game mechanics to help study art history are highlighted. Finally, since our game involves images, we consider how visual attention and memory affects players.

Chapter 3 covers the design of the Sketch and Learn framework and the gameplay, including the sketching and prospecting mechanics. We also indicate the techniques used to get players to spend more time observing and learning images, and we touch 
on how we chose to deliver the content to the player, and some limitations of this chosen design. Chapter 4 describes the game we developed to assess the Sketch and Learn framework. We outline our hypotheses, describe the method, and make some predictions that are tested through the in-depth analysis of the results in Chapter 5. Finally, Chapter 6 presents our conclusions as well as a look ahead to possible future studies.

\subsection{Thesis Contributions}

While exploring an art history educational video game, this thesis makes the following original contributions:

- An educational framework, called Sketch and Learn, for studying images in art history that applies effective game mechanics, such as prospecting and sketching, to motivate the player to spend more time viewing the art and remember the accompanying facts and details.

- The Sketch and Learn framework, a non-language-based learning tool that can be used for developing skills and knowledge with any subject matter that involves images, for example, anatomy, biology, or geography.

- Results from the study we performed to test the effectiveness of the mechanics used in the Sketch and Learn framework. The results show that players spend three times as much time viewing the images than the traditional textbook style and also recalled $75 \%$ more of the significant facts. 


\section{Chapter 2}

\section{Theoretical Framework}

This chapter provides the theoretical framework of the concepts used in this study. In particular, we give some background on the typical learning practices and examples of the technologies available for learning art history. It becomes evident that an interactive technology such as a game would be a valuable learning tool in art history. Then, in a brief history of educational video games, we consider some of the issues that affect educational games. These issues allow us to highlight the important factors for successful learning games and point out relevant game mechanics that are extremely effective in popular commercial games. Two elements of a good educational game are visual attention and memory, concepts which are presented in the final section of the chapter.

\subsection{Practices for Art History}

This section looks at the general learning practices that students and art historians have used to study the topic, including some of the technologies and games that exist

for learning art history. A review of the practices and technologies shows that there is a place for a new technology that features sketching and interacting with art images. 
Art history courses tend to be based on traditional textbooks that feature images of art and the history and analysis of the images in text. The principal method of teaching is the lecture method, as Joanne Sowell, a professor of art history, explains [45]. Students listen while the teacher discusses slides of images by various artists. Although some textbooks may include compact discs that have extra content such as videos and quizzes, the primary focus of the learning is the written text. Sowell points out that students often leave introductory classes with the ability to only repeat the thoughts of the teacher. They have not learned the principles of analyzing art pieces and are unable to extend what they have learned about a particular piece of art to other similar ones.

Sowell states that students need to learn how to approach art in a new way, using the process of exploration, invention, and application. For exploration, Sowell had students manipulate, sort, and order concrete objects while challenging the students to develop new modes of thinking. Students had to use the new modes of thinking to invent an explicit concept for solving problems. Finally, students had to solve new problems by applying their invented concepts. Technologies, especially games, can bring these new ways to learn closer to the students. For instance, a game could encourage the students to explore art images in their own way and apply concepts learned early in the game to later images.

The technologies that do exist for learning art history involve the same techniques discussed above, including viewing images and videos, reading descriptions and listening to audio recordings. An example is the Google Art Project [27], developed by the technology giant, which allows users to view 30000 high-resolution images from art galleries around the world. Users view the art by selecting either the location, the gallery, or the artists; users also have the ability to build their own collections to share online. The only novel approaches that Google incorporates is the super highresolution images that allow users a closer look at the brushstrokes and the ability to 
virtually walk through the art galleries and view images as they are laid out by the galleries.

Another example comes from Le Louvre, a prestigious museum located in France. The museum offers guided tours and audio guides to help learn and understand the art. The website for the museum has several "Closer Look" features for a few of the images, which include a narrated video analyzing details that are highlighted in the video [1].

As for games for learning art history, the ones that do exist do not have interaction with the image; they are either quiz-type games that test knowledge or adventure games that attempt to teach while telling a story. An example is a game by Eduweb called Pintura [20], an art history mystery where the player is tasked with investigating a painting and has to determine who the author is by comparing the style, composition, colour and subject matter.

Some art historians have found that sketching, drawing or recreating the art is helpful for learning and remembering the important information, or to them develop visual ideas. Fish and Scrivener [25] also explain how sketching is important in mental imagery and how both are linked to memory. In fact, self-help websites such as About.com [2] suggest sketching images to help remember important details and features when making notes for a painting or a drawing. In other words, physical interaction with an image can help focus a student's attention on it and can help him remember it.

\subsection{Video Games and Education}

The preceding review of art history teaching and learning shows a lack of interactive technologies to help students learn to recognize and analyze significant features and facts about works of art. Games like Rock Band [4] and Guitar Hero [3] get the player 
to play popular music by interacting with controllers modeled after musical instruments. This type of active interaction helps players learn the words and melodies to these popular songs without playing a real instrument. In our opinion, a student can learn song lyrics more quickly by trying to sing along than by passively listening to a recorded performance.

Using technology to enhance education is trending stronger now than ever before. The mobile computing power of phones, tablets and laptops is continuing to grow while the price remains affordable, enabling more people and schools to have access to them. These technologies are providing access to games and social networks for more people in more places than ever before. About $97 \%$ of American teenagers between the ages of 12 and 17 play some sort of game [15].

Commercial games also have many useful concepts that can be applied to educational games. Games like Mass Effect and The Elder Scrolls: Skyrim are massive games that have as much as 100 hours of playing time, yet manage to keep players focused and interested. Researchers are interested in how these games motivate people to spend so much time on them [14]. For these reasons, it makes sense to explore the power of video games for use in education.

\subsubsection{History}

Educational computer games have a history that dates back to at least the 1960s [22], although learning games have surely existed throughout history. Computers and video games have added the ability to create settings and scenarios that were previously difficult to simulate and eliminate some of the dangers that reality poses to a player. Military organizations around the world have had a large impact because games and simulations have been tested to replicate strategies and tactics used in battle [21]. For our purposes, a simulation is a representation of the "operation of a real-world process or system over time"; [6] ; a simplified model executes on a computer, producing 
different outputs in response to different inputs. A good example is a flight simulator, used by many to learn to fly airplanes. Games and simulations have been used for such purposes as training high-level strategy and specific cognitive team-oriented skills. In particular, the US military, one of the leaders in using simulations for training, spent over $\$ 3.0$ billion acquiring products and services for training [5].

The military wants to create scenarios with games and teach people to work together towards a common goal. These scenarios are also used to learn the behavioural patterns and cognitive skills needed to accomplish different types of missions [5]. The military's success in training through games and simulation promotes more funding and research. The problem with military research is that it is not always available to the academic community due to security reasons.

Educational institutions have many of the same learning needs as the military: to transfer content and skills to an individual in an efficient way. When we talk about non-military educational games, we tend to think of low-budget games with basic gameplay that attempt to apply simple learning theories. These games are also criticized for failing to integrate learning and game experience [22].

Another area that has had an impact on educational games is businesses and business schools. Corporations have used simulations in everything from forecasting the economy to helping them understand behaviours of complex systems [29]. Some corporations have used games for training management techniques, and business schools are using games to teach just as often as they use case studies [24]. Although these business-oriented games (for example, emergency management, flight simulators, weather forecasters) have made an impact commercially, they are still not as popular as shooting, action or sports-based games.

Most of the research on using technology to learn relates to other forms of educational media such as television and film, but can be applied to games [21]. Even though the research is useful, it is important to note that these are one-way types 
of media, where the person viewing does not have to interact with it. Games are interactive by nature and require the person to interact with them in different ways.

Media such as films, television and games that combine education with entertainment have been called "edutainment." Buckingham [10] explains that edutainment "relies heavily on visual material, on narrative or game-like formats, and on informal, less didactic styles of address." Edutainment games tend to have a low amount of interactivity and follow a linear type of progression, preventing players from wandering or deviating from the main path [17]. Another characteristic is a poor connection between gameplay and what the player is supposed to learn [22]. In other words the main game mechanics are separate from the learning aspects of the game. For instance, in a game teaching math, rapidly pressing buttons to make a character move and fight, does not help the player learn math.

Nielsen [22] states that part of the problem today in regards to creating educational games is with children's attitudes about education. The attitude children have towards school, less interested and not actively involved in seeking solutions outside of the curriculum, tends to be the opposite of their attitude towards games. Prensky [40] elaborates that the attitude toward their games is "interested, competitive, cooperative, results-oriented, actively seeking information and solutions." Educational games need to have the same elements as pure entertainment games in order to maintain their fun factor. For educational video games to be successful, they should have meaningful, interactive and challenging worlds where players can progress at their own pace and learning curve [22]. Nielsen suggests that the three important factors for designing strong learning games are integration, motivation and focus. These are discussed in the sections below. 


\subsubsection{Integration}

The gameplay and the learning components should be tightly integrated such that when the player masters a game, he or she also masters the learning goals behind the game. Problems arise when games have little connection between the gameplay and what the player has to learn. A good example of this problem is demonstrated in Age of Empires II, a real-time strategy game that covers some of the important periods in history that are covered in the school curriculum. This game is not an educational game because historical knowledge is not needed to succeed in the game. Even though the historical vocabulary is mixed in with the gameplay, players just need to learn about the rock-paper-scissor dynamics in order to master the game. Since the learning goals are not integrated with the gameplay, many players will just ignore the historical information because there is no consequence for not learning [22].

An example where learning is tightly integrated with gameplay is TyperShark. This is just one of the many typing games where the main objective is to type the falling words. In TyperShark, the player must type the words that appear on the fish and sharks before they reach the scuba diver character on the screen. These typing games succeed because the skill the player is learning is integrated with the gameplay.

Therefore, a key factor for a successful learning game is the integration of the material to the learned and the game mechanics and play. The next factor is making sure that the game motivates the players and keeps their interest and engagement.

\subsubsection{Motivation}

Motivation is used effectively in educational games when it takes into account player psychology and what the player thinks is fun [17]. The game needs to be designed in a way that the player wants to accomplish the tasks set out in the game. According to Nielsen, motivation in games involves keeping the players engaged with well-balanced 
gameplay, a balanced reward system, quick feedback, and consequences that change according to the player's actions [22].

It is important to understand a player's psychology when discussing motivation. What makes a game motivating is determined by what a player finds pleasurable; this approach of looking at how a player thinks and feels when they play a game is called "psychographics" by Schell [44]. Schell explains that some psychographics are easy to understand; for example, for a player that is a "dog lover," creating a game about dogs would increase the chances of that player being interested or motivated to play the game. Other psychographics are not so concrete and can be traced to what people enjoy most, what they find pleasurable and desirable. Salen and Zimmerman [42] and Denis and Jouvelot [17] also mention that pleasures and desires are important factors that provide motivation to play a game. Denis and Jouvelot state that pleasures and desires are forms of intrinsic motivation and reflect the dynamics of a learning curve that requires constant adjustment to maintain the player's engagement. In their definition of pleasure, Salen and Zimmerman include "any physical, emotional, psychological, or ideological sensation" (p 330). Salen and Zimmerman also mention that the contrary factors (pain, frustration, and despair) are equally important for understanding the pleasures in a game.

Looking further into the different pleasures found in a game, Hunicke et al. describe eight pleasures that make a game fun [30]. Of interest to this thesis are sensation, challenge, discovery and expression. Sensation involves using your senses such as seeing an attractive visual or hearing pleasant sounds. Challenge is encountering a problem to be solved or the action of solving a puzzle. Discovery is finding something, whether it is a solution to a puzzle or an area or object in a game, and finally expression refers to the pleasure of expressing yourself or creating something.

Hunicke et al.'s list is just one enumeration of the various pleasures that exist in games and are factors in a player's motivation, which will be explored later. For 
instance, Schell mentions such pleasures as humor, possibility, anticipation, achievement, surprise and thrill. However, each player will place different values on the pleasures based on their preferences [7].

Schell mentions another pleasure concept, purification, that is relevant for this thesis. He states that "it feels good to make something clean" (p.112). Any game that has the player get rid of all the enemies or find all the missing objects takes advantage of the concept. A good example appears in Pac-Man, a popular arcade game from 1980, where the yellow pie shaped character has to eat all the dots in order to advance to the next level.

All of the pleasures are motivational elements, and a good learning game integrates these elements in some way or form. If a player is motivated to play the game and the game focuses on learning concepts then this results in a player interested and motivated to learn those concepts in the game setting. The "game" without motivation should be categorized as educational software instead of a game [22]. The last element Nielsen writes as a good educational game is the element of focus.

\subsubsection{Focus}

Nielsen describes focus as the game's emphasis on its learning experience. In a game with lots of historical terminology and facts, the player can focus on those facts by interacting and exploring a historical map or city. However, if the focus is on walking around and searching for objects in those boxes, then the player will only learn to walk around, search and identify boxes. This does not help the player learn the historical facts associated with that city. It is important to focus the main part of the gameplay on the learning activities.

Schell also states that focus is an important part of game design, but goes into further detail looking at the brain's ability to concentrate its attention selectively. Schell gives the example of a cocktail party setting; people have the ability to "tune 
in" to the conversation that they are participating in and "tune out" all the other conversations going on. This idea of focus relates directly to motivation and the desires of the player. The goal is to create experiences within the game that hold the player's focus as long as possible. To do this we must use motivation to capture the complete attention and imagination of the player, but keep the focus around the learning elements in the game.

Where in the World is Carmen San Diego is an educational game series that takes players to different geographical places around the world as Carmen attempts to arrest different villains. The games in the series are successful in teaching geography to its players by offering clues and information about the locations of the suspects. The player must learn about each location and deduce which location to go to, based on their clues. If a player chooses incorrectly, they are given clues that will direct them back to their previous locations. One of the factors that make the game interesting is the time aspect. The player has only a certain amount of time before the suspect "gets away." In that case, the player must start over and try to catch a new suspect. The time factor is important to make sure the player learns the correct information and makes the right choice.

Nielsen believes that these three factors of integration, motivation and focus are key to creating strong educational games. He also notes that the motivation factor is not as important when it comes to formal academic settings, where students are required to do certain activities. We have discussed that integration, motivation and focus are important; to help explain how to achieve these, we describe a concept called "flow."

\subsubsection{Flow}

The concept of having the right balance between challenge and progression is called flow. Salen and Zimmerman, Nielsen, and Schell all refer to psychologist Mihalyi 
Csikszentmihalyi's [13] definition of flow as "a feeling of complete and energized focus in an activity, with a high level of enjoyment and fulfillment." When this state of flow happens, the only thing we are thinking about is what we are doing and we often lose track of time.

Schell describes some of the key components that are necessary to put a player in state of flow:

1. Clear Goals: If the goals are clear, it is easy for a player to focus on completing them.

2. No Distractions: Distractions break flow by breaking the players' focus.

3. Direct Feedback: If the player has to wait for feedback then it is easy to get distracted.

4. Continuously Challenging: Challenge is a type of pleasure, but it must be achievable.

The gameplay must provide just enough challenge to keep a player in the flow state. If there is too much challenge, the player can experience frustration and too little results in boredom. Csikszentmihalyi describes this as a "flow channel." 


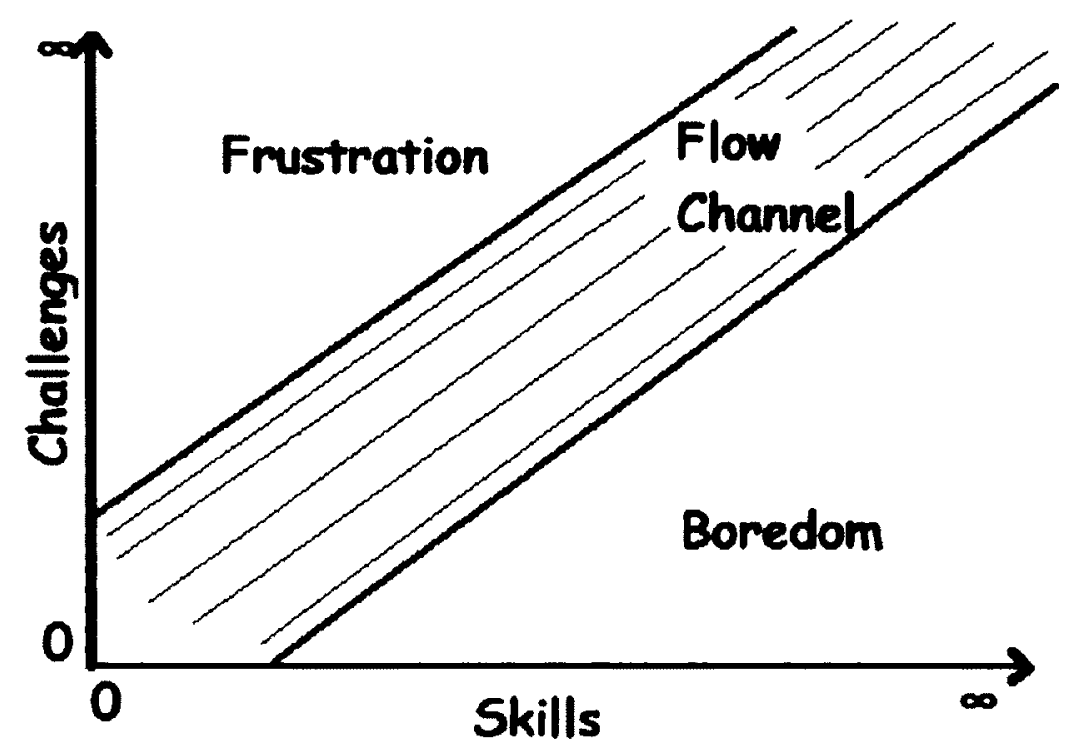

Figure 1: The flow channel (adapted from Csikszentmihalyi, 1997)

Figure 1 shows this flow channel and how there is a balance between the skills the player has and the challenge a game can provide. As the player's skill increases, the game must provide a similar increase in challenge in order to maintain that state of flow. Often, we see that game designers maintain this flow by increasing the difficulties of the level or the enemies as they get further into the game.

Schell notes that there is much debate about whether it is good to have the flow interrupted when a player gets to a level or an enemy that they cannot easily defeat. The player ends up spending more time being frustrated and may give up on the game. This scenario can happen but the argument remains as to whether some level of frustration is a good thing or a bad thing. It is bad because the player will get frustrated and sometimes quit and never return. On the other hand, you could argue that, although such a situation digresses from the state of flow, it ensures that the player spends more time perfecting their skill or learning the material.

When players persist and overcome the obstacle, they feel a sense of accomplishment. As we mentioned before, a player's focus relates directly to motivation and 
psyche, and a sense of accomplishment is a strong motivating emotion. It is also one of the more important pleasures that can be used to create successful educational games.

\subsubsection{Achievements}

Richard Bartle [7], in his taxonomy of player categories, includes achievers; he argues that the main goal of this type of player is to gather points, get higher scores, and rise in character levels. Bartle is referring to achievers in a particular class of online roleplaying games; however, Schell describes a similar idea of achievement, or completing extra goals that may not be part of the main gameplay, as another pleasure that players enjoy. One way that game designers tap into this particular pleasure is to make it possible for players to complete a game at multiple difficulties; in order to complete the achievement the player must spend enough time to play through the game on each of the required difficulty settings [36].

Another way of creating a sense of accomplishment is with trophies or medals awarded when the player has completed a certain task, regardless of how it relates to the main gameplay. Many of today's popular gaming systems, such as the Xbox 360 and the Playstation 3, use achievements as a universal reward system for their specific console. Mikael Jakobsson explains how it works for the Xbox 360 gaming system [31]. "Each game must have 1000 points that will be divided into a maximum of 50 achievements. When a player gets points and achievements, they can share and compare their points and achievements with others." Salen and Zimmerman refer to this type of achievement system as a part of the larger social context of a game. Achievement systems provide a sense of community and competitiveness among players.

Although the concept of achievement systems is garnering much attention because of the popularity of the gaming systems, it is not a new concept. Jakobsson explained 
how Atari, a video game company, used a similar system 30 years ago that worked in a more manual fashion. Players had to take pictures of their television screen if they achieved a certain score and mail the photos to the developers. In return, they received a physical badge so that they could show it off to their friends.

\subsubsection{Game Mechanics}

We have covered how factors such as integration, motivation and focus are important when designing games; now we will take a look at game mechanics and how they make up gameplay. Schell defines game mechanics as "the interactions and relationships that remain when all of the aesthetics, technology, and story are stripped away." $\mathrm{He}$ notes that there is not one taxonomy of game mechanics that can cover everything due to the complexities of gameplay. There are a couple of game mechanics that are important for this thesis.

Prospecting is a game mechanic that involves searching, scouting or scanning for something within the game. It relates directly to the pleasure of discovery, one of the pleasures identified by Hunicke et al., listed earlier in this chapter. A good example of this is found in Minesweeper [34], a game where the player has to flag all of the hidden mines without hitting any of them. A player can click or prospect one of the squares to reveal some valuable information about the potential mines around that square. However, they also run the risk of hitting a mine and therefore losing the game. This risk is part of the fun as it falls under Schell's chance category. 


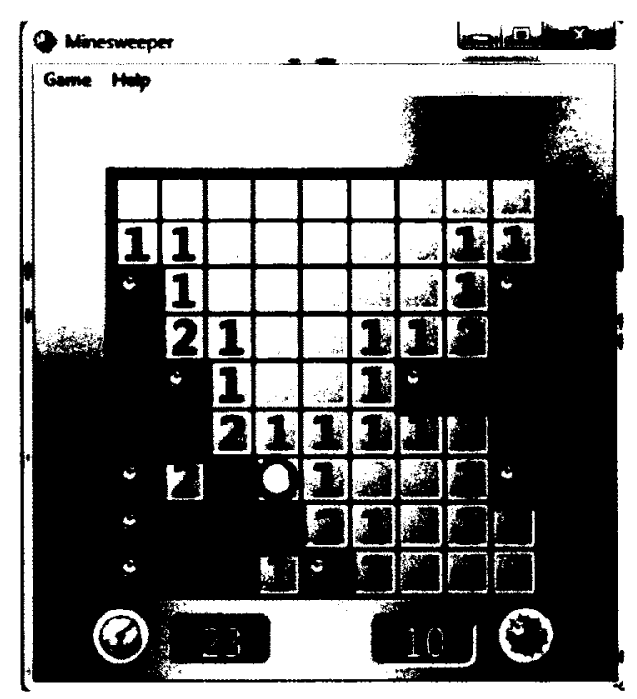

Figure 2: A screenshot of the Minesweeper game

In other games, prospecting does not have as big of a risk as Minesweeper does. An example of this is in Mass Effect 2 [8]. Players can scan planets for resources that are valuable for accomplishing certain tasks in the game (such as upgrading weapons), and they can also discover other missions to accomplish. To scan a planet, players click on a planet, then rotate it as an in-game scanner displays a grid about the resources in the area. When players decide on an appropriate area, they can launch a probe to harvest the resources.

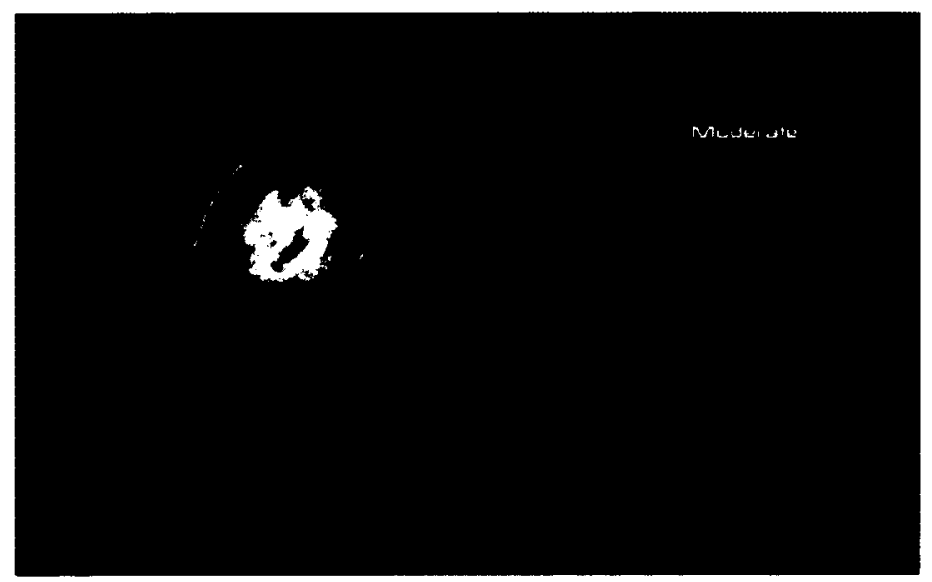

Figure 3: A screenshot of the Mass Effect 2 scanning mechanic 
In both examples, the player has incentives to use the prospecting mechanic to gain some type of reward, whether it be information or a resource in the game. The prospecting mechanic is a way to engage the player by using the element of chance and surprise along with mystery and possible failure. Prospecting also helps the player focus on particular areas in the games space, but a better way to engage a spatial sense is with sketching.

Sketching is a game mechanic that enables the player to express themselves in a natural way. It helps the player express their intent in relation to spatial concepts [49]. This mechanic satisfies Hunicke et al.'s pleasures of sensation and expression. While some games use a recognition-based approach for the sketching interface [26], others apply a visual and conceptual understanding within the game to interpret the input from the sketcher [19]. A good example is the sketch-based war strategy game called nuWar, created by Dunham et al., that features sketching. In this turn-based twoplayer game, each player sketches war tactics on the screen to describe strategies to their respective teams. When both players are ready, the game shows the results of their actions by simulating a set amount of game-time. In Figure 4, the Red-side player sketches the course of action, in this case an ambush. 


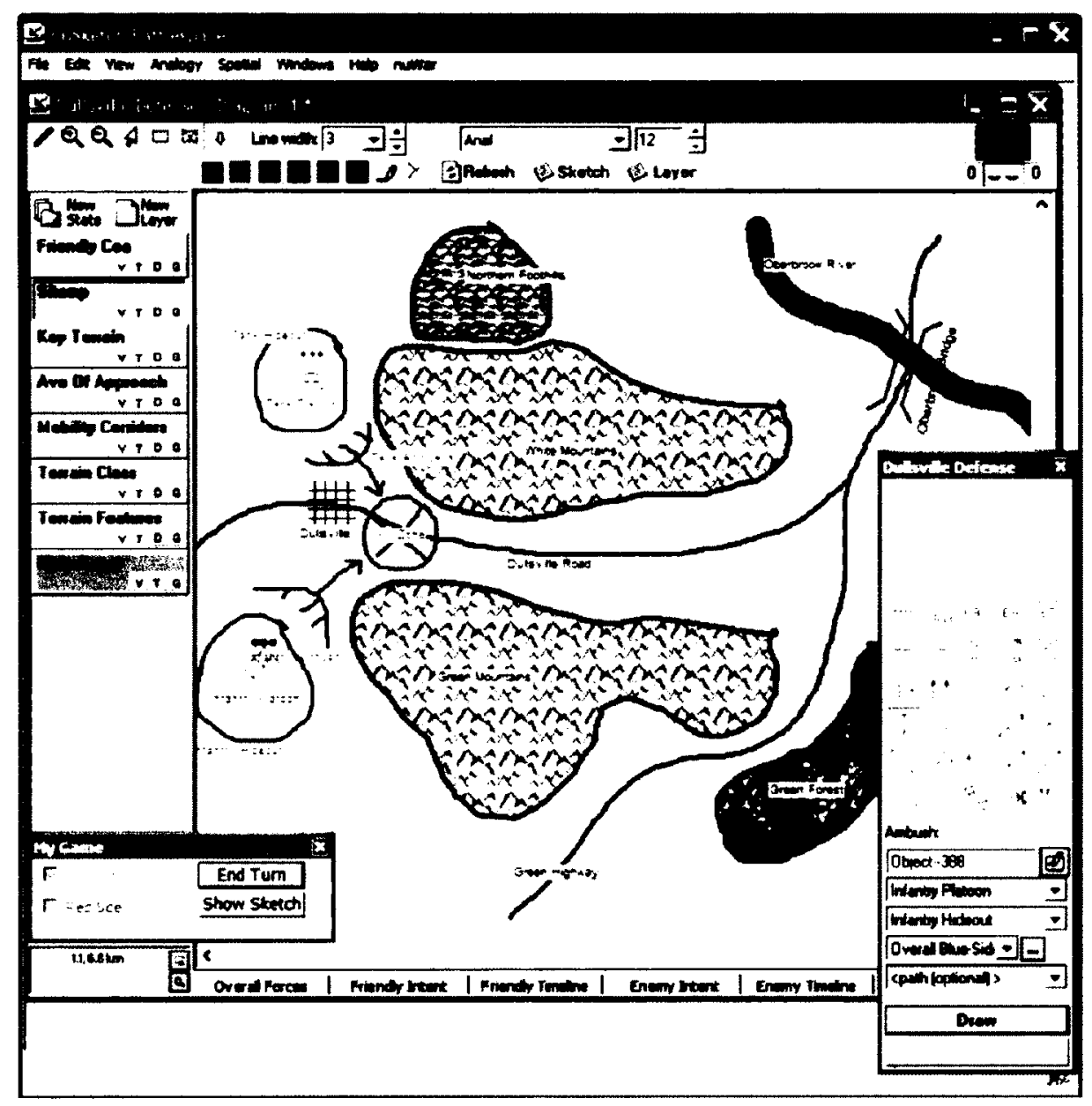

Figure 4: A screenshot of the game nuWar that uses a sketch mechanic and shows the player describing an ambush

Prospecting and sketching are two mechanics that get players to focus and pay attention to specific areas in the image. Two game mechanics that were also considered were spot-the-differences and password-type. In the spot-the-differences mechanic, players try to find a set number of differences between two very similar images. One image is most likely derived from the other using some image or photo manipulation tool that changes small, specific details, such as a break in a line or a missing eye. The player must concentrate and pay attention to the details in order to notice differences and identify them. The creator of the game content would need to make sure that the differences between the images involve the significant elements of the images to make 
sure the player would pay attention to them; however, typically differences in these games are very minor and we wondered if players would remember the significant details. We envisaged technical problems with removing features from the images. Even if we managed to overcome the technical difficulties, other people might have trouble doing it and the framework would then not scale well.

In a password-type game, the players would be responsible for creating and solving the password-type puzzles. To solve one of the puzzles, a player would be shown parts of an image (the rest would be blurred or blacked out) and try to guess the name of the image or the significant details that represent the password. In order to create these image puzzles, players require a certain level of understanding and knowledge of the image to decide which areas to show and which to blur out. They also have to decide the password key, name of the image, name of the artist, or the specific detail required in order for players to solve the puzzle. This mechanic would get the player to study the images while creating the puzzles and would test their knowledge when solving puzzles they did not create. One problem with this is that we cannot embed facts or other learning material easily in the game. The players can study the image, but not the history or symbolism within the game environment. Another problem is that we need a community to play and that it would not be easy to test players individually. In addition, we would have had to spend a lot more time with this mechanic to set up infrastructure before getting to the point of being able to test it. Also, since players would be creating the content, we would need some sort of solution to check for puzzles that are incorrect or did not make sense.

Both the spot-the-differences and password-type mechanics seem good for testing a player's knowledge; however, they do not seem good for helping students learn the material initially. The spot-the-differences mechanic only allows for identifying the differences, which may or may not be significant in the image. In the passwordtype mechanic, previous knowledge of the image is required for solving the puzzle. 
Essentially, the only beneficial type of learning lies in creating the puzzle; however, as we noted before, the players would be able to study the images and not information about the history. While both of these game mechanics have merit, they both have a limited variety of activity and it was decided to proceed with Sketch and Learn because it allows for a greater scope for continued interaction with the image.

\subsection{Visual Attention and Memory}

We have covered game mechanics and discussed the role they play in educational games and how important it is to understand human psychology in designing those mechanics. When designing an educational game with any type of visual element, understanding how to get the player to perceive what you want him to perceive and remember what you want him to remember is necessary to designing a good learning game.

What a player sees and remembers in a game is determined by how much attention is paid to the subject. Visual attention allows players to select the information that is most relevant to their ongoing behaviour. Chun and Wolfe [12] explain that visual attention is relevant in any situation such as driving a car or walking down a street. In order to drive or walk safely, people need to be able to detect and interpret signs. The reason the signs are in certain locations, shapes, sizes, colours and languages are to get the attention of the person so that it is noticed, but not too distracting to cause the driver to lose focus.

In researching spatial and temporal attention, Chun and Wolfe considered the theoretical, behavioural and neurophysiological work done in the area. Of their analysis, the more important points for designing games is how the player decides what to focus on and how memory works with attention. 
First of all we have to focus on attention. The brain has a remarkable ability to cope with a vast amount of information and has some attentional mechanisms to handle overload. The attentional mechanisms have two main roles: to select behaviorally relevant information and to ignore irrelevant information. The brain processes all the information and selects what it wants to focus on, which is called active selection. Although many studies have focused on active selection [38] [9] [18], of interest to game design is attentional selection over space and time. Typical studies have subjects focus their attention on part of a spatial array and report on the focus of their attention [23]. Chun and Wolfe describe this type of attentional spotlight as "the feeling that attention can be deployed, like a beam of mental light, to reveal what was hidden in the world."

There have been important experiments for understanding spatial attention, from cueing experiments that have been used to study spatial attention as a spotlight to overlapping movies that looked at object-based attention. This has been discussed by both Posner and Cohen [39], and Neisser and Becklen [37]. Posner's cueing experiment had subjects fixate on a central box at the beginning of the trial. Then, there was a variable target delay until the cue lit up, at which time the subject had to press a button as quickly as possible.

According to Chun and Wolfe, the cues aided the detection of the target and also helped the subject respond to the stimuli presented at the cued location. Chun and Wolfe also noted that the overall perceptual load of a particular task has to be high enough to ensure the subject would not be able to process the targets that were not part of the event. If the perceptual load was not high enough, targets would be able to process information and objects that were not part of the target. A way to increase the perceptual load in a game is by adding visual effects.

An experiment by Neisser and Becklen tested whether attention is based on space instead of time. Their experiment showed two separate movie clips that overlapped 
each other in space. One clip showed people throwing a ball and the other clip had people playing a hand game. The subjects were to focus on one of the overlapping clips and respond to certain events in them. Even though the clips overlapped each other, it was rare for the subjects to respond to the odd events in the unattended movie. The experiment showed us that selection is based on the objects and events over time, and not just based on space. However, in terms of the overall visual attention, location does play a critical role [11]. In conclusion, a game designer should be careful in placing critical visuals that appear at the same time on the screen too far apart, since a player would only be able to pay attention to one of them.

The review above describes some of the behavioural mechanisms of attention, but another important factor to consider when designing educational games is how a player will remember the important details they see on a screen. Attention and the processing visual and spatial information invokes a system of working memory, which psychology textbook author James Nairne called the "visuospatial sketchpad" [35]. Nairne gives an example involving the visuospatial sketchpad where a person would try to count the number of windows in their house by visualizing themselves going through the house. This helps with memory as people associate the windows with real physical locations.

The spatial memory concept can be transferred to other situations, such as when a person goes through a virtual environment, they will be able to remember the locations of certain items or objects, or when a person is asked to recall a specific feature in an image, he will visualize the location of that feature. Similarly, colouring books such as The Biology Coloring Book [28] are used in schools as a study aid. Since the students spend time with an image and colour the specific elements, they remember where those elements are located within that image. There many books like it, covering topics from anatomy to geography and even botany. 
Spatial memory is important to educational games. Game designers should design the game with the knowledge that players will associate objects with their locations on images or within virtual environments. Spatial memory combines with visual attention to help the player learn through the game.

\subsection{Conclusion}

We began with a look at how art history is taught and argued that interactive technology could help students learn to recognize and analyze significant features and facts about works of art, a key skill in this field of study. In addition we found that sketching is considered an effective technique. We then examined the various elements that need to be considered when designing an educational art history game.

A good educational game features tightly integrated gameplay, continually motivates the player throughout the game, and keeps the player focused on the game. These elements put together along with a fine balance between challenge and diffculty keep the player engaged in the flow of the game. Achievements and the various game mechanics can be used to extend gameplay and motivate the player to have fun while learning. Of particular interest to educational art history games are the sketching and prospecting mechanics.

Since focus and memory are important to an educational game, we examined some key concepts in visual attention and memory. Highlighted features without overloading players with too much information helps them focus on those features and remember them better. In addition, players will remember the locations of those features, a fact that game designers must take into account. 


\section{Chapter 3}

\section{The Design of Sketch and Learn}

Sketch and Learn is specifically designed for the creation of sketch-based games to help players learn a domain where images are prevalent. As we discussed in the Theoretical Framework, prospecting and sketching are the key game mechanics to help players remember relational details and keep them motivated to learn.

In this chapter we examine the important elements of the design of Sketch and Learn: controllers, player hub, and game view. In particular, we describe the overall framework, the elements of game mechanics we used to get players to spend more time on and learn more about the works of art, and how we implemented those mechanics. We explain how prospecting and sketching promote learning and how they work with other mechanics such as purification to get the player to spend more time on the image. Screenshots are presented to help illustrate and complement the descriptions and experiences using Sketch and Learn. Mechanics explored in the Theoretical Framework that were found to be outside of our scope are discussed as well as the limitations of this particular design. The last section in the chapter covers the pros and cons of using text to deliver the facts about the images as opposed to audio or video. 


\subsection{Controllers}

Sketch and Learn was created using the ASP.NET MVC 3 framework which is used for creating scalable web-based applications. The framework enforces the Model View Controller pattern so that we isolate the domain logic in the controllers. In our implementation, we designed controllers to add and delete artists' information, manage users, create content, and handle the game, as follows:

\section{Artist}

The artist controller lets users list the artists and view biographical information and the images created by each. This controller also allows users with specific permissions to add and delete artists.

\section{User}

The user controller allows the user to create an account, and login and logout of the application. It handles passwords and keeps track of whether a user can create content or not.

\section{Content}

A controller to create content was implemented so that it would be easy to create content for the game. It allows users to add or delete artists, images, glyphs, and detailed information about the images. This information gets written to the database so that the game controller has access to it.

\section{Game}

We designed the game controller to handle all of the actions that take place in the game. It retrieves the images from the database as well as the player's progress on each image and displays the images and the information in the Player Hub; see section 3.2. Then, if a user clicks on an image, the controller brings up the Game View (see 3.3) for that particular image and displays the 
level of completion by the user. To do this, the controller gets the image, artist name, details and all the glyphs belonging to that image, and sends them to the user's Game View page.

These four controllers proved adequate to ensure a smoothly functioning game. Additional controllers could be added to this model, for instance, a controller to display images based on when they were created.

\subsection{Player Hub}

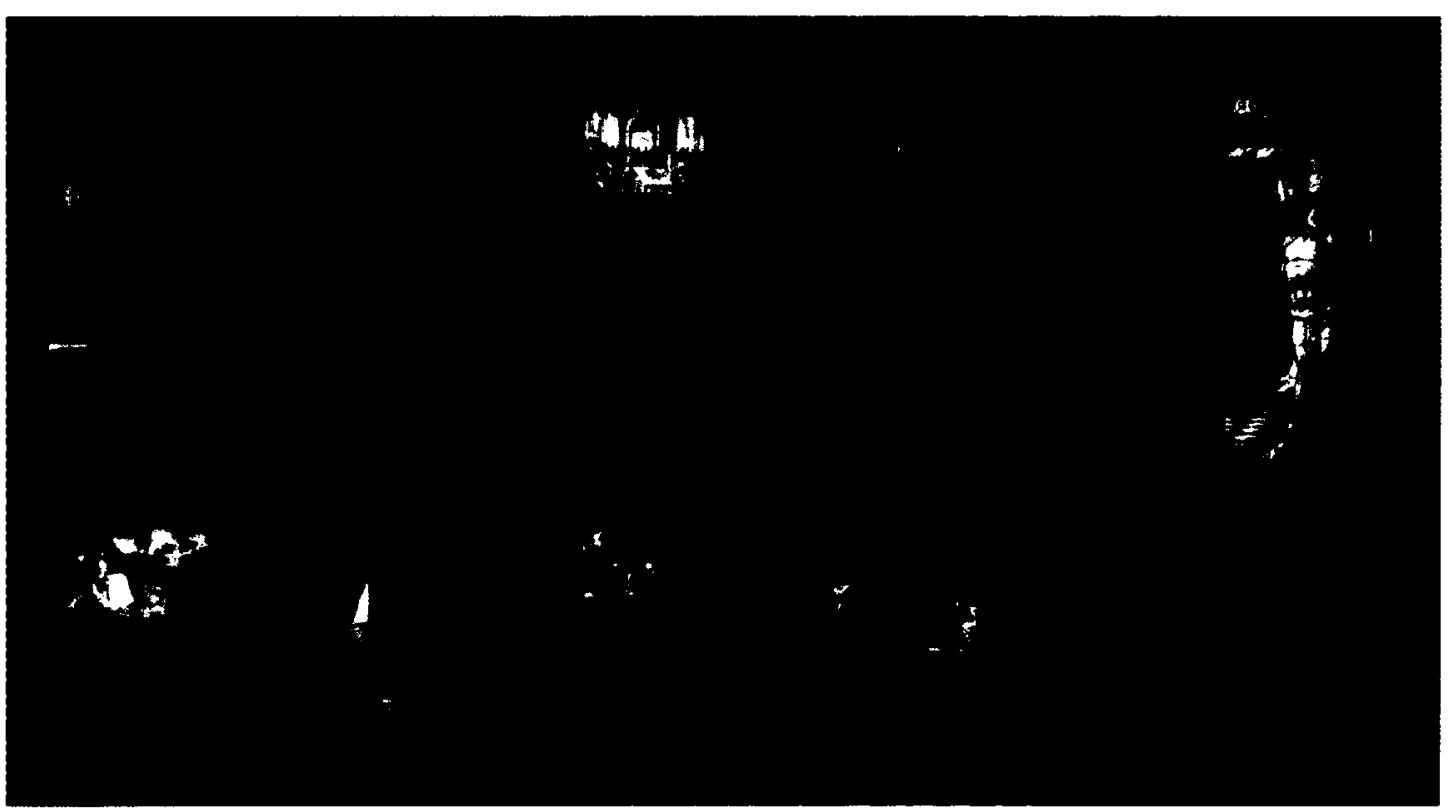

Figure 5: A screenshot of the players hub

The Player Hub is the front page to Sketch and Learn and the page players use to access images. The player can see all or a subset of the images available to learn. The images are shown in a smaller size along with the name and the player's progress. The smaller images allow for the display of a larger number of images on the screen. The images are presented on the screen across and then down with a maximum of 12 per page, with a link to the other pages if necessary. The progress is shown 
either as "Image not yet viewed" or the number of glyphs found compared to the total number to be discovered. We show progress to help players know if they have partially or fully completed an image, allowing them to have a sense of achievement as they get closer to their goal of learning the whole set of images [44]. It also helps the player to choose which image to work on.

\subsection{Game View}

The Game View contains the image that the player is focusing on, the title and name of the artist, the reward area, and the information area (Figure 6). The player interacts with the image by prospecting and sketching the glyphs. A glyph is an outline, a drawing, a shape or an area that the player must sketch in order to obtain valuable information about the image. In Figure 7 below, the green outline is a glyph. The player can also click on the artist's name to be taken to the artist's page.

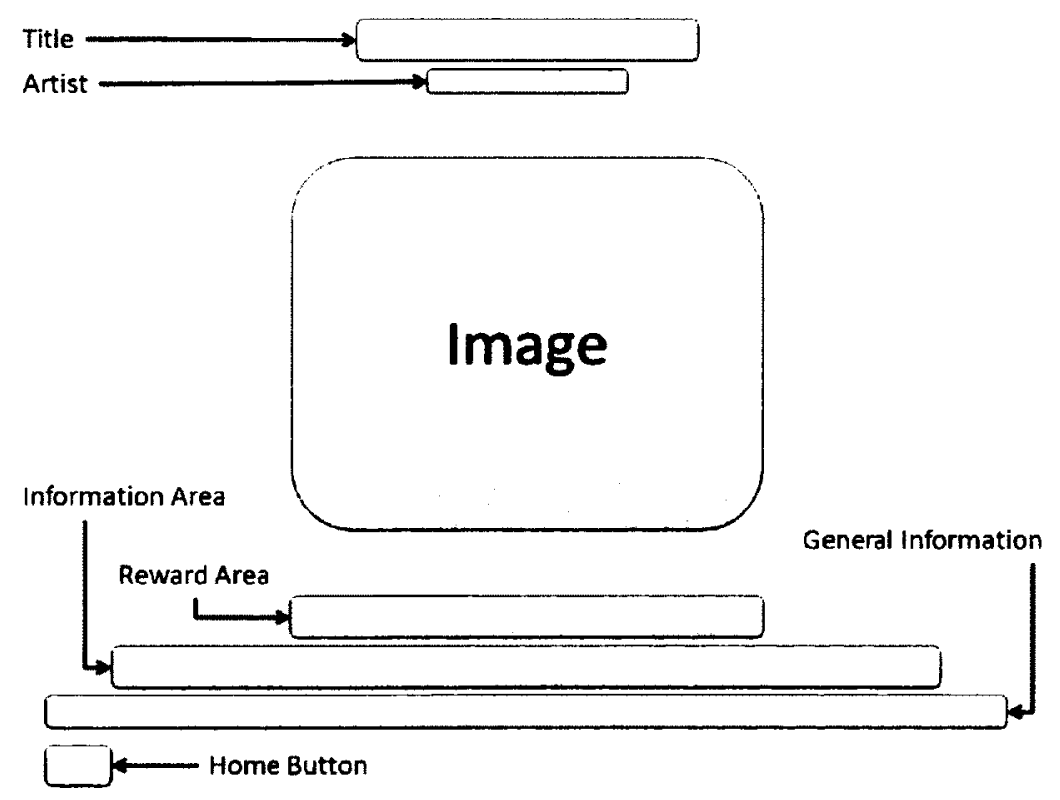

Figure 6: A schematic of the Sketch and Learn game page 


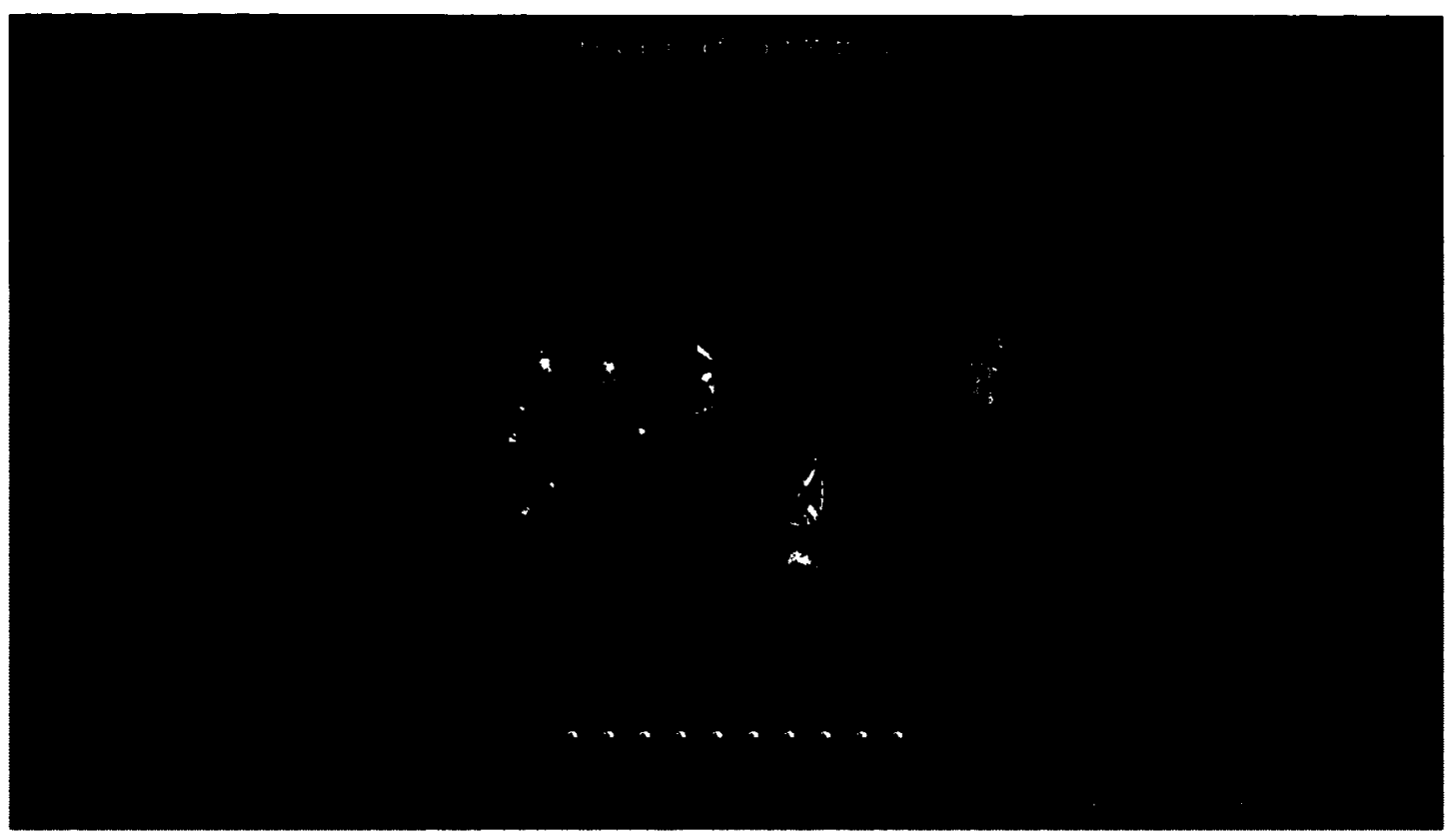

Figure 7: A screenshot of the full Sketch and Learn game page

As the glyphs are completed, star icons light up in the reward area below the image and the significant fact associated with that glyph appears in the information area. Under the reward area (star icons) is the information area, where the text for each glyph appears as well as a general description of the image with its location and date of creation. Finally, a home icon at the bottom left corner of the page, there is a home icon, allowing the player to return to the Player Hub.

We developed the Game View using HTML, Javascript and CSS on top of the ASP.NET MVC 3 framework. We also used a couple of open source tools, Processing.js and JQuery, to create the interactive game mechanics, which are outlined in the following sections. 


\subsubsection{Prospecting}

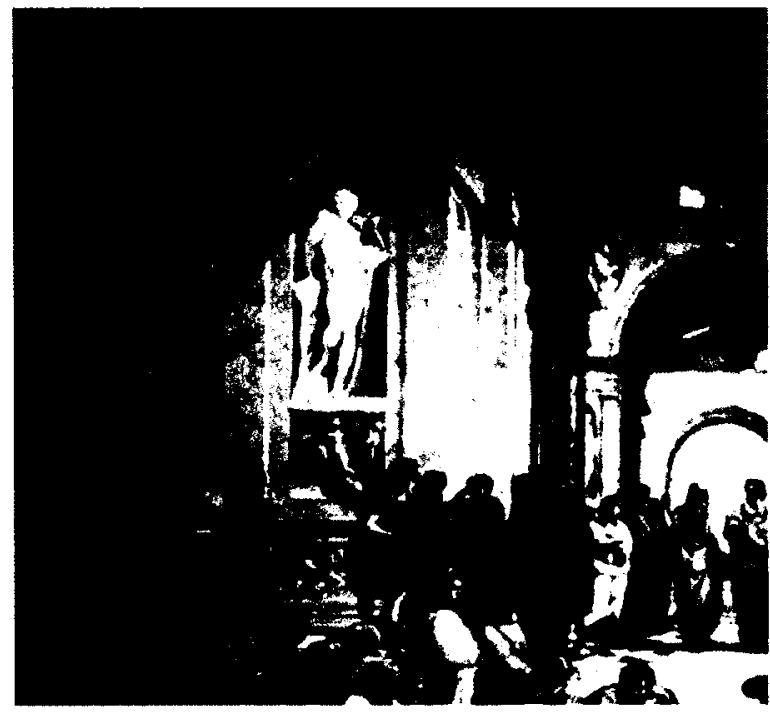

(a) Prospecting area

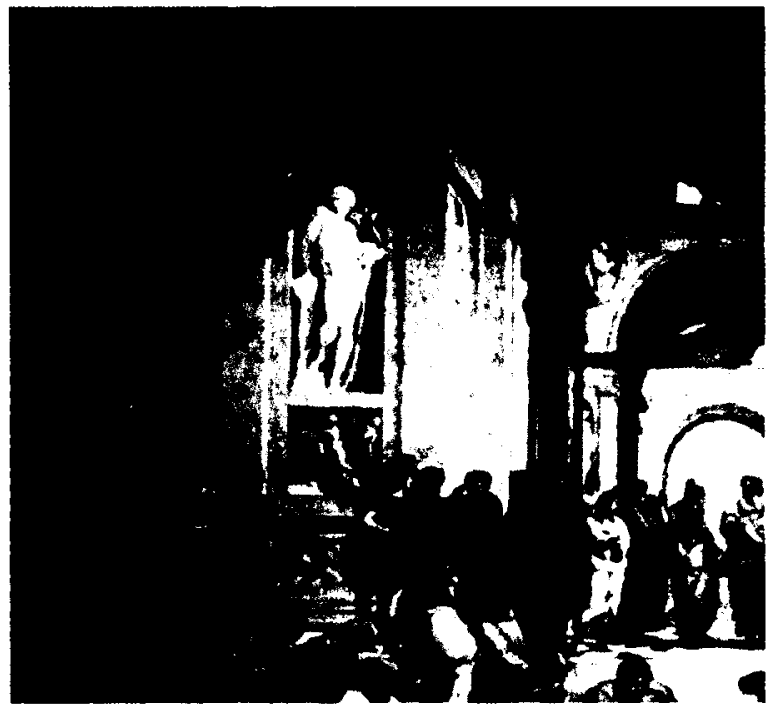

(b) Prospecting area shrinks

Figure 8: Screenshots of the prospecting mechanic

In order to find the glyphs within the image, the player must locate an interesting feature in the image and click on it to see if there is a glyph. This is called prospecting and is shown in Figure 8 above.

In the figure, the player clicked near the top left of the image. A semi-translucent circle appears, showing the player the area that was searched. Once the player has prospected, there is a five-second delay until the player can search again. The circle slowly shrinks as the time counts down to help the player know when he can start prospecting again (see Figure 8b). If the player tries to prospect another area before that time period is up, nothing will happen.

When the player prospects an area and a glyph is within the search radius of the circle, sparkles appear at the start of the hidden glyph. They fade away shortly after. By discovering a glyph, the player has unlocked it and now has the ability to see 
the glyph when the cursor is close, sketch it, and reveal the information about that element of the image (see Figure 9).

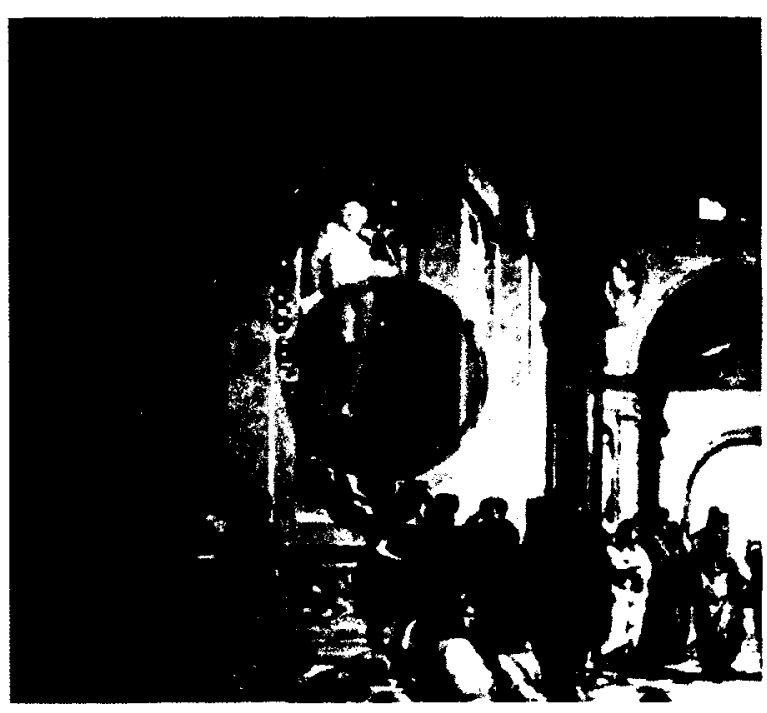

(a) Prospecting discovery

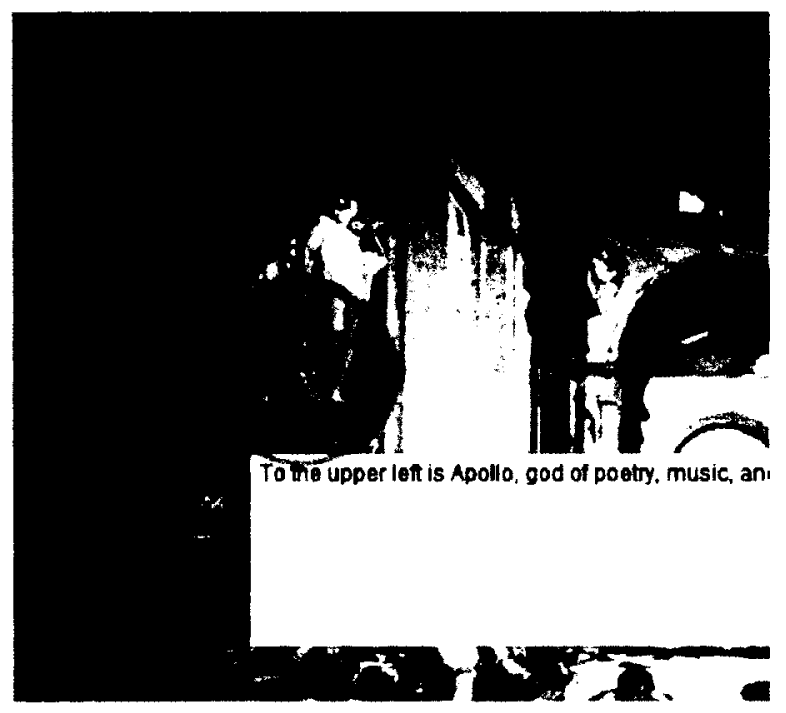

(b) Sparkles a start of glyph

Figure 9: Prospecting and discovering a glyph

The prospecting element motivates the player to scan and observe all of the details in the image. The challenge of prospecting is rewarded by finding a glyph linked to a significant element of the image and learning about that feature. The player keeps looking for the pleasure of discovery and the visual pleasure of the sparkles, which makes prospecting fun.

Prospecting also helps the player remember details of the image even if there is no glyph associated with that particular spot on the image because he or she needs to scan the whole image. Players will look in the corners, look for different shades or changes in colours, interesting objects, and other elements of images that would go unnoticed when quickly glancing at the image.

The delay between mouse clicks was designed to prevent the player from rapidly clicking in an attempt to find the glyphs more quickly. The goal is to make players take more care in their investigation of the image. This delay gives each prospect a type 
of cost, in terms of time, that the player will have to consider before every prospect. However, if the delay was too long, players might get distracted or lose interest. Although the choice of five seconds was arbitrary, our limited testing showed that five seconds provided enough of a challenge without frustrating the player, maintaining flow. This could be an interesting area for further research.

\subsubsection{Glyphs}

Glyphs play an important role in the Sketch and Learn as they are the main element that players must discover and then trace. Three main types of glyphs can appear in the Sketch and Learn, associated with an object, an element of design, and a general concept for the image. See Figure 9 for examples of the different types of glyphs that can be discovered in the game.

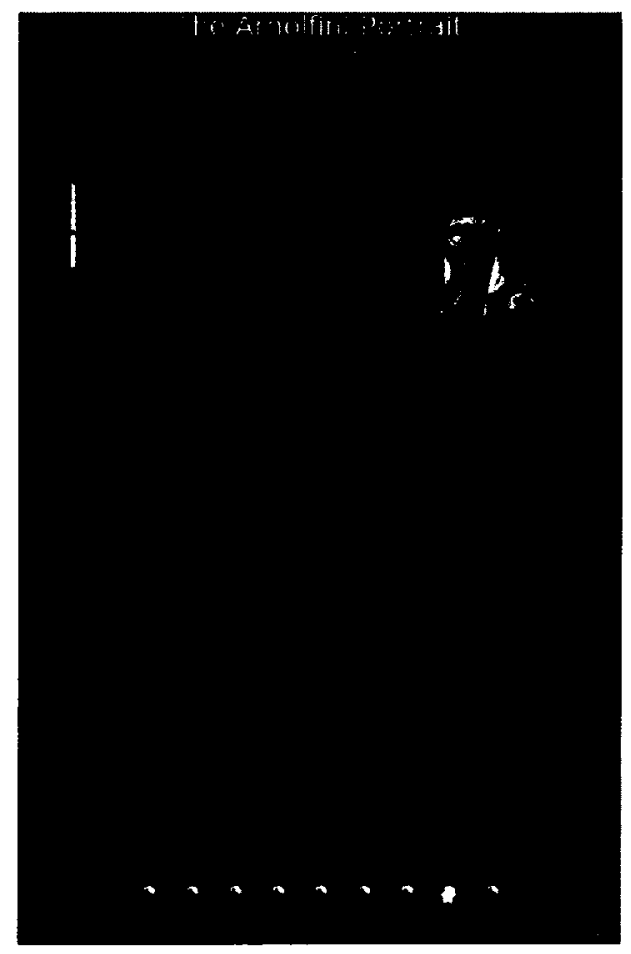

(a) Glyph 1 


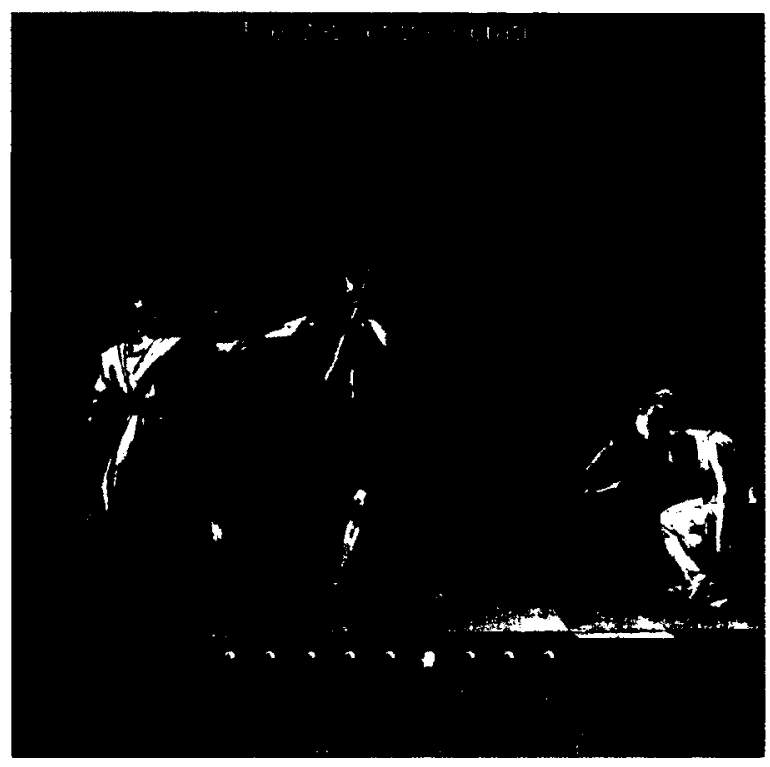

(b) Glyph 2

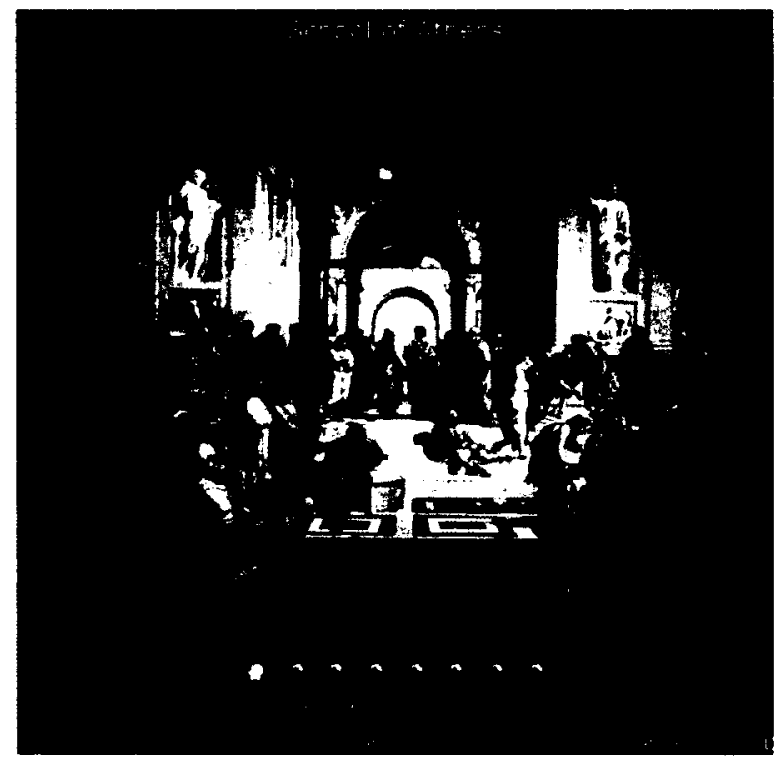

(c) Glyph 3

Figure 9: Types of glyphs

Figure 10a shows the outline of a dog and the fact that accompanies it describes the dog to be a symbol of fidelity. This type of glyph clearly outlines the object in the picture and has a description for the exact object it outlines. Figure $9 \mathrm{~b}$ shows a glyph that outlines a portion of the Roman arch, which is an element of design to connect the three groups of images. This type of glyph has a description that explains how the design element is used in the work of art. The last glyph, Figure 9c, outlines an element that by itself is not significant. It is connected with general information about the work of art as a whole: in this case, the fact that the work of art is typical of the artist's style.

The creator of the content is not restricted to creating these types of glyphs and has the flexibility to provide information ranging from very specific details to general facts about the image. Now that we have described the different types of glyphs that a player can find in the game, we will present the sketching mechanic. 


\subsubsection{Sketching}

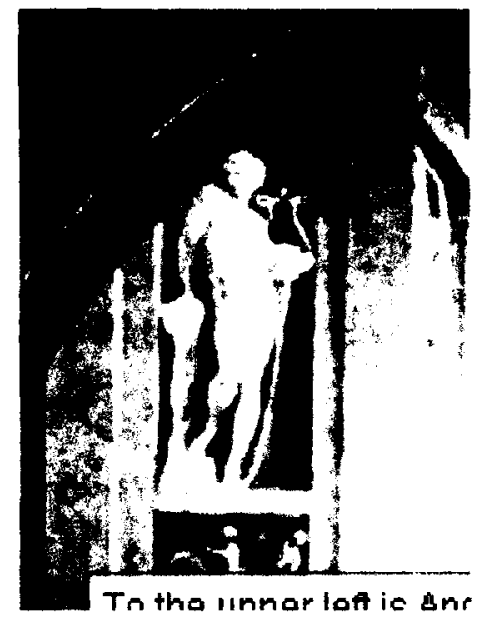

(d) Starting the sketching

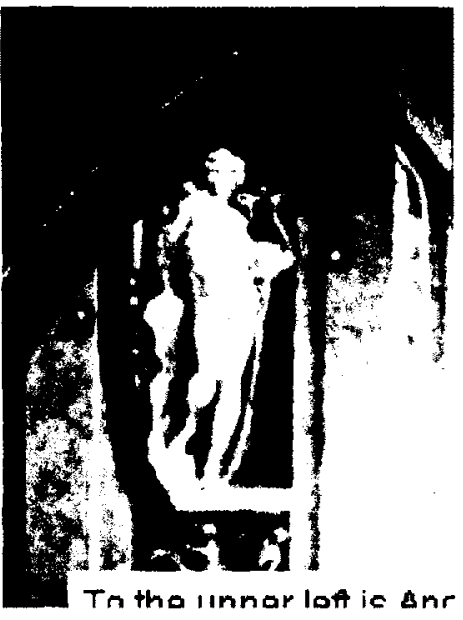

(e) Sketching part way

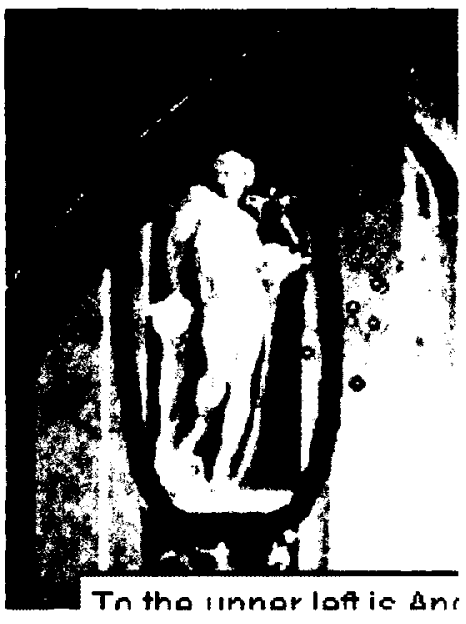

(f) Sketching complete

Figure 10: Sketching a glyph

In order to encourage the player to focus on the area where the glyph is located and to pay attention to the information associated with it, we designed a sketching mechanic. Our sketching mechanic emphasizes the area of interest by getting the player to sketch the outline of the glyph and when the player successfully traces the whole glyph, a sound notifies the player and the glyph slowly fades away and sparkles appear rewarding the player with a "ta-da" effect.

There were a few important decisions we made when designing the sketch mechanic. After the player prospected the glyph, we still wanted the user to focus on that area, so we made the glyph hidden until the player moved the cursor close to the glyph. Hiding the glyph, even when they have prospected it, and only showing it when the cursor is close, encourages the player to remember where the glyph is located.

To make the sketching guide stand out, we designed it to show the outline of the glyph in a bright green colour with a thin black outline. This contrast of light and dark allows the glyph to be seen clearly on any image. To keep the player challenged 
and engaged throughout the sketching process, we decided not to display the whole glyph at once. As the player sketches, we only show a small part of unsketched portion. We also decided that the portion of the glyph that has not been sketched should be a different colour. We chose this colour to be red with a black outline because it highly contrasts the green and is distinct on any image. The unsketched red shows the player the path to sketch. Figure 10 shows the three versions of one image with the glype at different stages of sketching: starting the sketch, partially completing the sketch, and completing the sketch.

Another decision that had to made related to challenging the player. The following conditions caused the player to go back to the beginning to restart the sketch:

- the player moves the cursor too far away from the glyph

- the player moves the cursor too fast

- the player moves the cursor too slowly

This made the player focus on what they were doing and provided some degree of difficulty. Testing showed that the glyph could not be too complicated to trace or the player became frustrated. We had to work on the sensitivity to the positioning of the cursor and use glyphs that had smooth curves and few corners.

To help reinforce the information connected with the glyph, a textbox appears with the associated information as the player traces the glyph. In Figure 10, the text provides information about the statue that is being circled.

To give emphasis to the information in the textbox and allow the player some time to read it, we decided to leave it on the image for a short period of time. Testing also suggested that a period of five seconds proved sufficient for most players to read the blocks of text. However, we did allow players who read quickly to click the mouse and close the box before continuing prospecting for other glyphs. The text is still 
available for review in the information block of the Game View, below the image (see section 3.3.4 below).

This tracing or sketching helps the player's memory and also mimics drawing or sketching with a pencil, which, as discussed previously, helps the player learn and remember the image. The visual effects aid in fixing the information with the specific location which is a key element in memory.

\subsubsection{Stars}

In order to provide the ability to review the glyphs and images, we added a set of star icons that represent each glyph. If the icon is grey, it means that the glyph is not yet complete. When the glyph does get completed, the star icon changes to a gold colour and a positive sound gets played. In our case it is the Super Mario Bros coin sound from the classic video game that many players would associate with video game rewards.

Figure 11 shows one star lit up, because a player has put the cursor on top of it. This causes the associated glyph to appear in green and displays the related text in the information area. Players can select any gold star to review the information and keep track of where the glyphs are located.

The stars also function as a way for a player to track his progress, providing a visual goal in terms of the amount of remaining glyphs to find. By showing the player how many glyph remain uncompleted, Sketch and Learn takes advantage of the pleasure of purification discussed in the Theoretical Framework. Players will feel the need to discover and sketch all of the glyphs in the image until there are none left, giving it a clean feeling. 


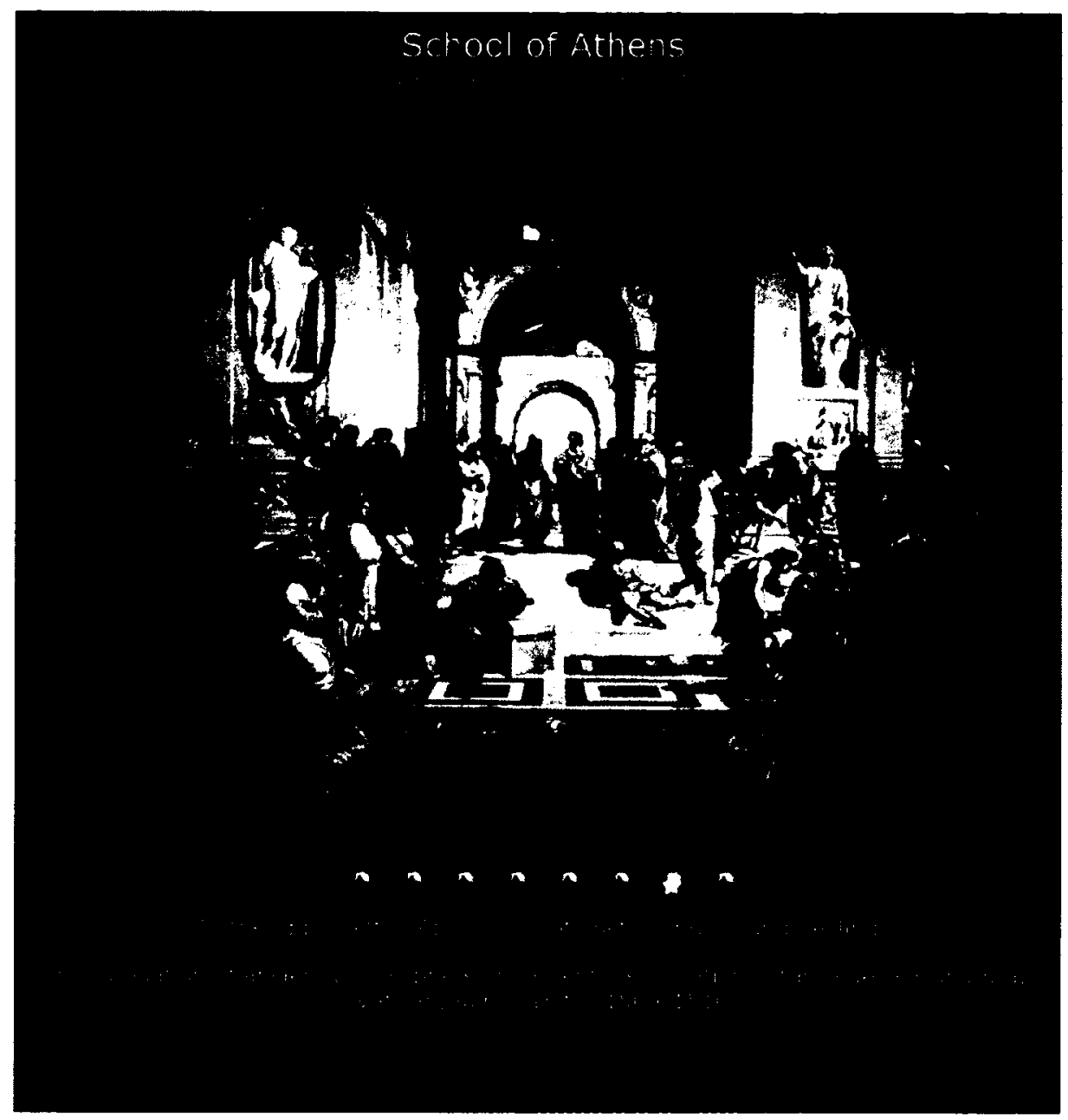

Figure 11: A screenshot of a complete glyph

\subsubsection{Fact Delivery}

One of the important decisions we had to make was how to deliver the information and facts to the player. The facts could have been embedded in the form of audio or video, but we decided text was the best delivery method for Sketch and Learn. This section describes some of the advantages and disadvantages of using each method and why we chose text to deliver the facts.

Our first idea for information delivery was to use audio to deliver the facts to the players. The player would be sketching parts of the image and simultaneously listening to audio facts that accompany the glyphs. The advantage of using audio to 
deliver the facts is that it does not take up any space on the screen, so the player would see the image at all times. This would be great if it were not for the disadvantages. Creating the audio content, getting an actor to read and voice the appropriate details, is costly and time consuming. The alternative of computer generated, synthetic, voice just won't be appealing to the player as it sounds fake and, making it difficult to understand [16]. The other disadvantage is that the players would have to listen to the information at the speed that it is delivered. However, if we presented the facts in text form, then the player could consume the knowledge at their own pace.

Since audio facts are difficult and costly to create, we decided to use text. The advantages in the delivery of facts using text lie in the ability for the designer to create content quickly and for the player to learn them quickly and to view them whenever they want. As mentioned before, having facts in text allows players to read them at their own pace. Creating text content is much simpler than creating audio content, especially since a lot of content is already available in textbooks and on the web. This also helps us compare the Sketch and Learn style more directly to a textbook style as both use text as the medium to deliver content. A disadvantage is that text content needs to be displayed on the screen. This will break the players focus from the image as the player must concentrate on the text.

The last idea that we considered using was video. Video is a great learning medium and is widely used in schools and classrooms around the world. The problem of using video for our solution is that it has all of the problems of audio and text combined. Creating educational videos is even more costly than creating audio. We would need actors, editors, digital graphics, etc. This would make it very difficult to create large amounts of content. Another problem with video is that it interrupts the player's flow of observing the image and forces the players to view the video in its entirety to learn everything about it. We decided this would not be desirable. 


\subsection{Limitations}

We are aware that the design could be improved by the inclusion of some game mechanics. Certain game mechanics would have required a more long-term time frame in order to test the results, for instance, an achievement system and different levels of images to complete, both of which would be supportive to learning the images.

Adding an achievement system to the framework would increase the players' motivation to learn. It would do this by promoting and positively reinforcing performing certain tasks in the game. There would be trophies for small tasks such as sketching their first glyph, completing their first image, and learning five images from a particular artist, to larger tasks such as completing 100 images from a certain time period, completing all images from a certain artist and even completing all images available. All of these side tasks provide clear goals that encourage players to play Sketch and Learn and learn the images. Achievement systems, as discussed in the Theoretical Framework, have been tested thoroughly and proven effective in games so we figured that we did not need to test this idea.

Another idea similar to an achievement system is having different levels of completeness for images. For each image in Sketch and Learn, we show the image and have glyphs and data associated with it. It would be beneficial for learning purposes to have the player revisit and review the information from the image [35]. Sketch and Learn allows for this, but we had the idea of providing information for each image in stages. For example, say we have an image A with 30 different glyphs and information associated with each. This would be difficult to learn all at once, so we would break up the information into separate levels. For 30 glyphs we could have three separate levels of completeness for the image A. On each level of completeness the player would have to sketch ten glyphs and learn the information for each. We would also not allow the player to complete all 30 glyphs of image $A$ at one time. 
Once the player finishes one level of completeness, he would be forced to complete a certain amount of different images before being allowed to continue to the next level of image A. This mechanic would force the player to revisit images in order to learn all the information about them and help them learn with smaller chunks of data. To test whether the player would learn more using this mechanic would require longer experimental times and more than one session per player.

Another possibility was to implement the Sketch and Learn framework for mobile and tablet computers that use a touchscreen. This would give the player even more of a hands on sketching feeling and the ability to learn everywhere with a mobile device. The problem is that type of development would require some modifications to our interface using the touchscreen. The prospecting mechanics could be used, but the sketching would have to be modified as the player's fingers might block the view of the line to sketch. We would also have to design the Game View for the specific size of the device's screen. Although a mobile application is a good idea, we wanted to test the mechanics in a simpler environment.

We are also aware of some limitations to the current game design. Our prospecting and sketching mechanics rely mainly on images and visual cues within them, so we should consider the limitations of this design. We would not recommend teaching domains for which critical thinking or problem-solving skills are required, since these depend on a deep understanding of the domain that cannot be conveyed by associating small pieces of text to images. For the same reason, this framework does not work for learning complex subjects. For example, trying to teach programming or computer science theory using this method would be difficult, although certain facts might be teachable using images. Therefore the Sketch and Learn framework, at this point in time, is limited to domains involving basic facts and ideas associated with images. This approach is also not reliant on an energy source whereas the Sketch and Learn framework requires a device that uses energy to interact with it. Even though the cost 
of the devices (that are capable of running the framework) is dropping, a significant amount of the population simply cannot afford them. For these reasons, Sketch and Learn would not likely replace the textbooks and traditional teaching methods, but could be used as a companion by students to learn the material.

\subsection{Summary}

We chose to focus the thesis on the design of the gameplay and interaction with the images for a standard desktop experience and to keep the Sketch and Learn relatively simple by not adding an achievement system and different levels of the images. We designed the framework to get the player to focus on the images and learn the details through the prospecting and sketching of the glyphs. The prospecting mechanic encourages the player to explore parts of the image that they might have overlooked and the sketching reinforces the glyph symbols and facts that are associated with each one. The star icons underneath the images represent the number of glyphs that can be found and help the player track progress. The star icons were chosen to provide an element of satisfaction since stars are generally associated with achievement. The stars also allow the player to reconnect the information to the glyph at any time, aiding learning.

We implemented all of the mechanics with the purpose of getting the player to spend more time on each image and learn more details about each one. The following chapter describes the study performed with volunteer players playing the game to test whether the Sketch and Learn framework is effective as designed. 


\section{Chapter 4}

\section{Study Design}

This chapter provides an overview and a detailed description of the study that we performed to test the useability and effectiveness of Sketch and Learn. The study assessed our thesis that, by using a combination of effective game mechanics, we will be able to extend a person's viewing time on images and that they will remember more details because of it. We also wanted to see whether certain game mechanics would help students remember more details. To do this we created a game with images using Sketch and Learn and images laid out with the text beside it as they would appear in a textbook.

The remainder of this chapter details the game design, methods and test session details of the study. The results are presented in the following chapter.

\subsection{The Game}

To obtain the content of the game, we approached an Art History instructor, Cristina Martinez, and asked her to provide images and content that would be relevant to Art History. She provided us with 11 images, listed in Appendix A, and between seven to ten point-form details about each, such as the historical significance of a particular gesture or the artistic significance of a design element. 
With the images and content provided by the Art History professor, we used Sketch and Learn to create some game images. We also created some images using traditional textbook-style techniques that conveyed the same information. The textbook-style pages displayed an image and had all the important information displayed to the left. See figure 12 for a comparison of the two styles.

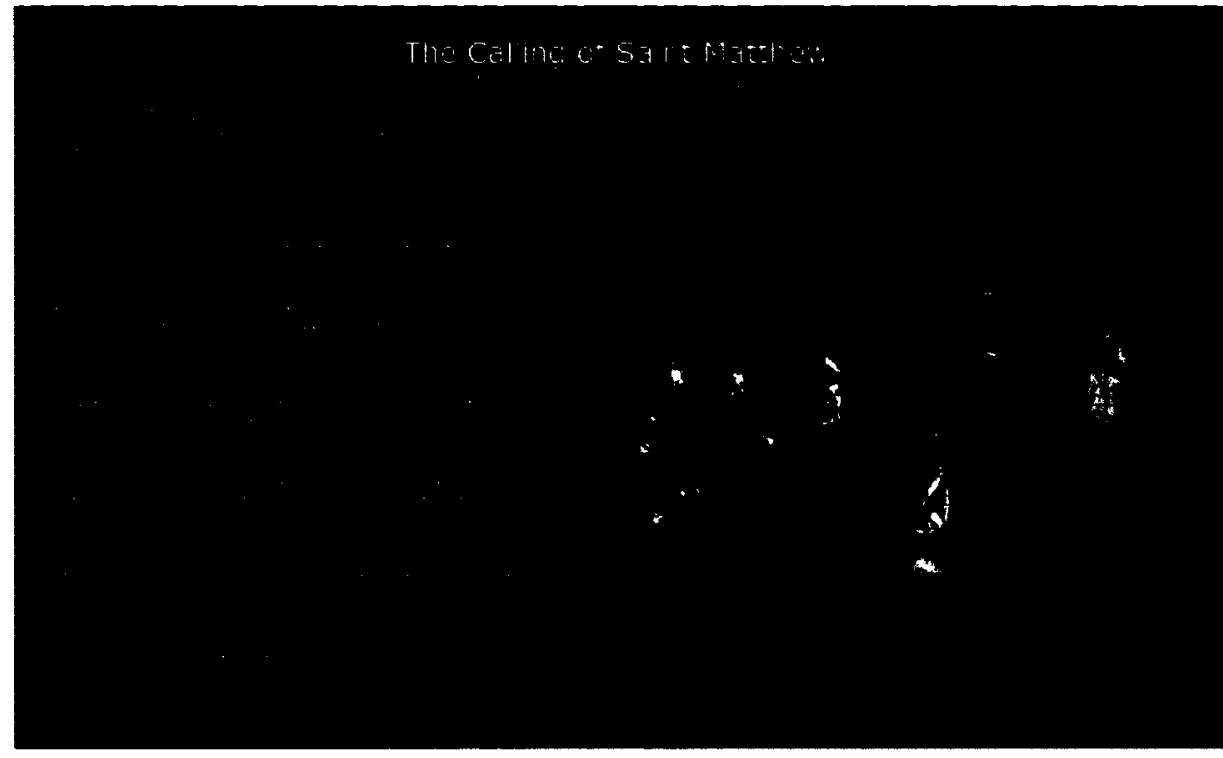

(a) Textbook Style

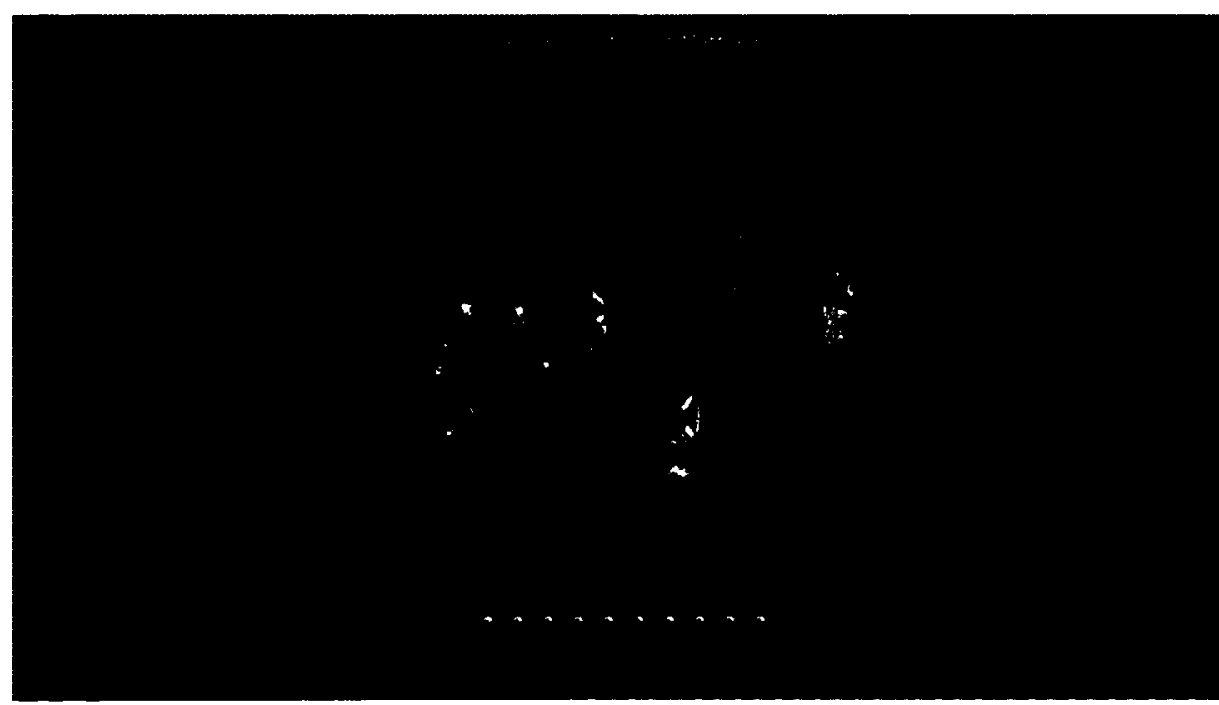

(b) Sketch and Learn Style

Figure 12: Comparison of the styles 
Notice the image takes up approximately a half page on the right and on the left we display all the important information and details associated with that particular image, compared to the larger image of the Sketch and Learn images. These two types of images were alternated in the game as described below.

In the game, we recorded specific data in connection with the images. For the traditional textbook style, the image number and the time spent on the page were recorded. For the game style, the image number, the time spent on the page, and the number of failed tracing attempts were recorded.

We also created an assessment at the end of the game to examine how many details were retained by the participants after playing the game in comparison to the traditional style of learning images. This assessment contained three images, one from the set of images they viewed in a game style, one from the set of images they viewed in a traditional textbook style, and the other was one that they had not seen in the study. These images were presented after the participants had completed a post-game questionnaire, and were asked to describe all the details they recalled or noticed.

\subsection{Hypotheses}

The study was designed to test the following hypotheses:

H1 Participants will spend more time on the Sketch and Learn images than on the textbook-style images.

H1a Participants will spend more time on images that have more glyphs embedded in them than images with a smaller number of glyphs.

H2 Participants will recall more facts and details from the Sketch and Learn images than the textbook-style images. 
If the data supports Hypothesis H1, then Sketch and Learn has met our key goal: to get players to spend more time studying images. However, if players do not remember any facts or details after playing, then our design is not effective. Therefore, the data must support $\mathrm{H} 2$ to show the effectiveness of Sketch and Learn as a learning tool. $\mathrm{H} 1 \mathrm{a}$ is not as important, but supports $\mathrm{H} 1$. If players are spending more time on the images with more glyphs, this would indicate that they are spending more time on the Sketch and Learn images because of the game mechanics of prospecting and sketching, and the pleasure of purification. This idea of purification, where players feels compelled to (or maybe even take pleasure from) completing tasks is discussed in the Theoretical Framework.

\subsection{Methods}

This section will provide the details of the procedures used in our study. We wanted to have an emphasis on the game, so that the users would play and test our game to give us a clear idea of how they enjoyed it, if it promoted viewing the images for a longer, time and if they learned more.

We ensured that each image had a similar number of glyphs, so that we could measure how long participants spent on each image. We also measured how well they performed the sketching task and how well they remembered the details from the two different scenarios. The following sections will describe the participants and how we grouped the participants in the study. Then we will recount how we performed the experiment in chronological order, give the full detail of each step performed, and report how the data was collected. 


\subsubsection{Participants}

We had two main pools of participants. We had 11 participants that were in a firstyear level Art History class and 14 participants that were in university but were not taking any Art History classes, for a total of 25 participants. The age of the participants was between 18 and 25. There were 11 females and 14 males and everyone was able to read and write in English. The participants were recruited by a flyer or word of mouth. Participation was completely voluntary. We received informed consent from each participant, and each participant was given 10 dollars for their participation and time.

\subsubsection{Experimental Groups}

The game involved 10 images. Five of the images were viewed in the game style with the details embedded in the game, for the participant to discover as they interacted with the image, and the other five images were viewed in the traditional textbook style with text on the left and the image on the right. Participants could choose the order that they looked at the images.

The participants were divided into two groups. Group one saw half the images in the game style and the other half in the textbook style. Group two saw the alternate style of the same pictures. See Table 1 for the specific breakdown.

\subsubsection{Test Session Overview}

The study took between 60 to 90 minutes for each participant. They sat in front of a regular desktop computer that had a 17-inch monitor with eye-tracking hardware and software hooked up. They filled out a questionnaire online that included questions about age, gender and art history knowledge. See Appendix B on page 96 for the questionnaire. 
Table 1: Style of images for each group

\begin{tabular}{ccc}
\hline \hline Image & Group 2 & Group 1 \\
\hline 1 & Game & Textbook \\
2 & Textbook & Game \\
3 & Game & Textbook \\
4 & Textbook & Game \\
5 & Game & Textbook \\
6 & Textbook & Game \\
7 & Game & Textbook \\
8 & Textbook & Game \\
9 & Game & Textbook \\
10 & Textbook & Game \\
\hline
\end{tabular}

After we calibrated the eye tracker, they viewed and played the game while the eye tracker was recording data about where they were looking on the screen. When finished, they filled out a post-game questionnaire, online. This included questions about their previous knowledge of the images shown, how they enjoyed the game, and if they enjoyed the sketching.

As the final element of the study, they were shown three images and were asked to describe all the details about them. The following section will go into further detail about each part of the study.

\subsubsection{Test Session Detail}

The previous section provided a chronological overview of the study; in this section, we will provide a more detailed report. At the start of the study, students were asked to fill out a background questionnaire to determine their previous knowledge of video games and art history. We also calibrated the eye tracker to the participant. The calibration required them to look at the screen and follow a blue dot that moved to 
different positions on the monitor. The questionnaire and the calibration took less than five minutes for each user.

Following the calibration, students were verbally given a description of the game and the rules. Due to technical limitations, discussed in the Results section, we informed participants that they should not view the game images more than once. They were also told that they were expected to learn all the information that they would read and that there would be some sort of test at the end.

Then the students were given a practice image to test out the game and when they were ready, they could click on the start game link to start the study. All the participants were able to complete all images within the 45 minutes of allotted time. After this, they were asked to fill out a questionnaire about the game.

Following the questionnaire, participants were shown Figure 40: Masaccio, Figure 41: The Calling of Saint Matthew, and Figure 50: A Bar at the Folies-Bergère (see Appendix). They were asked to describe all the details about each image, everything that they remembered of the two familiar images during the game and anything specific they noticed or concluded about the images. This was all voice-recorded. The session was completed once they finished describing the images. We decided to have the participants fill out the questionnaire before the "test" so they had time to reflect on all the images they had viewed.

\subsubsection{Data Collection}

Data was collected in several ways: through the two questionnaires, through eye movement recorded by the eye tracker, through time spent on the page and, for the game images the number of failed tracing attempts.

The questionnaires were filled out on the desktop computer through an online service called Survey Monkey. The background survey asked about the participants' history with video games and knowledge of art history. This helps us analyze the 
results about their experience with playing games and see if there were any differences between the participants who had previous knowledge and the ones who did not have any knowledge of art history.

The questionnaire following the game asked about the satisfaction with the game, a comparison of the two methods, and enjoyment of certain aspects of the game. The participants were also asked if they had seen any of the images before.

The eye tracker we used was the Tobii 1750 and the software we used to interpret the data was Clearview 2.7.0. The eye tracker works by recording eye movements frame by frame. The software running on the computer interprets where the eye is looking on the screen. This particular eye tracker tracks the movements by using Pupil Centre Corneal Reflection, which involves using a light source to illuminate the eye and a camera to capture the reflections. Under normal test conditions, the accuracy of the placement of the eye is within less than a centimetre [48].

\subsection{Predictions}

We expected that participants would get through all of the images provided in the allotted time. However, we did not expect participants to remember all of the details of all the images. We believed that participants would spend more time on the gamestyle images, but we did not know how much more time. We also predicted that, because they were spending more time viewing the game-style images, they would notice more details and remember more of the information that was given to them through the game.

In the next chapter, the results are presented and discussed. The general feedback during the pilot phase, before the official study, was that the most participants enjoyed learning in this manner, believed they would spend more time playing the game to 
learn compared to a textbook, enjoyed playing the game itself, and found Sketch and Learn easy to use. 


\section{Chapter 5}

\section{Results and Analysis}

In this chapter, we will summarize the main results of the study to see whether the mechanics used are effective in accomplishing our goals. The data pertaining to time spent, details mentioned, facts learned, eye movement and fixation, and the questionnaire-based data will be analyzed in separate sections. We will conclude with an overall discussion of the results and limitations of our study ${ }^{1}$ based on the results of the analysis.

To begin, we had to check the quality of the data. We discovered that two participants would need to be excluded due to comments made during the audio analysis. One person revealed previous in-depth knowledge of a particular image and the other reported a lack of sleep that she said would affect her score. These subjects were excluded from the data analysis, thus leaving us with a total of 23 participants.

Of those 23 participants, 14 of them are male and 9 of them female. The background questionnaire revealed that most of the participants do not play video games on a regular basis. Seven answered that they played zero hours of video games per week, eleven said they played between one and three hours, four played between four

\footnotetext{
${ }^{1}$ One of the limitations we ran into was that we omitted recording the number of glyphs completed by each participant into the database; this was because of how we reset the data after each participant.
} 
and seven hours, and only one participant said they played more than that. Figure 13 shows the breakdown in graphical form.

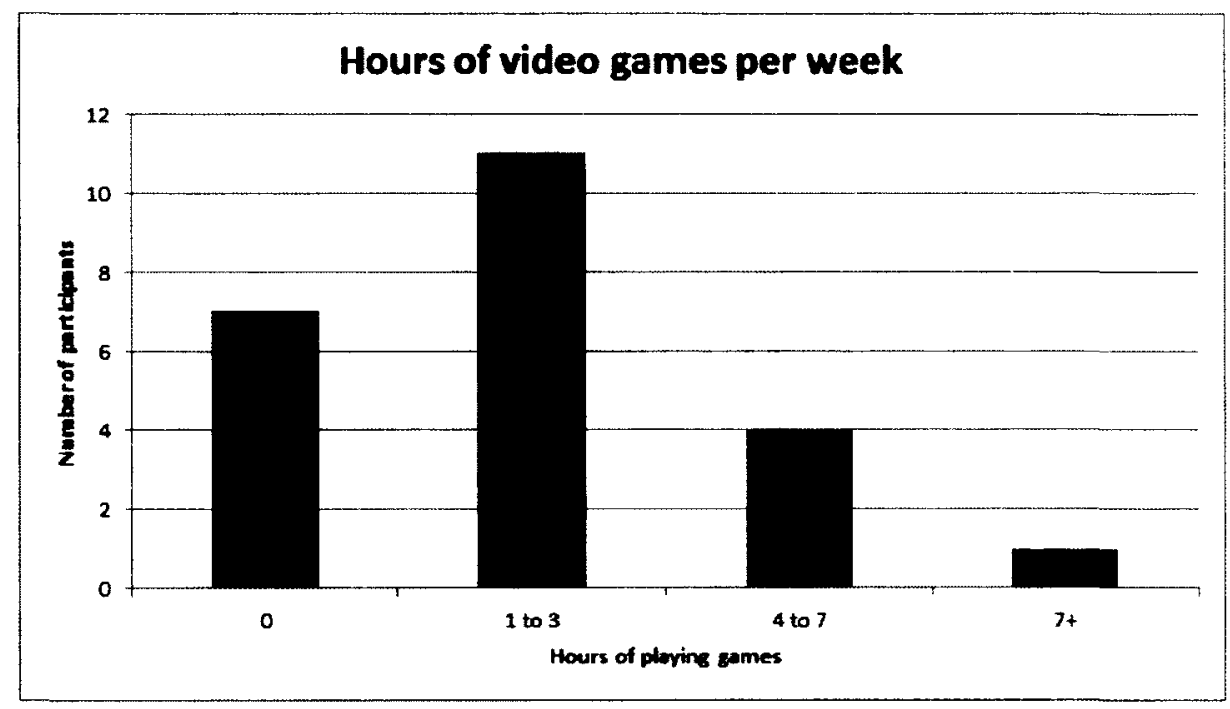

Figure 13: Breakdown of how often participants play video games

We also asked them why they played video games, providing various reasons that they could choose; participants could choose more than one. $64 \%$ said they played for entertainment and $40 \%$ answered to pass the time. Only two participants said they played them to develop their intellect and the rest said that they only played with friend or that they do not play them at all.

The final question was about the participants' previous art history knowledge, where they were given a choice between one for no knowledge, and five for expert. As Figure 14 shows, 11 participants indicated having no knowledge and 12 indicated that they had some knowledge or that they had taken a couple of classes. No participant chose four or five (expert). 


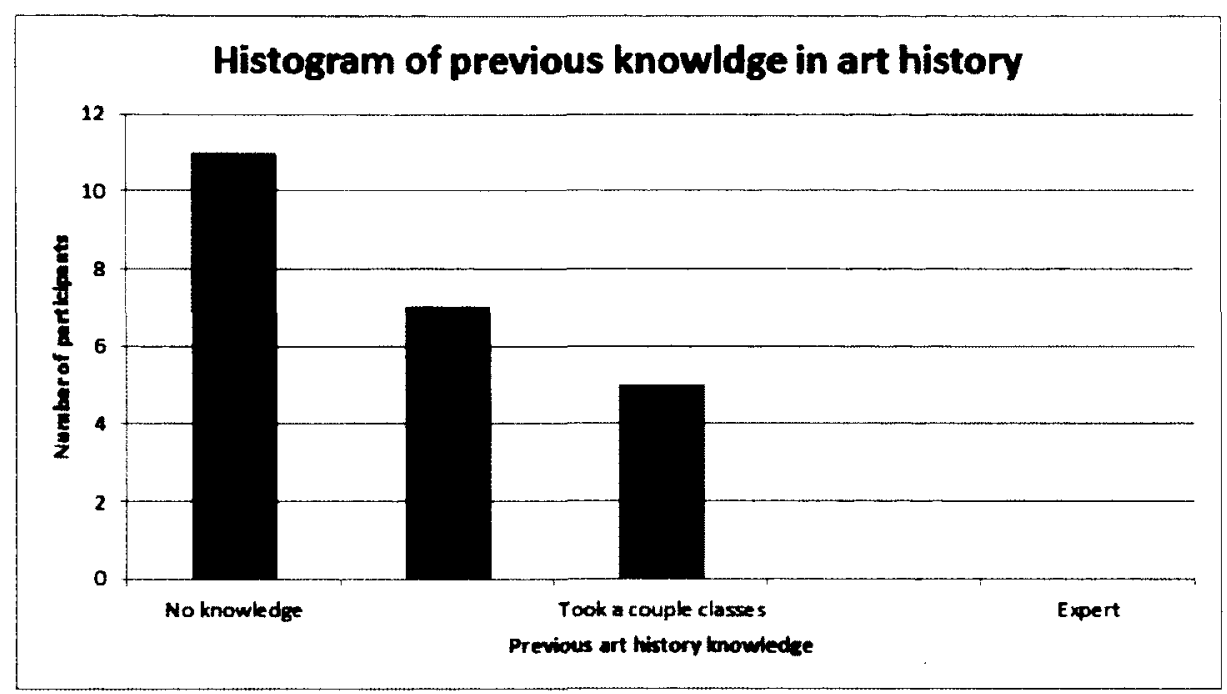

Figure 14: Histogram of previous knowledge

\subsection{Time Spent}

In order to test hypothesis H1, we need to assess whether participants spent more time on the Sketch and Learn images than the textbook-style images in our experiment. We computed the average Sketch and Learn time and textbook time for each participant and compared them. Using a paired t-test in Microsoft Excel 2010, we found the mean, variance, count and P-value. These figures are reported below in Table 2 .

Table 2: Comparison of Time in Minutes between Game Style and Textbook Style

\begin{tabular}{ccccc}
\hline \hline Style & Mean & Variance & Count & P-Value \\
\hline Sketch and Learn & 4.81 & 1.20 & 23 & $<0.0001$ \\
Textbook & 1.42 & 0.39 & 23 & \\
\hline
\end{tabular}

The count in this table refers to our 23 participants. The table shows the mean of the Sketch and Learn images was more than three times the mean of the textbook 
images. This means that the overall average time spent on the image was three times longer, also shown in Figure 15. The variance for both styles is within normal ranges. With regard to hypothesis $\mathrm{H} 1$, our test shows a P-value of less than 0.0001 , meaning that our results did not occur simply due to chance, and that the difference between the Sketch and Learn group and the textbook group is statistically significant. This supports our hypothesis.

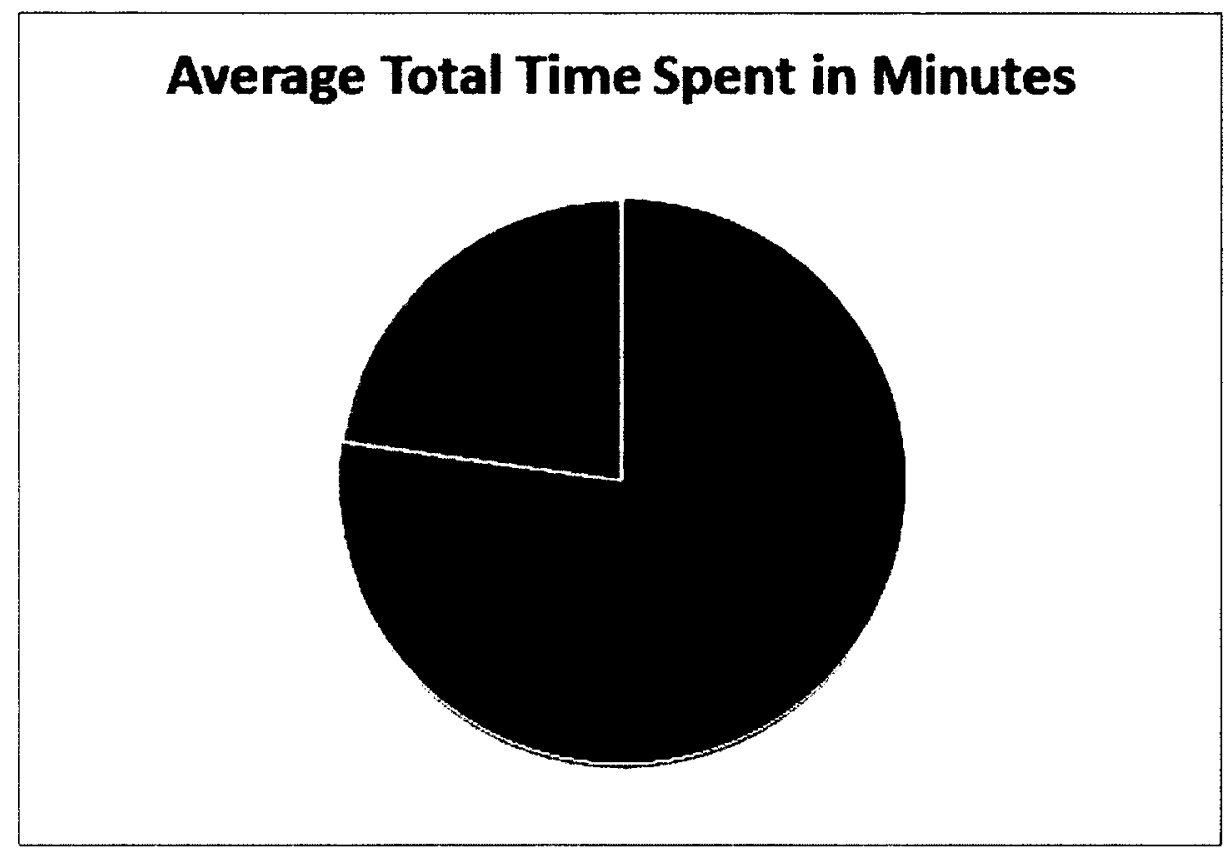

Figure 15: Total average time spent on images for all participants

A comparison of the histograms for Sketch and Learn times and textbook times shows the significant difference between them. Figure 16 shows that most of the participants spent at least an average of three minutes on Sketch and Learn images, while Figure 17 shows that most participants spent less than two. 


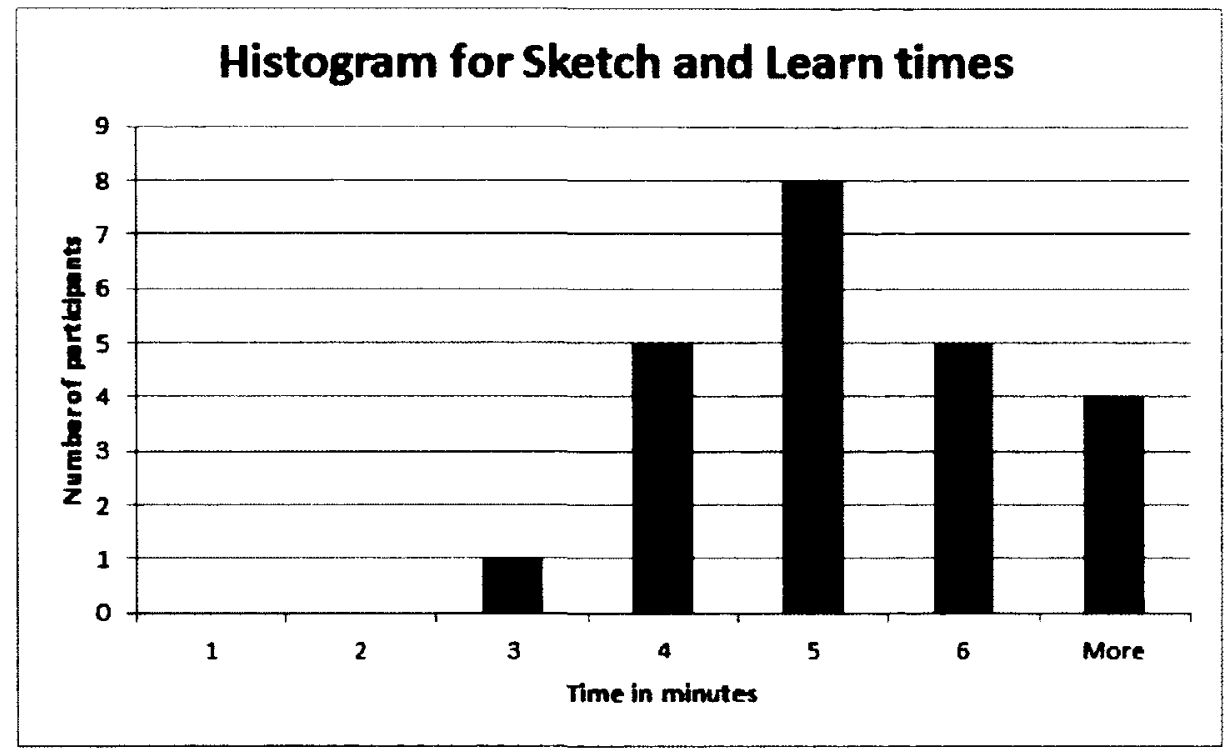

Figure 16: Histogram for Sketch and Learn times

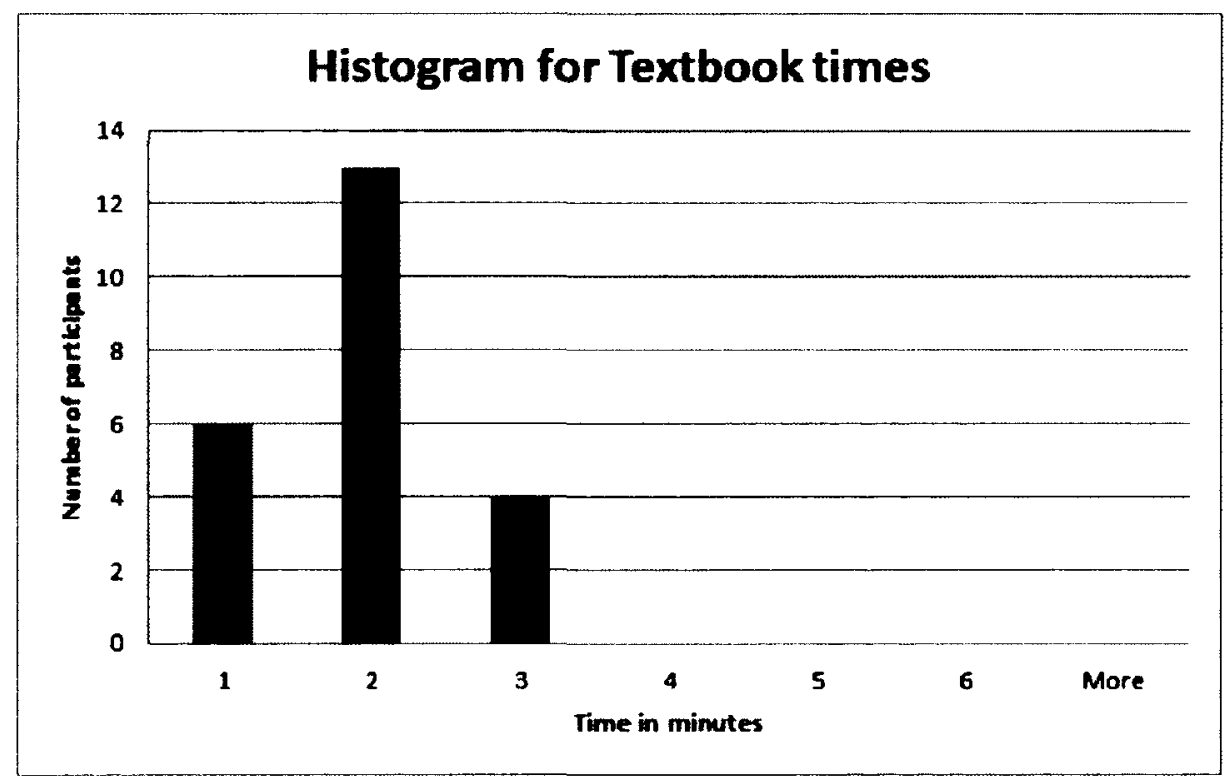

Figure 17: Histogram for Textbook times

We also wanted to see how the art history participants compared to the nonart history participants. Figure 18 shows that participants from art history spent more time on average than non-art history participants in both styles. A paired t-test revealed a statistical significance between art history group and the non-art 
history group for the Sketch and Learn images. The average time spent of the nonart history group was about a minute less than the art history group. There were also no particular comments in the questionnaire that shed light as to why the non-art history participants spent less time than the art history participants; however, we could speculate it is due to a lack of interest in art history and the visual aspects.

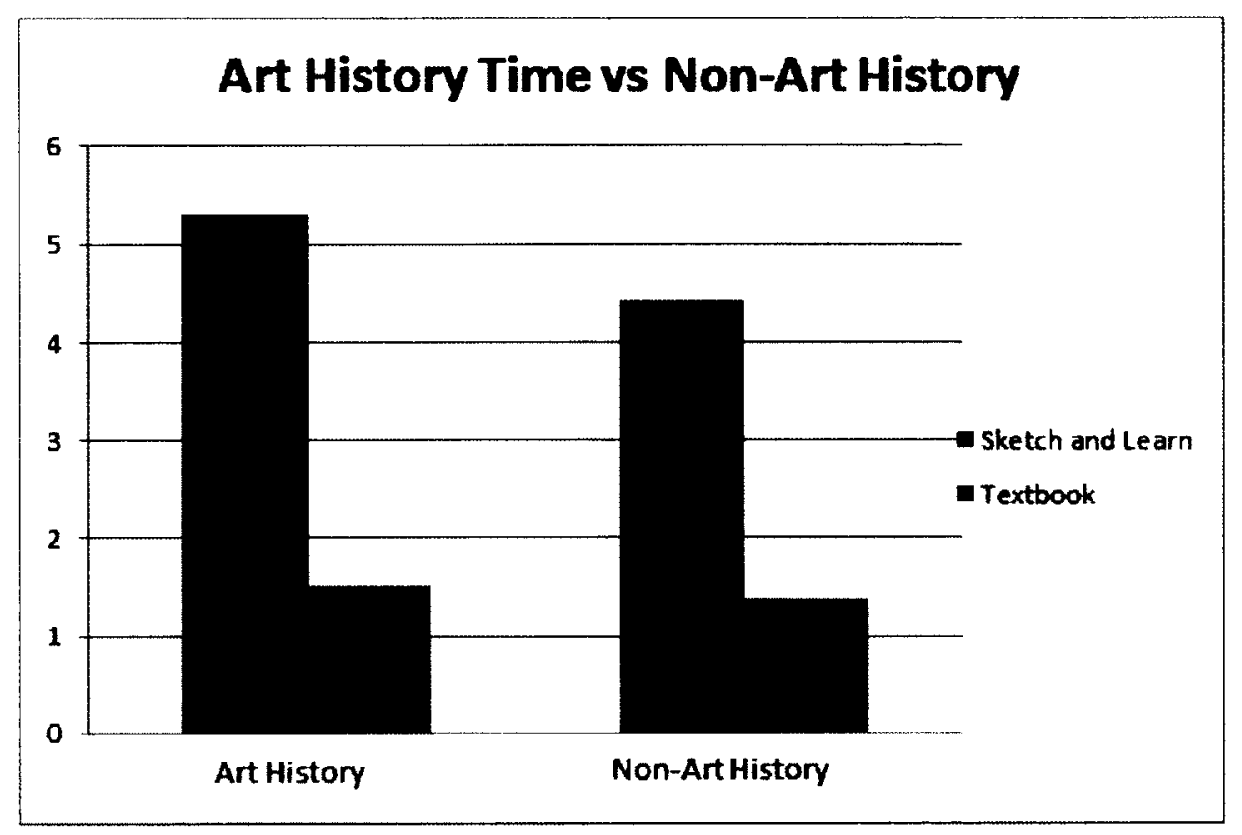

Figure 18: Total average time for art history and non-art history participants

Another paired t-test revealed that no statistical significance between the two groups for the textbook images and that the average times spent were very close with a difference of less than 15 seconds. Therefore, the difference in time spent by the art history participants compared to the non-art history participants is only significant for the Sketch and Learn images. This could be the subject of another study which uses a larger sample size and more detailed questionnaire to examine specifically the different reactions of art history and non-art history students; however, we can speculate that the art history students were more interested in sketching because they are more interested in art. 
Figure 19 shows the average time spent by the number of details/glyphs for both Sketch and Learn and textbook styles. Once again, hypothesis H1 is supported by the fact that the average Sketch and Learn time is greater than the textbook time for the number of details/glyphs. Now we take a look at hypothesis H1a, that participants will spend more time on images that have more glyphs embedded in them than images with a smaller number of details/glyphs.

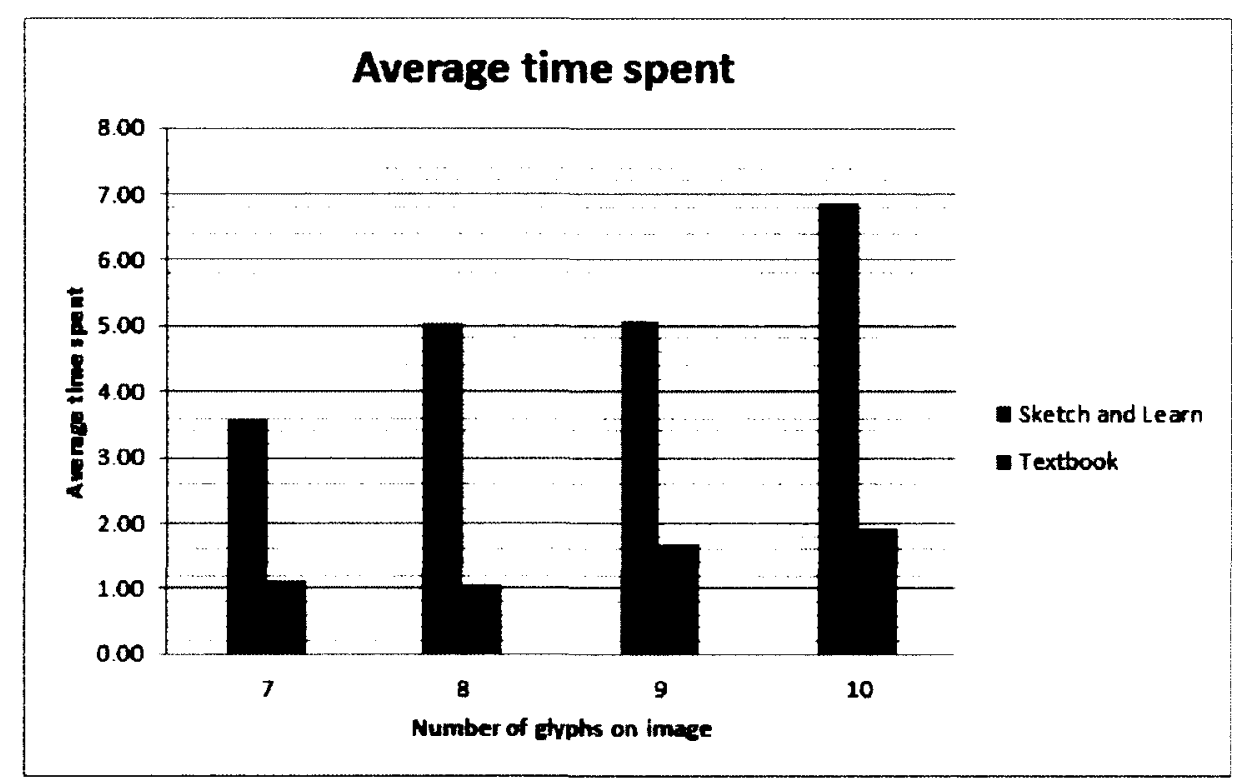

Figure 19: Average time by number of details/glyphs

The figure shows that images with the most details/glyphs (10) have the highest average times spent. Through another paired t-test, we compared the times from the Sketch and Learn images with 10 glyphs to the times of the images with 7 glyphs. For the group of images with 10 glyphs, the average time spent was 6.31 minutes with a variance of 4.84 , and the group with 7 glyphs had an average time spent of 3.30 minutes with a variance of 2.04 . Since the average time spent on the images with 10 glyphs is almost double that of the images with 7 glyphs and with a P-value of less than 0.0001 , meaning that it is statistically significant, the results support hypothesis H1a. 
There are several reasons why having more glyphs on the image would get the participant to spend more time. The most notable reason is that each glyph takes a minimum amount of time to find and to sketch and having more will increase the total time. It is likely that one reason could be that participants feel compelled to finish all the glyphs; this is the pleasure of purification, discussed in the Theoretical Framework. Another reason could be the amount of information to remember. When there are more glyphs and text the participant might take more time to commit all the information to memory.

One reason that makes the comparison difficult is the type and spacing of the glyphs. For example, an image might objects that are spaced throughout which would have the person scanning more and spending more time. On the other hand, when glyphs are closer together, the player can discover several glyphs with one prospect (click). Also, a structural feature such as a focal point might be more difficult to notice than a recognizable object such as a dog.

We come back to looking at hypothesis $\mathrm{H} 1$ and as with any study involving individuals, the average time spent on the images may be distorted by extreme highs or lows. In order to reduce the differences between individuals, we transformed each image time into a standard score. For each participant we took the time they spent on each image $(x)$ and subtracted the mean $(u)$ of all of their images (10 in total). Then we divided that number by the standard deviation (sd) of those 10 images to get their score $(\mathrm{z})$. The formula is $z=(x-u) / s d$. We generated these scores using the scale function in the $\mathrm{R}$ programming language, version 2.14 .2 [47].

Figure 20 shows two histograms of all the participants; one represents all the Sketch and Learn standard scores which the other represents all the textbook standard scores. The figure shows that the standard scores of the Sketch and Learn images are above zero for the most part and the textbook images were mainly below zero. 


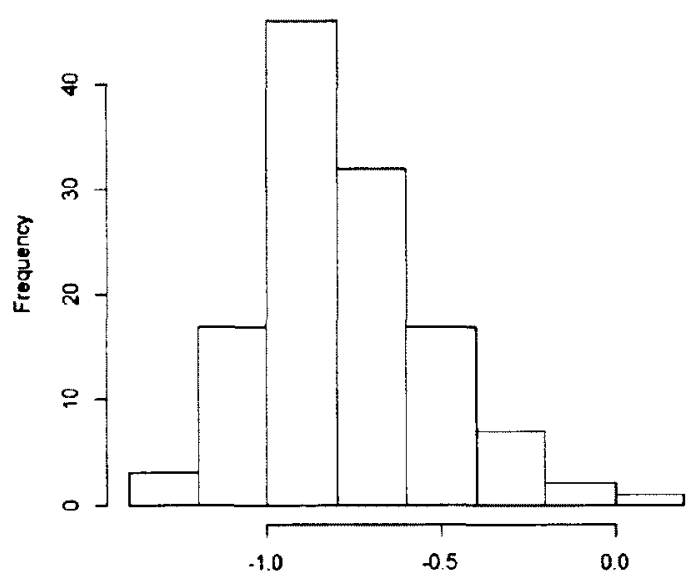

(a) Textbook Style

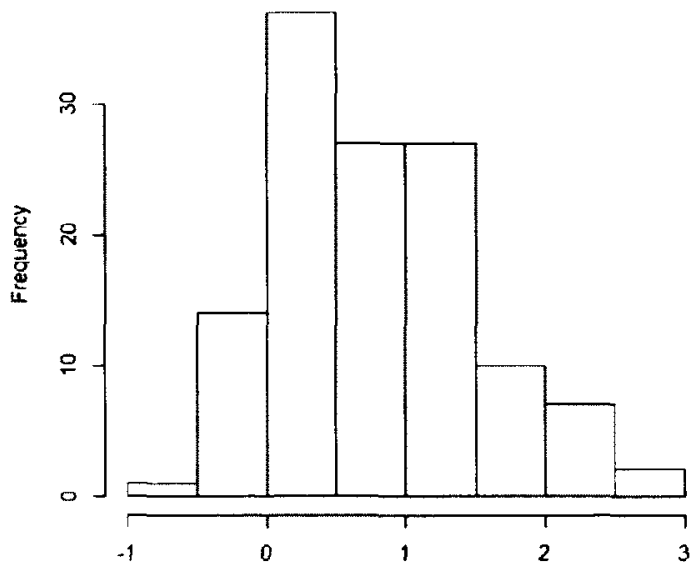

(b) Sketch and Learn Style

Figure 20: Histogram of standard scores separated by style

The histograms show similar distributions of standard scores; the Sketch and Learn scores have a peak on the positive side and show more deviations than the textbook scores which have a peak close to -1.0. We found the average of the Sketch and Learn standard scores to be 0.73 and the average textbook standard scores to be -0.73 , which means that the time spent on the Sketch and Learn images is 1.46 deviations more than the time spent on textbook images. The results of the standard scores support hypothesis $\mathrm{H} 1$ and accounts for the variances between participants.

We also obtained eye-tracking data that can help with the analysis of time spent. The eye tracker keeps a record of each fixation point as an $(\mathrm{x}, \mathrm{y})$ coordinate and the length of the gaze in milliseconds. We were therefore able to create a hot spot plot of one of the images to assess where the participants were looking. As the eye tracker user manual explains, this type of "plot is a powerful way to visualize the gaze behavior of an entire group of recordings" [48].

The hot spot plot below was created by the Tobii Clearview 2.7.0 software and shows where the participants have been looking for the whole time they were on the 
page. The plot includes averaged data from all the participants, based on the amount of times their eyes looked at a specific location on the image. A highlighted area indicated the number of eye fixations the participants viewed that specific location with the colours showing average numbers ranging from no colour, representing little or no fixations, through green and yellow, to red for larger amounts of fixations.

Due to the detailed nature of the eye tracking data and the time required to fully analyze it we chose to analyze only one image, the Calling of Saint Matthew. We chose this image because its hot spot plots are very similar to the aggregate of all the images combined for each of the two styles. It could therefore be considered representative for the purposes of our analysis of eye tracking data. In addition, most of its detail is in the center of the image and less around the edges. This will help us see if Sketch and Learn gets the participants to look at the less interesting details of the images. Another reason is that participants had remembered at least one important fact related to the glyphs based on recorded audio comments made at the end of the game. The image is shown in Figure 21.

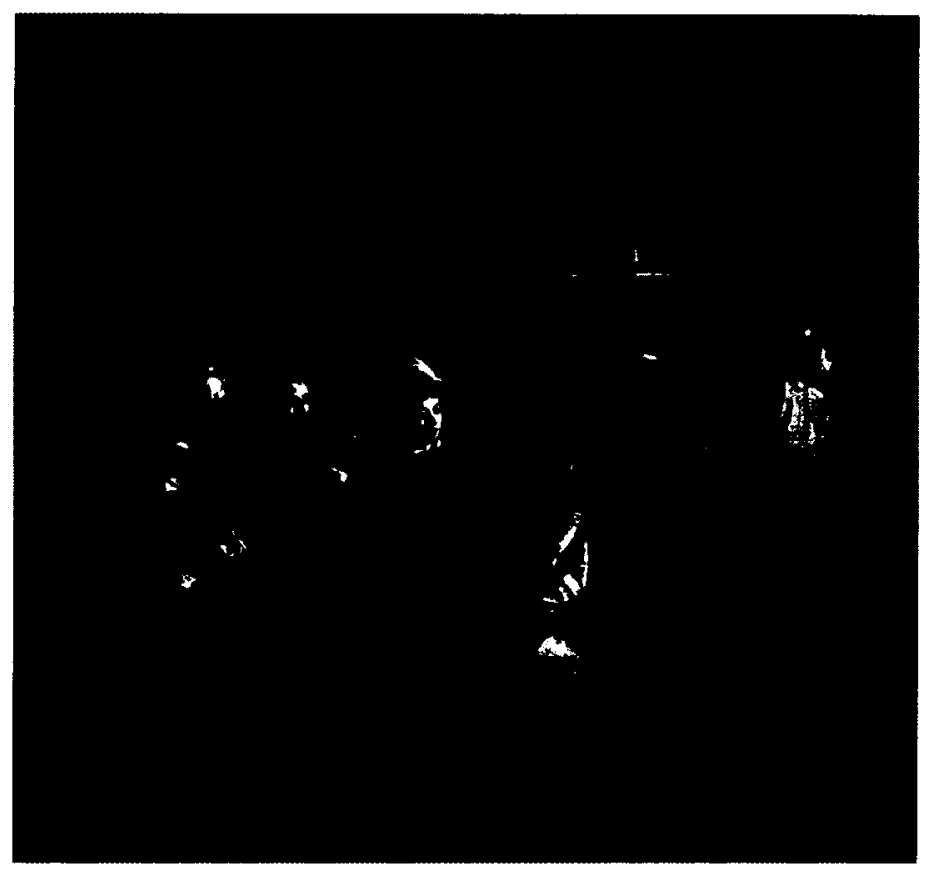

Figure 21: Image of the Calling of Saint Matthew 
The hot spot plot for both the Sketch and Learn style and the textbook style are shown in Figures 22 and 23 respectively. In Figure 22, which is a Sketch and Learn style, the red and yellow spots show participants fixating more on and around the faces in the image. All the green areas, both bright and faint, show that the gaze was also on the feet, the window and other areas of the image, including the corners, possibly showing the participants searching for glyphs. We also see that there were some fixations by the star icons and the text below. This shows participants fixating on the image and reading the text that hovers over the image after the glyphs are traced.

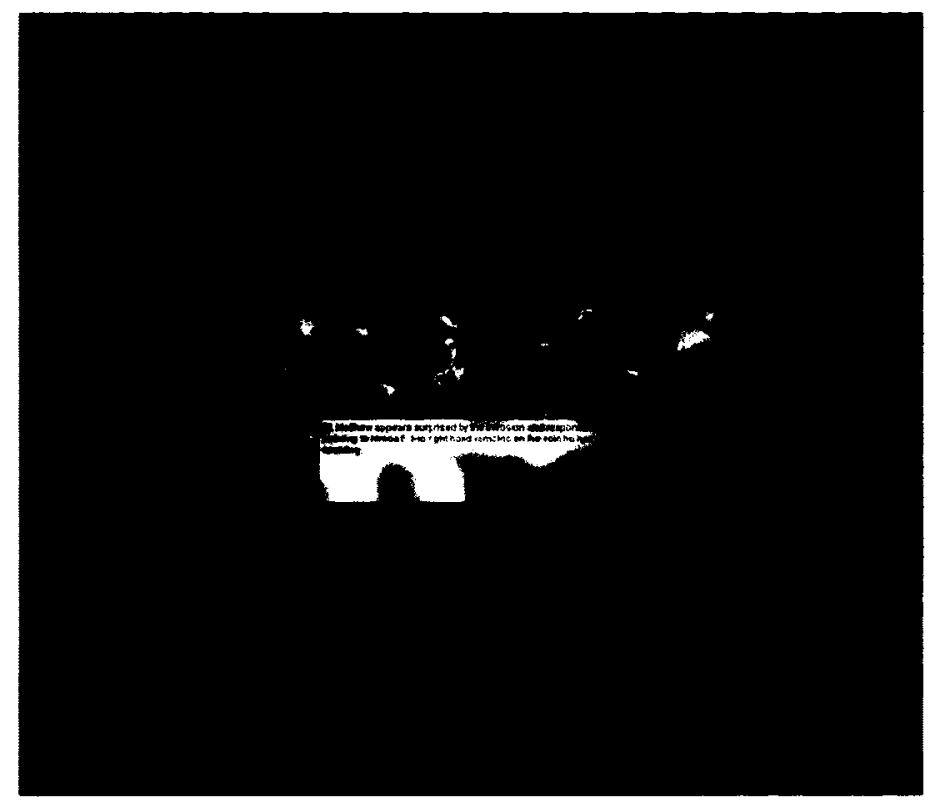

Figure 22: A hot spot image of the Calling of Saint Matthew Sketch and Learn-style page

In contrast, Figure 23 shows participants fixating more on reading the text than observing the image. Although the gaze on the text looks fairly evenly spread out, the gaze on the image is mostly concentrated around the center where the faces are. Major areas of the image, around the window, the feet and the corners, are not studied at all. Notice that there are no red or yellow highlights on the image which indicates 
that participants fixated less than the Sketch and Learn style. This is likely due to the fact that participants spent less time on the style as we showed earlier in this section.

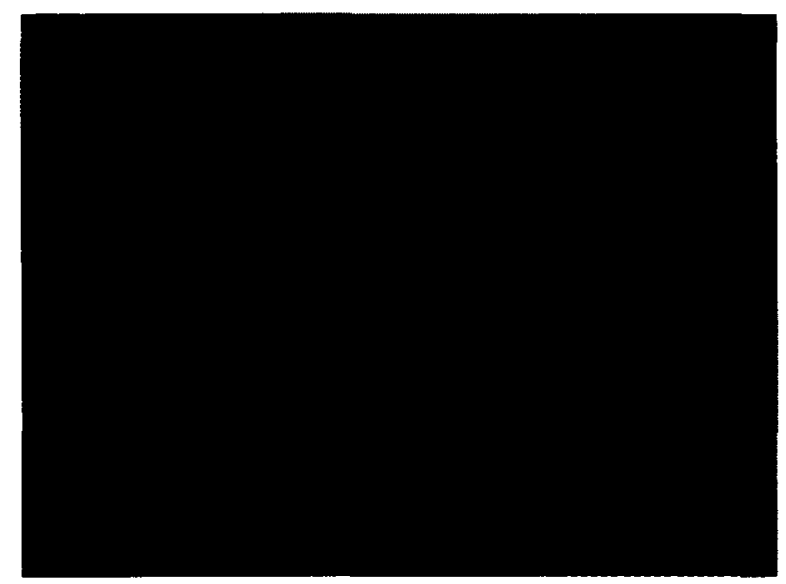

Figure 23: A hot spot image of the Saint Matthew textbook-style page

To assist in the analysis, Figure 24 shows a side-by-side comparison of the two styles. It shows by the red and yellow that participants fixated more on the image in the Sketch and Learn style as expected. It also shows that more areas of the image were viewed.

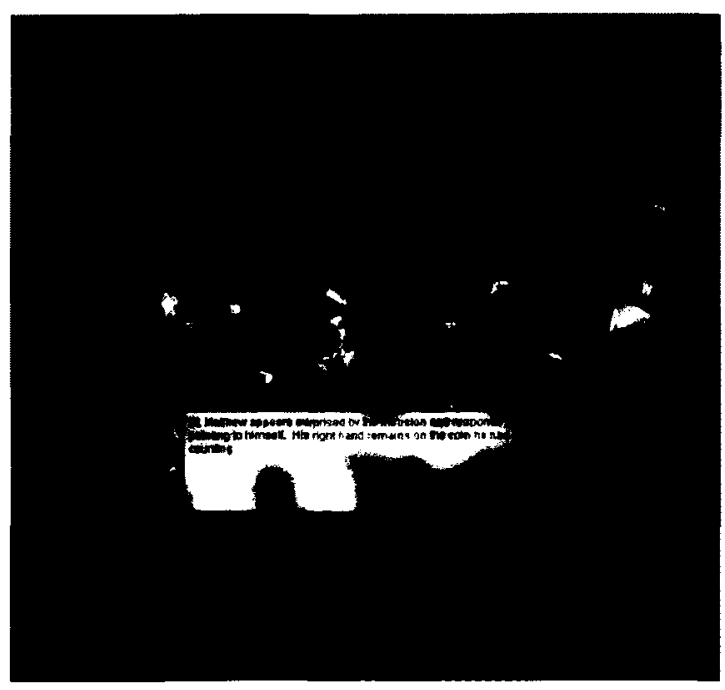

(a) Sketch and Learn Hot Spot

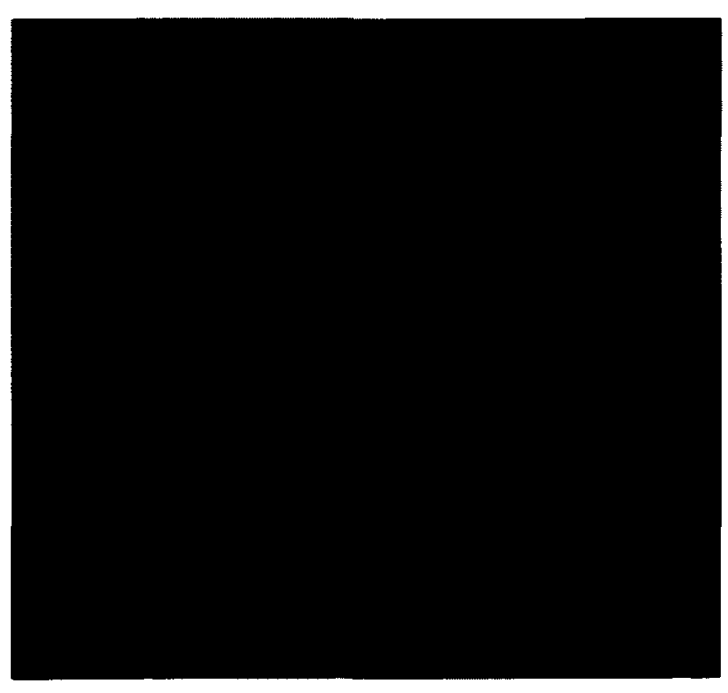

(b) Textbook Hot Spot

Figure 24: A hot spot comparison of The Calling Saint Matthew 
Figure 25 shows the same comparison of the two styles; however, we have normalized the textbook hot spot image to what it would look like if participants had spent the same amount of time as on the Sketch and Learn image by multiplying the fixation count by three, since the data described above showed that they spent three times as much time. The normalized hot spot figure still shows that more areas of the image were viewed in the Sketch and Learn style.

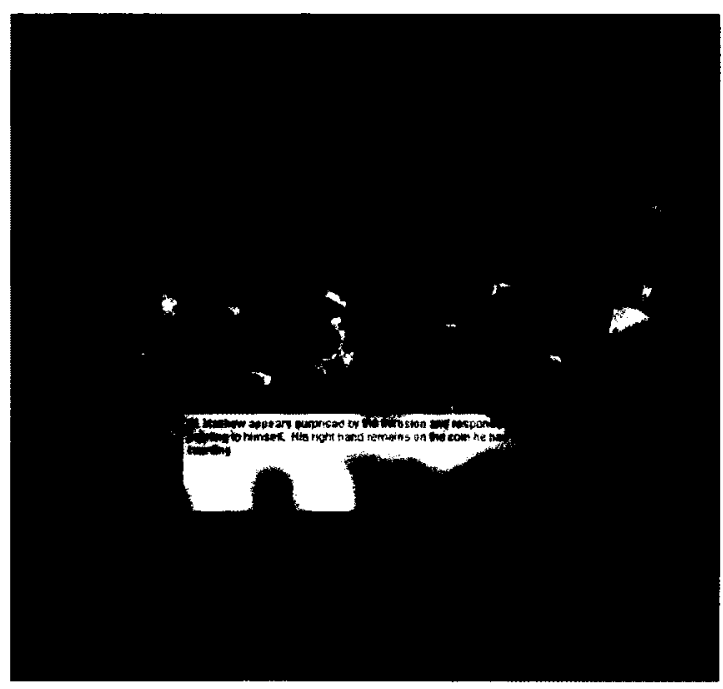

(a) Sketch and Learn Hot Spot

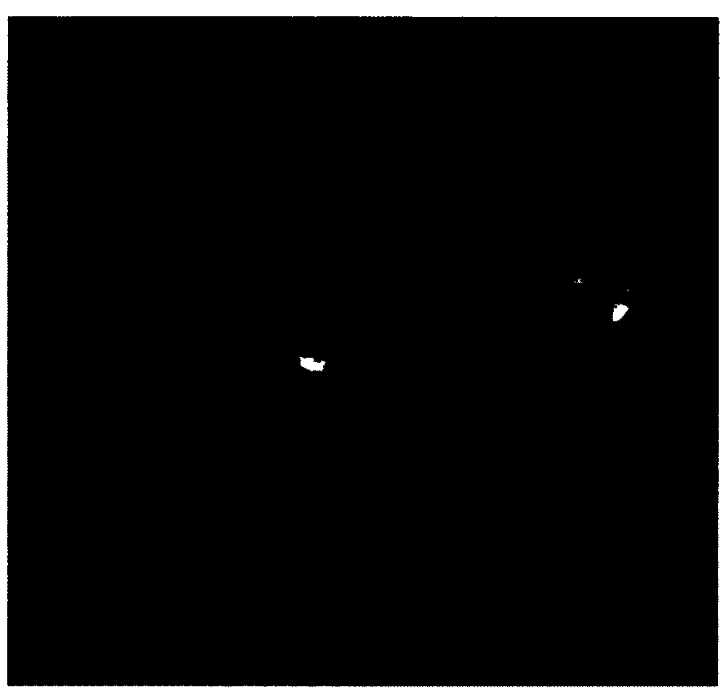

(b) Textbook Hot Spot

Figure 25: A hot spot comparison of The Calling Saint Matthew (normalized for overall time)

Figure 26, in addition to normalizing the time as above, takes into account the time that the text box appears on the image in the Sketch and Learn style. We added $15 \%$ to account for the approximate time for the text box. This percentage was chosen based on a analysis of snapshots to be discussed later. As you can see from these images, the textbook hot spot image still shows less fixations on many areas. An interest in faces is evident in all cases. 


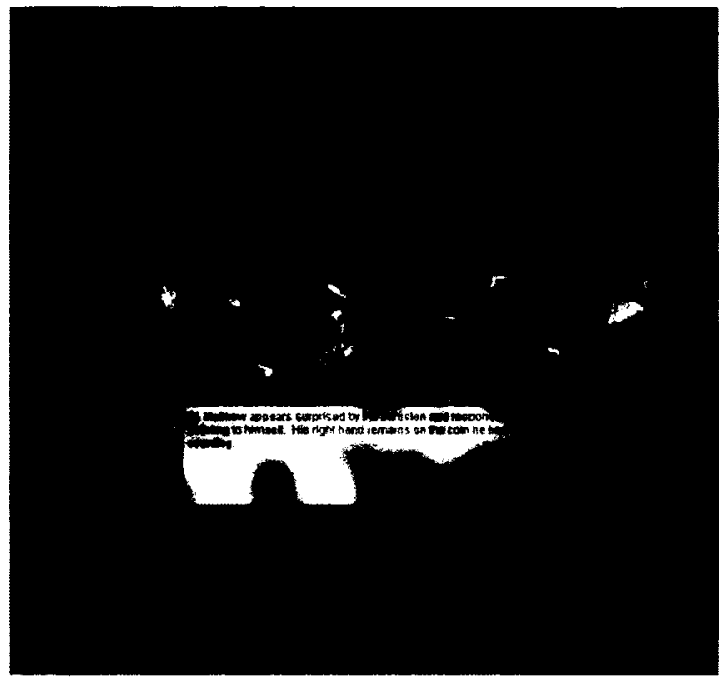

(a) Sketch and Learn Hot Spot

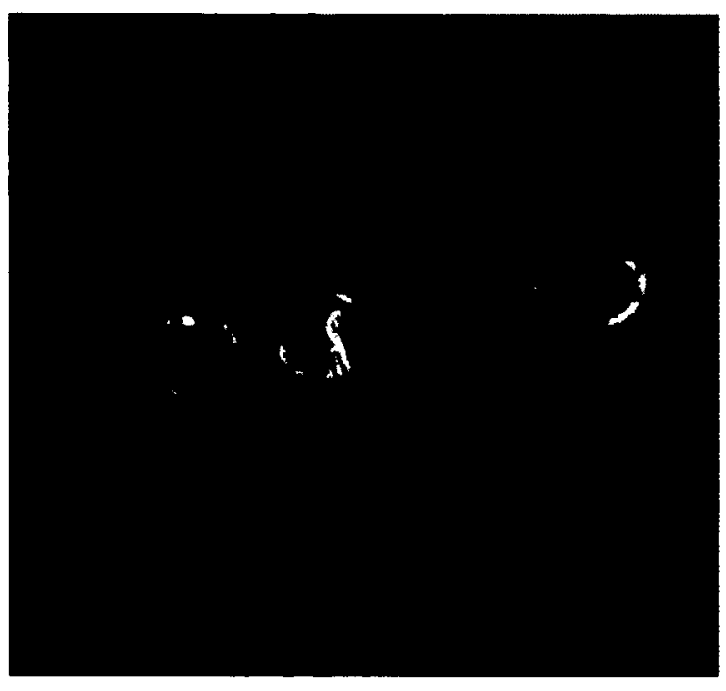

(b) Textbook Hot Spot

Figure 26: A hot spot comparison of The Calling of Saint Matthew (normalized for overall time and textbox time)

Another important piece of information is shown in Figure 27, where we show a side-by-side comparison of the Sketch and Learn style with all the glyph locations and sketching paths. Many fixations occur on and around the sketching paths showing how the sketching mechanic engaged the participants to focus on that area. The green highlights in the corners away from the paths may be due to the participants prospecting in these areas. 


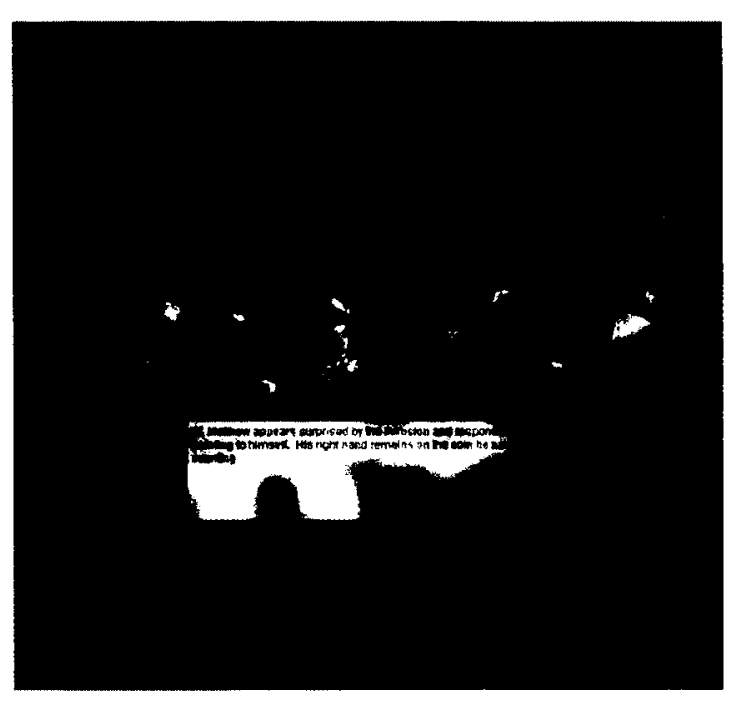

(a) Sketch and Learn Hot Spot

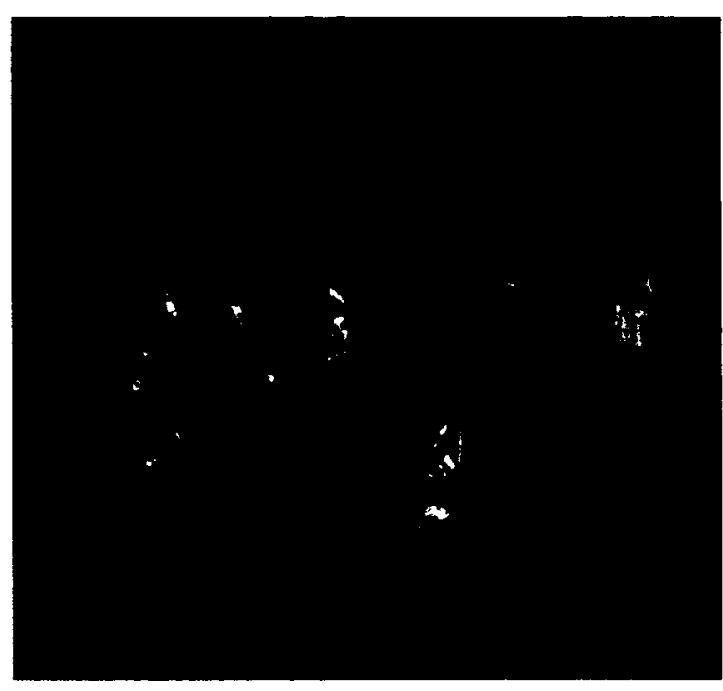

(b) Glyph Locations and Sketching Paths

Figure 27: A hot spot comparison of the Calling Saint Matthew with the glyph locations

In order to seek more insight into how participants played Sketch and Learn images, we analyzed the eye-tracking recordings to see how much time they spent searching, sketching, reading and reviewing. We arbitrarily chose four participants, two non-art history students and two art history students, to give us four snapshots. We also chose a different image for each participant to determine how much the image complexity and glyph locations affected where the time was spent. They all found all the available glyphs in the chosen image. This analysis will help us understand the types of glyphs that cause more difficulty and the effect of the complexity of the image and the glyphs. We also wanted to see if there was any similarity in the percentage of time they spent on the various tasks.

The first recording we analyzed for a non-art history student was 5.22 minutes long and the participant was trying to find the 10 glyphs shown in Figure $27 \mathrm{~b}$ above. These glyphs are of similar size and shape to the glyphs in most of the images and cover more than half of the image, similar to the majority of the images. The average time spent 
on this image was 7.06 minutes so this student spent almost two minutes less. He spent $52 \%$ of the time searching, $29 \%$ sketching, $16 \%$ reading and $3 \%$ reviewing the glyphs and facts (see Figure 28). This student was skilled at sketching and therefore spent less time at that activity. Since this is one of the images used to test the facts recalled, we know that this student remembered six of the glyph details, which is slightly higher than the average for that image (4.56).

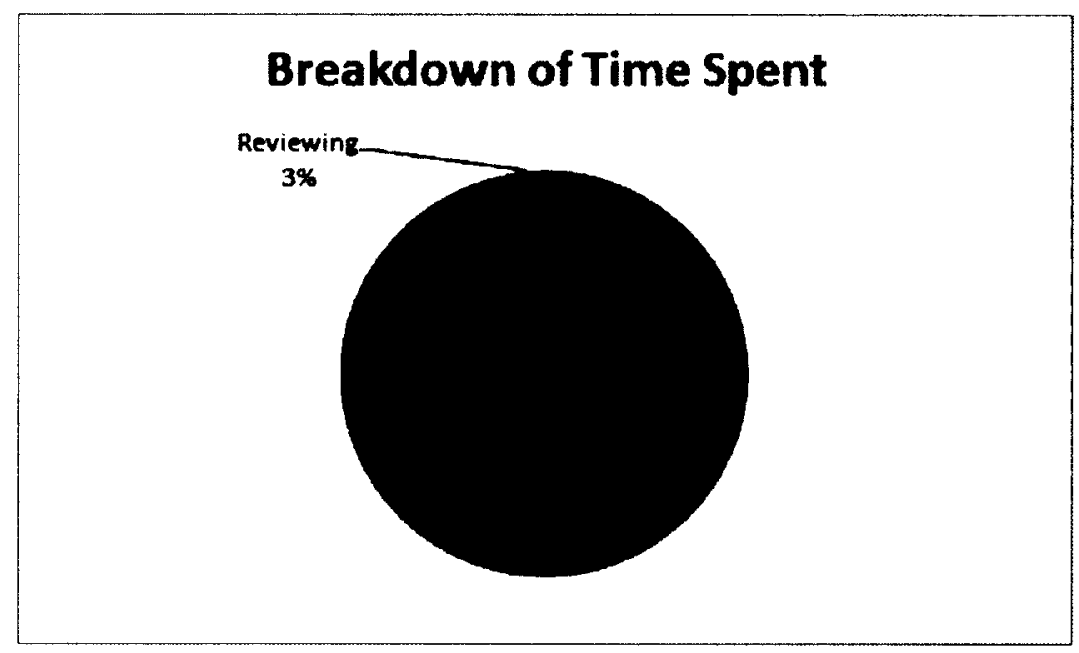

Figure 28: Breakdown of time spent for participant 1

The second recording we analyzed was also for a non-art history student and the image also contained 10 glyphs shown in Figure 29. The participant spent 3.85 minutes on the Sketch and Learn image, a little more than half the average time of 7.36 minutes. The glyphs were spaced throughout the image quite evenly and were associated with most of the distinct objects in the image, making them easier to find. This student spent $38 \%$ of the time searching for the glyphs, $37 \%$ sketching, $16 \%$ reading and $9 \%$ reviewing the glyphs (see Figure 30). 


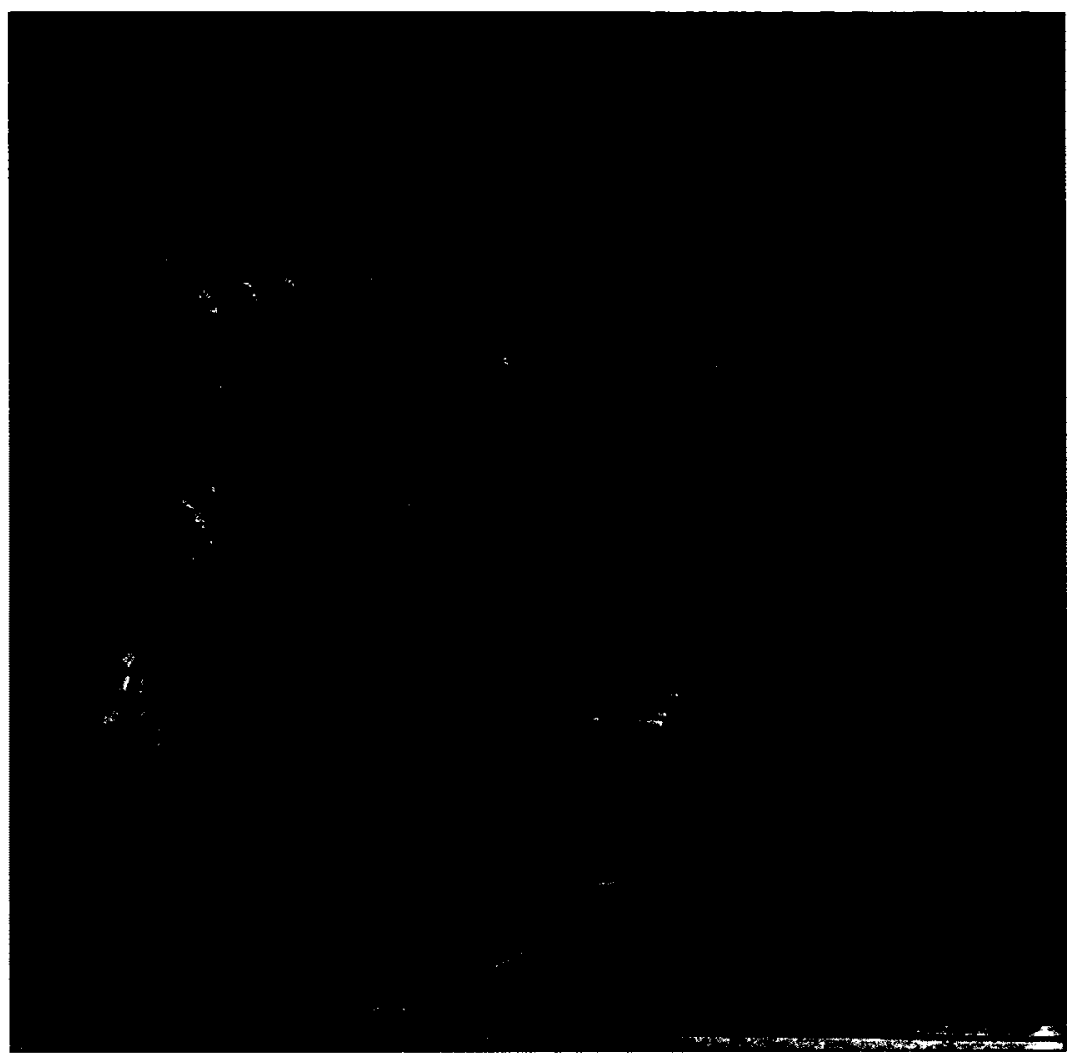

Figure 29: Image viewed by participant 2

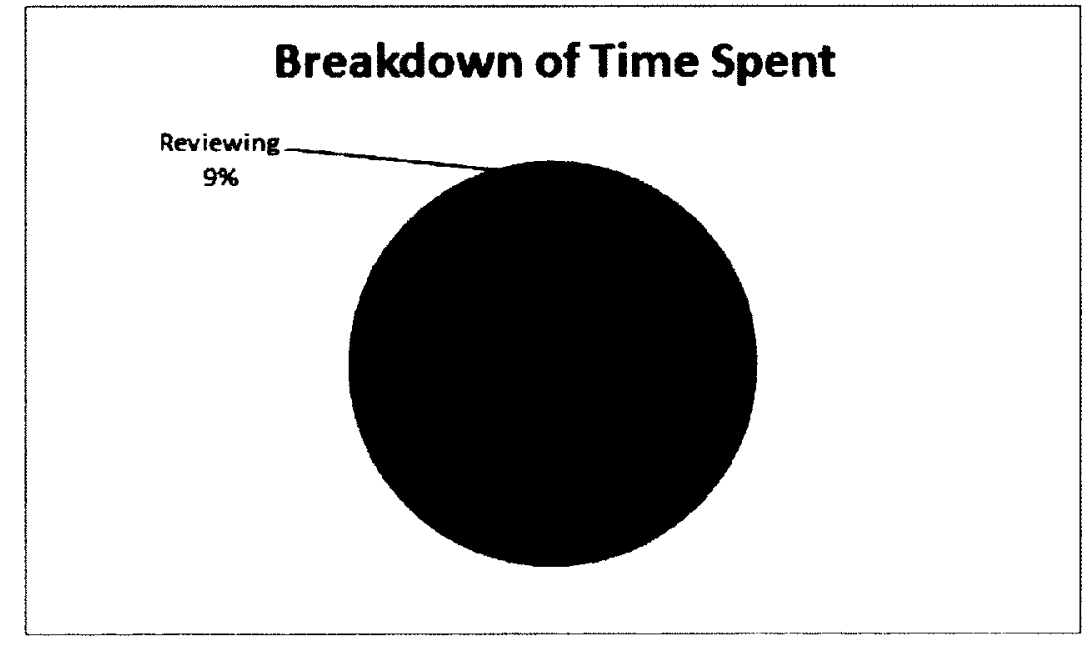

Figure 30: Breakdown of time spent for participant 2

The third recording was for an art history student. The student spent 6.81 minutes on the image, which had many long angular glyphs spread throughout. The average 
time is very close this students time, 6.14 minutes. The glyphs, shown in Figure 31, are concentrated near the center, making it more difficult to distinguish which glyph to sketch. The student spent $43 \%$ of the time searching for the glyphs and $39 \%$ sketching them. The student failed sketching a few times and was distracted by the text; when her eye gaze jumped between the text and the glyph, the cursor strayed too far off the glyph and she had to restart. The last $18 \%$ of the time was spent reading and reviewing (see Figure 32). Of the nine total glyphs, the student recalled four glyph details; this is a bit higher than the average of three.

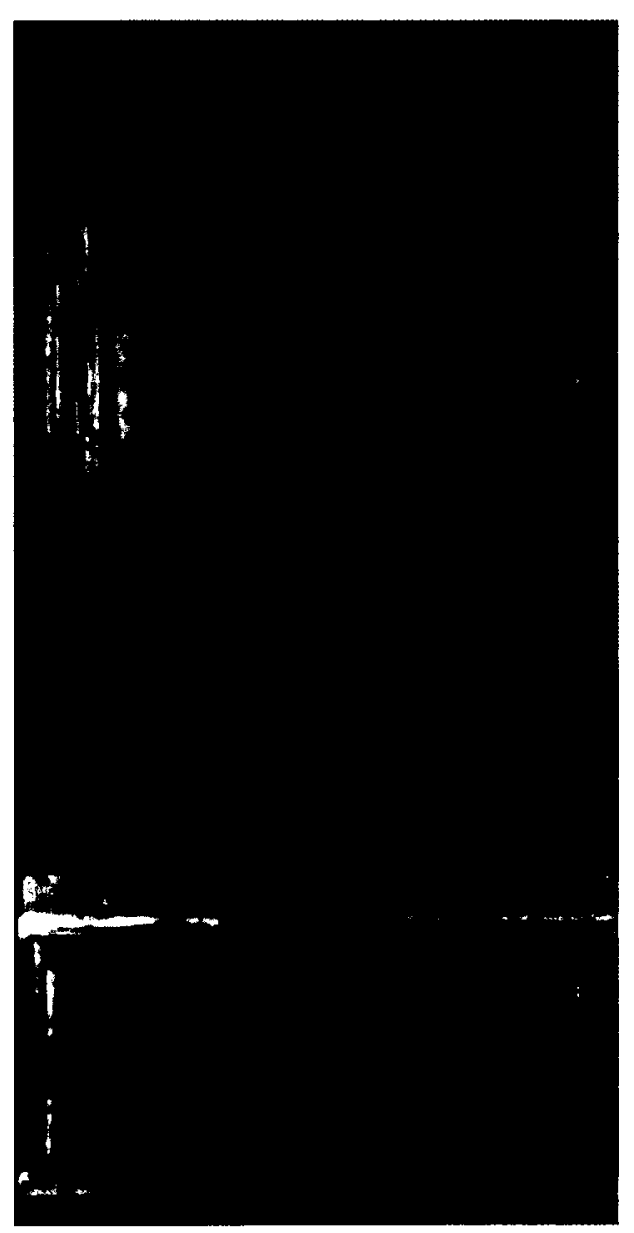

Figure 31: Image viewed by participant 3 


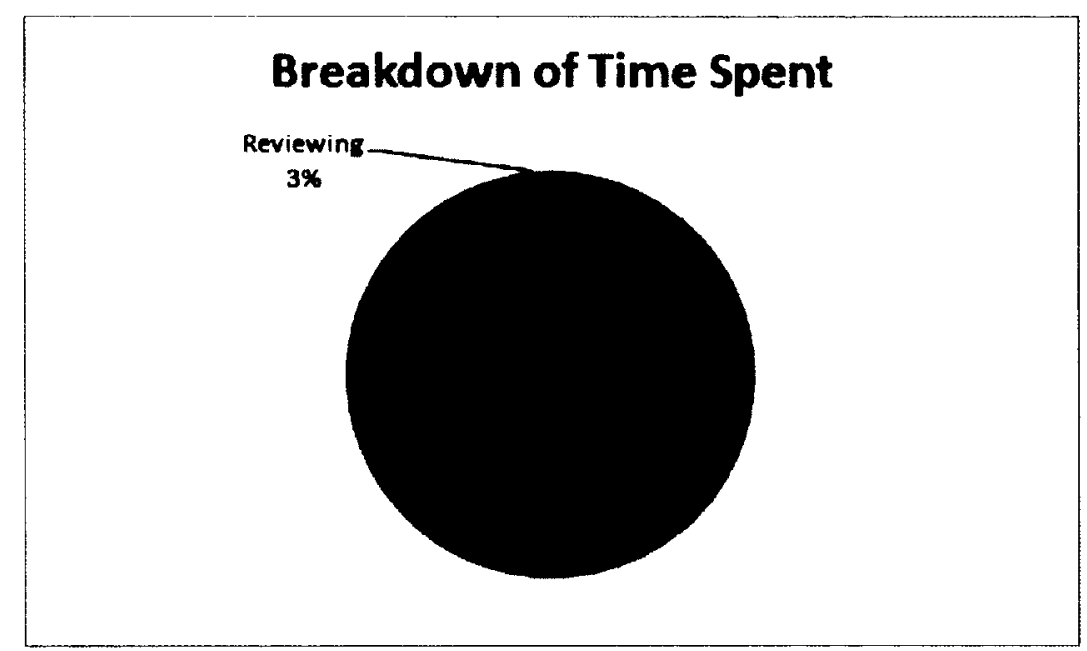

Figure 32: Breakdown of time spent for participant 3

The last recording we analyzed was also an art history student, who spent 4.84 minutes finding the nine glyphs, almost the same as the average, 4.47 (see Figure 33). The breakdown of the time spent differs significantly from the rest of the three recordings described above (see Figure 34 ). Only $11 \%$ of the time was spent searching for the glyphs and $15 \%$ was spent reading the facts. The rest of the time was spent sketching $(64 \%)$; the student did not look at the stars at the bottom. There are a few reasons that could explain this breakdown. The glyphs are all centrally located; with one prospect the student discovered six or seven of them. Also, when the student finished sketching one of them, the start of another glyph popped up. The student would quickly move on to the next glyph and spend less time reading. The two glyphs that were not clustered together are object-based and the student was quick to notice them as glyphs. 


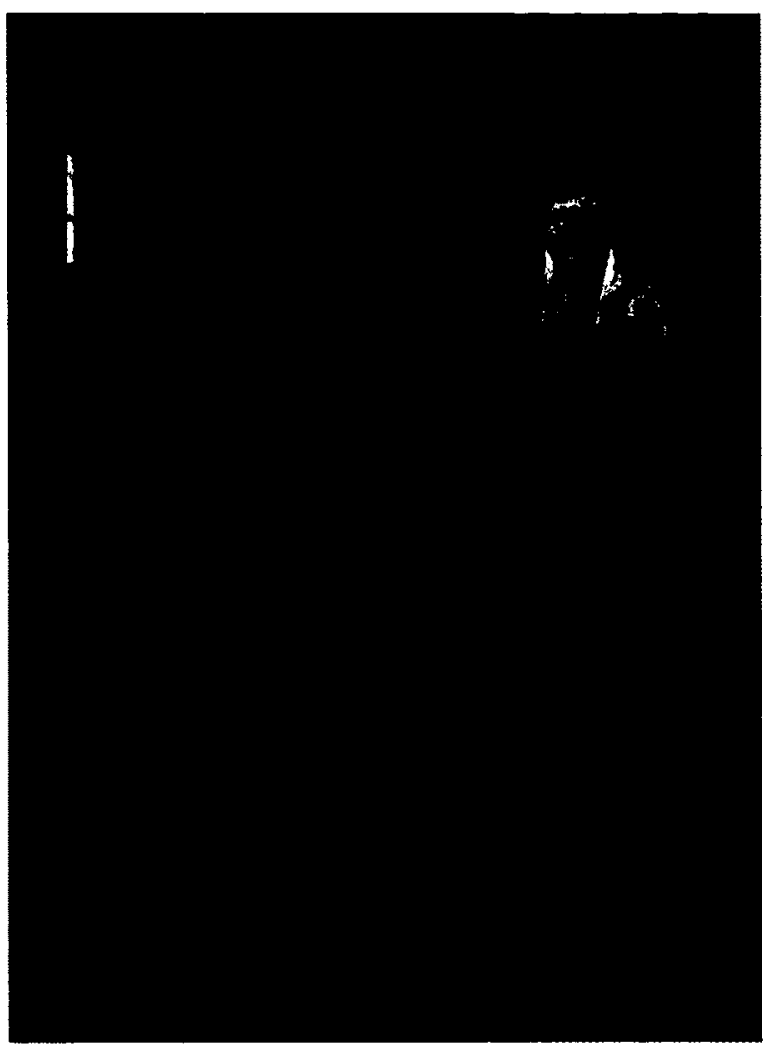

Figure 33: Image viewed by participant 4

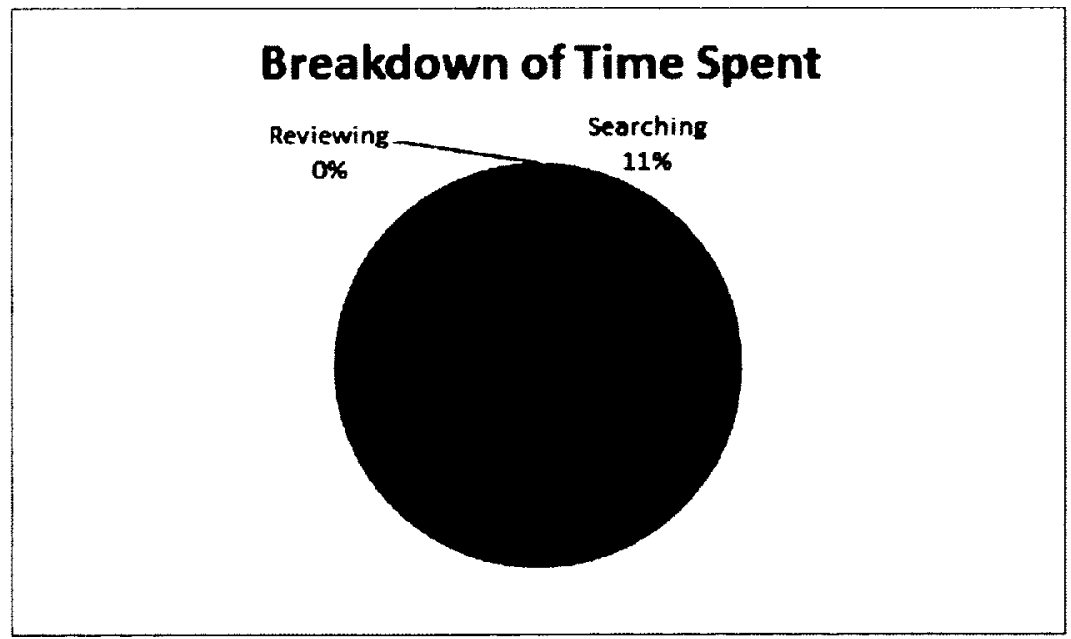

Figure 34: Breakdown of time spent for participant 4

Generally participants spent more than half the time searching and sketching the glyphs with the times for each task roughly equal (except for the fourth recording). 
The rest of the time was primarily spent reading. The time spent searching for glyphs was highly dependant on their size and location within the image and also their proximity to each other. The sketching time was based on the complexity, length and location of the glyph, and also the ability of the player. If the player was distracted easily, then longer glyphs were more challenging. Reading and reviewing depended on how fast the player read and also how familiar the player was with the concepts.

From an observational point of view, participants had more difficulty finding the beginning of the longer glyphs, especially when more than one was in the same proximity. The player looking for the glyphs might find one and think that they had that area covered. However, if a glyph was smaller and appeared directly after the completion of another glyph, then finding the starting point was more obvious and quicker for the player.

Another observation from these recordings was that, although there was no one particular glyph that was more difficult to find, participants often struggled to find the last unknown glyph. This last glyph is inherently tougher to find because the participant has not yet thought of searching in that particular area. Also, since there is only one left to find, then the chances of the participant finding it by accident are a lot less than when there were ten. Although these recordings were just snapshots, they gave us a sense of how the players looked for and sketched the glyphs to help explain some of the other data we have been analyzing.

In this section, we examined the study data relevant to time spent in order to see if it supported our hypothesis H1, that participants will spend more time on the Sketch and Learn images than on the textbook-style images. The data included a comparison of time in minutes between the Sketch and Learn images and the textbook images, and an analysis of the eye tracker data. The data supported hypothesis $\mathrm{H} 1$. 
In addition, a comparison of the amount of time spent on each image allowed us to test hypothesis $\mathrm{Hla}$, that participants will spend more time on images that have more glyphs embedded in them than images with a smaller number of glyphs. This hypothesis was also supported. An interesting fact revealed through our investigation was that art history students spent more time on each Sketch and Learn image than non-art history students. We speculate that art history students enjoyed discovering facts more because they already had some knowledge from other pieces of art; exploring this reason could be the basis of another study. In conclusion our analysis has shown that the game mechanics implemented in Sketch and Learn kept the participants' attention longer than the textbook-style images.

\subsection{Facts Recalled}

The data suggests that the participants spent more time on the game-style images. We now need to see whether they learned more about the details of the Sketch and Learn images than the textbook-style ones. We want to test hypothesis $\mathrm{H} 2$, that participants will recall more facts and details from the Sketch and Learn images than the textbook-style images. As the Study Design section mentions, three images were shown to participants after the final questionnaire. One was from the Sketch and Learn style, one was from the textbook style, and the last was an image they had not seen previously in the study. We used an audio recorder to record the participants' comments on each image which included any details they noticed and any facts they recalled.

To assess hypothesis H2, we listened to the audio recordings from each participant and noted all the times they mentioned general facts about the image and details they remembered from the glyphs. Even if the participants mentioned only part of a fact associated to a glyph, we still gave them a point as it shows that they recalled some 
of the information; however, we did not give them more than one point per glyph fact. Also, we grouped the participant's similar comments into one general point. For each participant, each of the three images has both a general facts recalled score and a glyph facts recalled score, giving us six scores for each participant.

The image that participants did not see in either the Sketch and Learn style or the textbook style gives us a baseline of how many details they might comment on by observation or chance alone. For this image, details associated were also provided by the art history professor and we measured participants' scores in the same way as the other images. Our baseline image had 6 out of the 23 participants recognize two important facts; however, all of these participants were art history students and they indicated that they had seen this image in one of their classes. Also, 9 of the 23 participants noted only one important detail and the details they noted were primarily observational such as a champagne bottle on the table. Finally, 8 of the 23 participants did not mention any of the important information.

In the two images that were seen in the course of the game, we analyzed the responses of participants who had viewed the image in textbook style and Sketch and Learn style. Table 3 shows the results from a paired t-test between the two styles for both images. The count shows that we used the data from all 23 participants. The mean of the scores for the Sketch and Learn style was 4.83 while the mean for the textbook was 2.74 indicating more facts were recalled from Sketch and Learn. The Pvalue was 0.0001 indicating that the difference is statistically significant and supports hypothesis H2. The variance was higher for the Sketch and Learn style because there were extreme scores; two individuals recalled 9 facts, while three individuals recalled two or fewer facts. Also, there is a relation to the time spent which is discussed after the t-tests. 
Table 3: Glyph Facts recalled for both images

\begin{tabular}{ccccc}
\hline \hline Style & Mean & Variance & Count & P-value \\
\hline Sketch and Learn & 4.83 & 4.60 & 23 & 0.0001 \\
Textbook & 2.74 & 1.84 & 23 & \\
\hline
\end{tabular}

To help further analyze the facts recalled, Table 4 shows the results from a t-test between the two styles for image 1, the Masaccio. The mean was 4.00 for Sketch and Learn, which is almost double the mean for the textbook style. Also, since the $\mathrm{P}$-value was 0.006 , the difference is significant and it means that participants recalled more facts from Sketch and Learn, supporting hypothesis $\mathrm{H} 2$.

Table 4: Glyph Facts recalled for Image 1

\begin{tabular}{ccccc}
\hline \hline Style & Mean & Variance & Count & P-value \\
\hline Sketch and Learn & 4.00 & 3.11 & 10 & 0.006 \\
Textbook & 2.23 & 0.69 & 13 & \\
\hline
\end{tabular}

Table 5 shows the analysis of variance between the two styles for image 2 , the Calling of Saint Matthew. The mean for Sketch and Learn was higher compared to the textbook style and the P-value of 0.001 , meaning that the difference between the two styles is statistically significant. This image shows a higher variance than image 1; this can be explained by the high scores of the two individuals we mentioned above. 
Table 5: Glyph Facts recalled for Image 2

\begin{tabular}{ccccc}
\hline \hline Style & Mean & Variance & Count & P-value \\
\hline Sketch and Learn & 5.46 & 5.10 & 13 & 0.001 \\
Textbook & 3.4 & 2.7 & 10 & \\
\hline
\end{tabular}

To find out if participants recalled more glyph facts based on the amount of time, we show Figure 35, a scatter plot with the amount of time spent on each image compared to the number of facts recalled about the details. The red represents the textbook-style; the black the game-style; the circles the non-art history participants; and the triangles the art history participants.

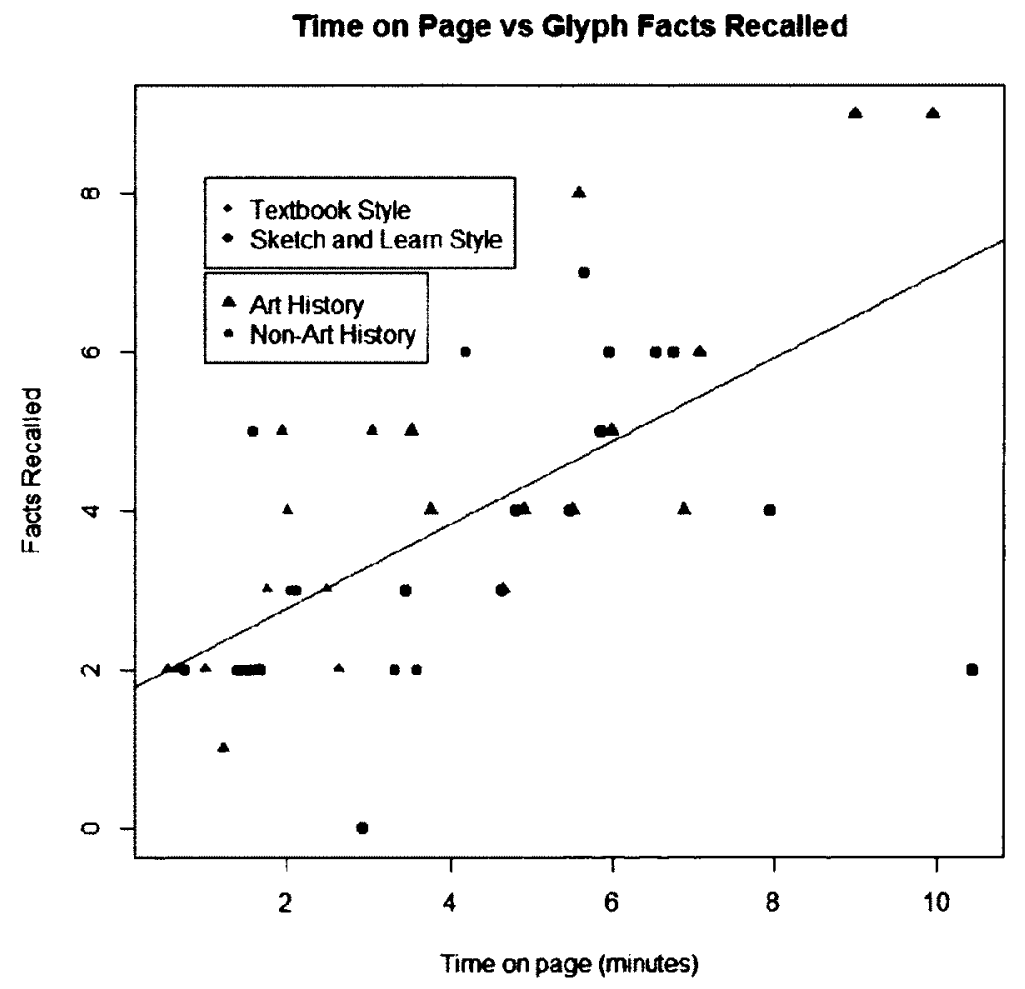

Figure 35: A scatter plot for time and glyph facts 
The least squares line of best fit has a positive slope of 0.53 meaning that for about every two minutes spent on the image, participants recalled one fact. This shows that the more the time spent on both Sketch and Learn and the textbook style, the more facts the participants recalled. There is one main outlier that does not follow the main trend, a non-art history participant that spent a lot of time on Sketch and Learn and only recalled two facts. This participant indicated that he did not understand the material well and had a tough time describing the details. He also appears as an outlier in the next figure for the same reasons.

Other than the one outlier, the Sketch and Learn points, both art history and non-art history, are further up the slope meaning they spent more time and recalled more facts. The textbook style points are lower down the slope and show that the participants from that style recalled fewer facts.

When we remove that one outlier from the equation, the Sketch and Learn data points form a line of best fit (black line below in Figure 36) with a slope of 0.88 which is close to a recall rate of one fact for every minute. The line of best fit for the textbook data points (red line) has a slope of 0.71 , which is less than that of the Sketch and Learn slope. This is a good indication that Sketch and Learn is just as effective as the textbook style and indeed is better since more time is spent learning; however, based on this sample size and the similarity of the slopes, we cannot conclude that Sketch and Learn is a more efficient studying tool. 


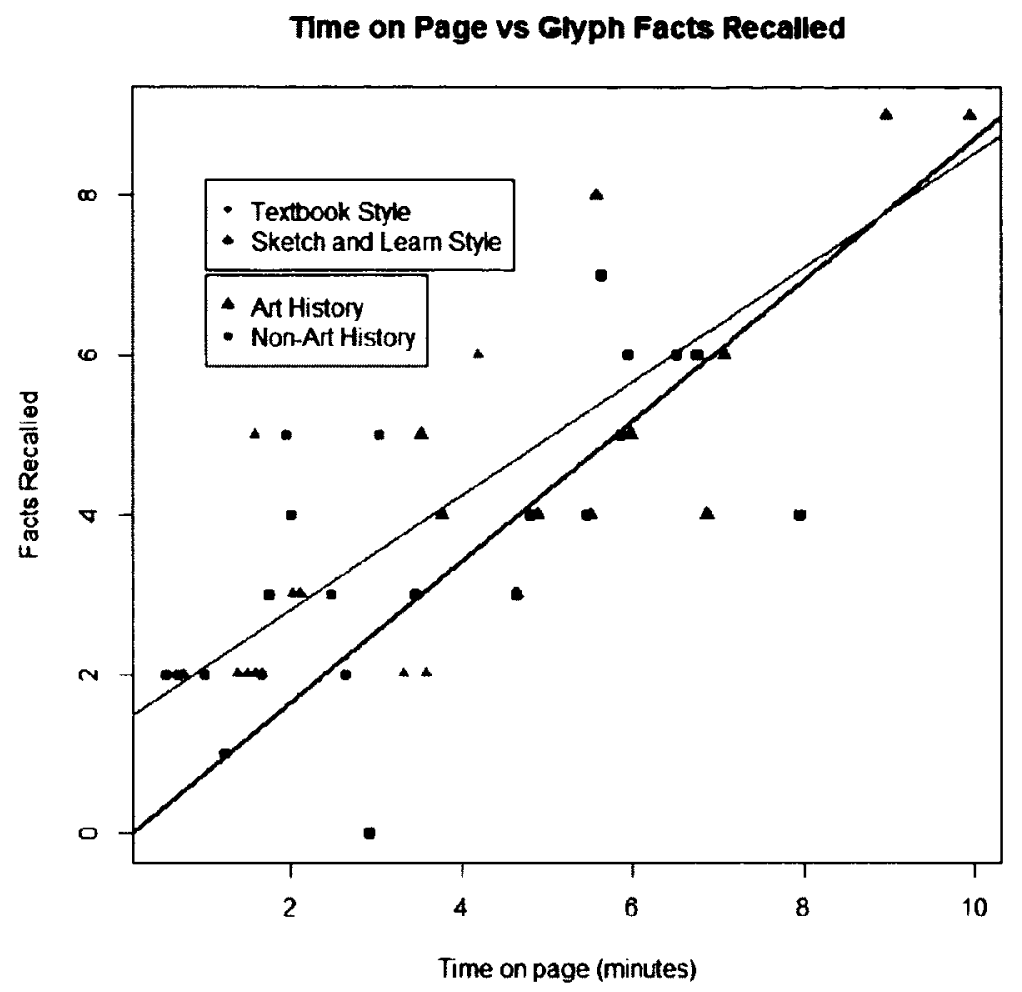

Figure 36: A scatter plot showing lines of best fit without outlier

Another way to analyze this scatter plot is to draw a line between the textbookstyle score and the Sketch and Learn score for each participant. This view is shown in Figure 37 below, where we can visualize the slope for each participants. Notice that we added the outlier back in for this analysis.

The slopes for individual participants show some interesting facts. Three of the participants have negative slopes, meaning they spent more time on the Sketch and Learn image and recalled less facts than on the textbook image. Another participant has a slope of zero, meaning he recalled the same amount of significant facts in both images even though he spent more time with the Sketch and Learn image. These four participants recalled equal or fewer facts in Sketch and Learn style than in the textbook style; however, the other 19 participants had positive slopes and recalled more facts. The minority results may be due to the differences between the two 


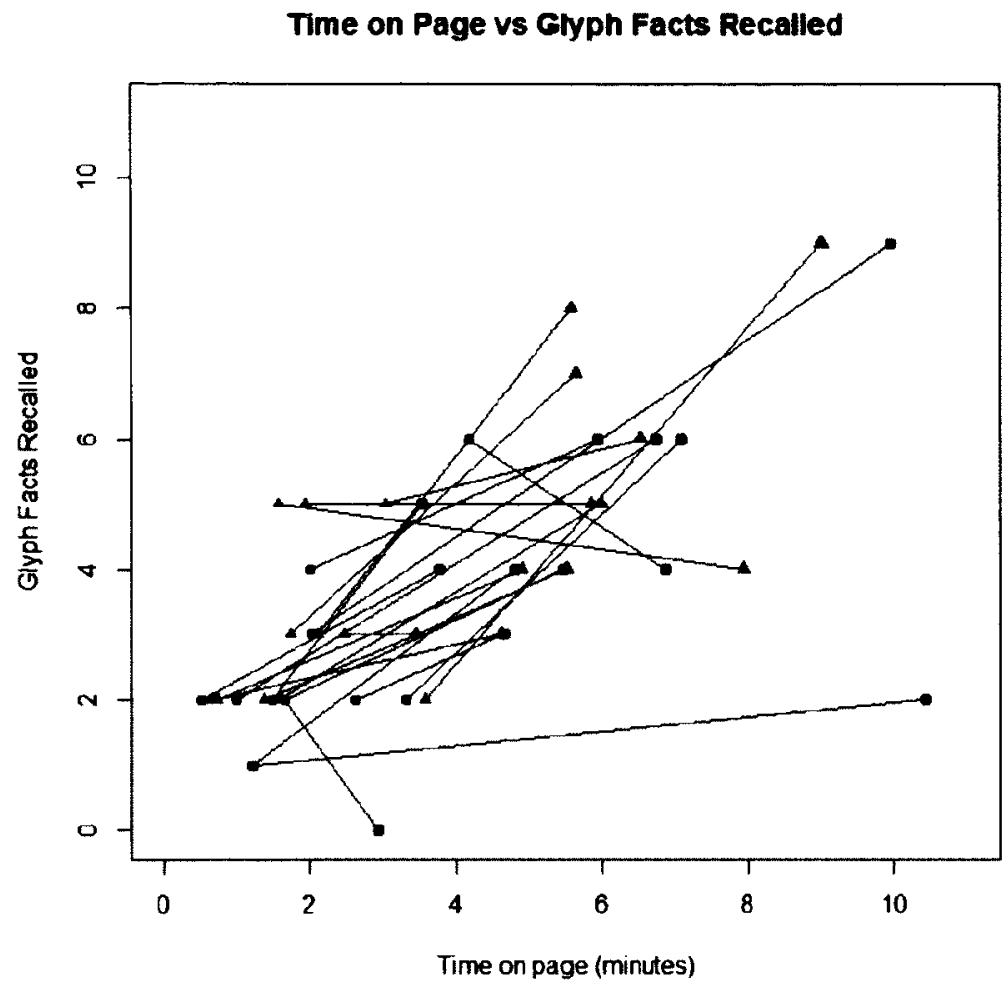

Figure 37: A scatter plot for time spent and glyph facts recalled. One line is drawn for each participant, linking their two scores

images that were tested; one of the images for which a participant had a negative slope featured a crucifix and a grave while the other featured merchants and differences of clothing. We speculate that some individuals might find some of these features more interesting, pay more attention, and therefore, remember more details. We should note that more images and test results might provide more conclusive results.

Since participants also noted details that were not part of the content provided to them, we can also calculate the time spent compared with all the details mentioned, both glyph facts and general details combined. Figure 38 shows the plot and the line of best fit, which has a slope of 0.52 . The data and slope indicate a similar result: that is, the more time the participant spent viewing the image, the more details he will mention, showing that he has gained a higher degree of familiarity with the 
image and a heightened awareness of its features. This is important to note since it demonstrates that our objective of increased learning has been achieved.

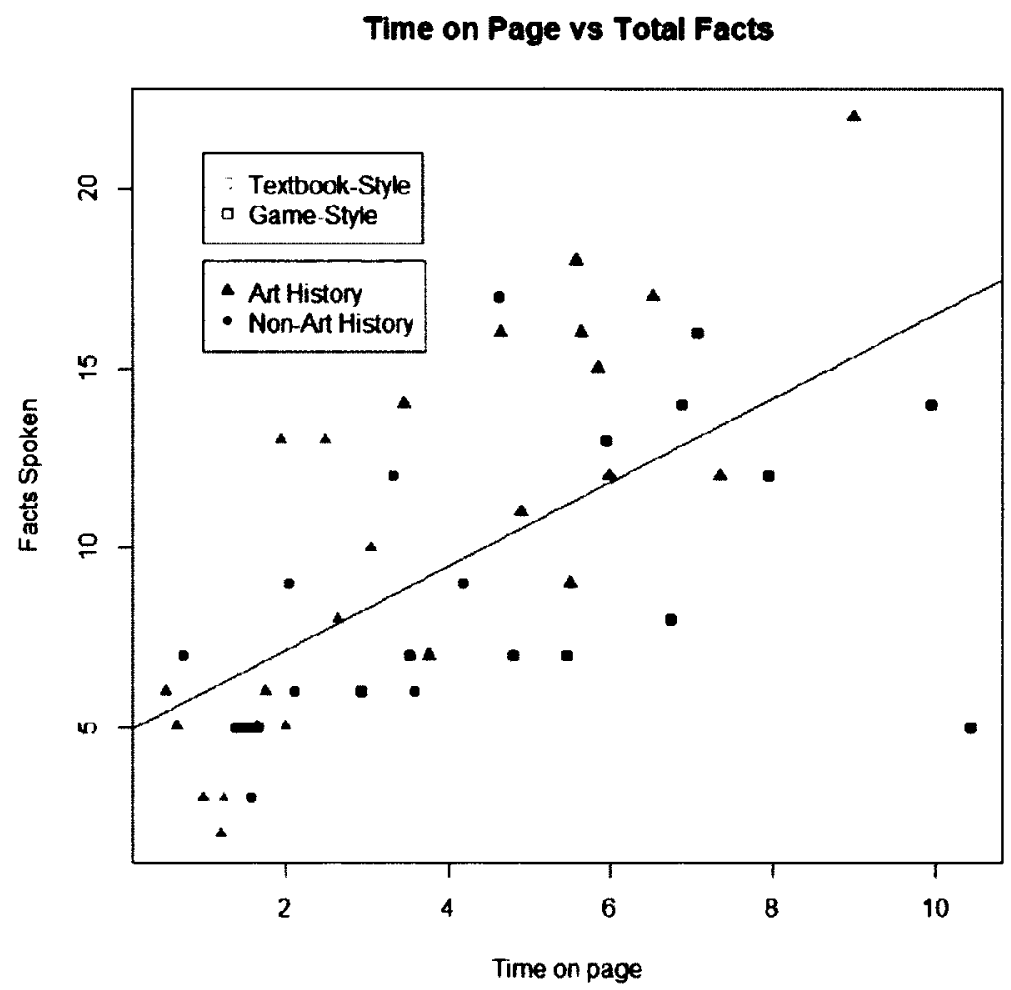

Figure 38: A scatter plot for time and all facts spoken

Since we have the same data for the baseline image, we wanted to see if certain participants were more talkative than others. To do this, we checked the relationship between each of the three images for the number of facts described by each participant. We found that there was very little correlation between any of images, although we still speculate that some individuals are more talkative than others.

For the two images each participant had seen before the final test, we repeated the same analysis as above, drawing one line between the two scores, shown in Figure 39 below. The figure shows that each participant has a positive slope, describing more details in Sketch and Learn. 


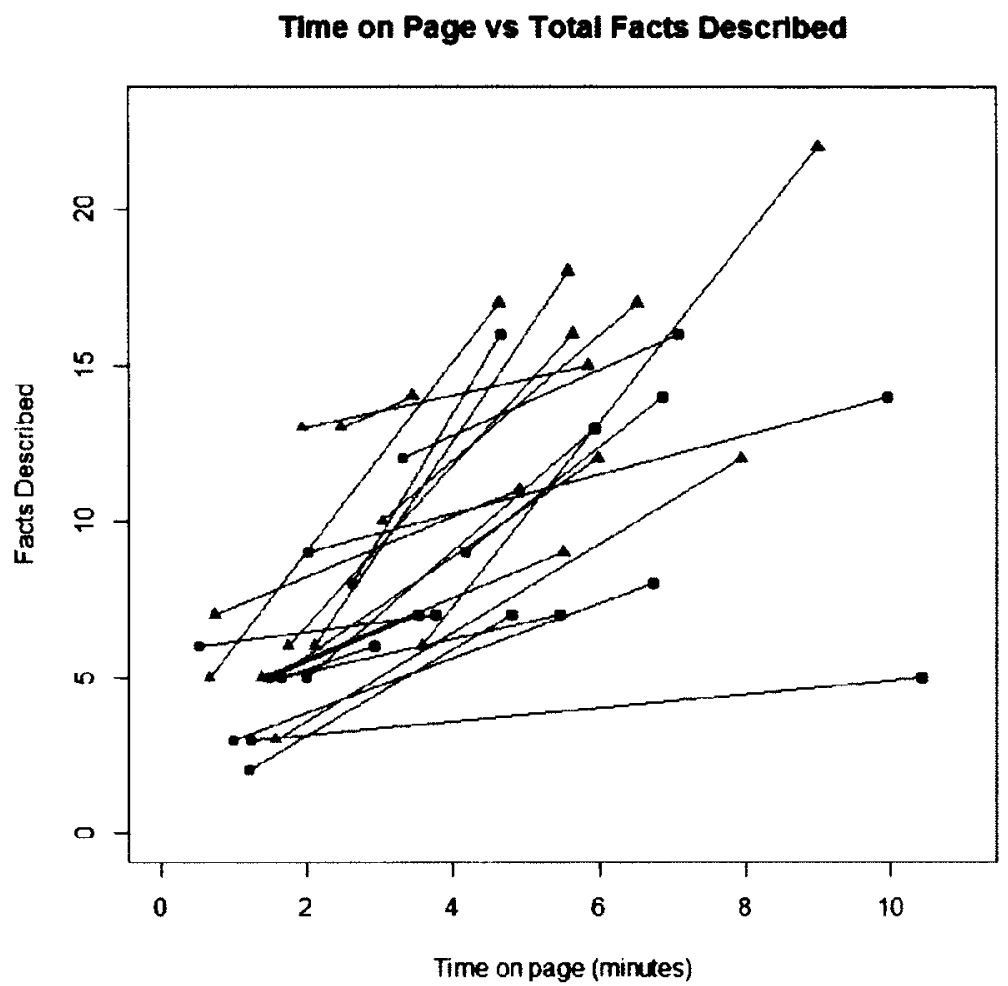

Figure 39: A scatter plot for time spent and all facts described. One line is drawn for each participant, linking their two scores

In all figures, there are more art history participants above the slope than non-art history participants. This is an expected result because the art history participants had some previous knowledge of the subject and will have studied images of this nature.

In this section, we examined the study data relevant to facts recalled in order to see if it supported our hypothesis $\mathrm{H} 2$, that participants will recall more facts and details from the Sketch and Learn images than the textbook-style images. The data included a comparison of the facts recalled, obtained from the audio recording. We also showed scatter plots to help analyze the data and the results supported hypothesis H2. In conclusion, our analysis has shown that the longer the participant spends on the image the more details he will recall. 


\subsection{Questionnaire and Discussion}

The final questionnaire also contains relevant data about the study. No participant had seen all of the images, $23 \%$ had seen most of them, $46 \%$ had seen one or two, and $32 \%$ had not seen any of the images before. Participants who had seen the images were primarily art history students, which was expected. The written response section for this question revealed that the participants had seen some of the images in class, and some from other sources of media including television and art books. A few explained that they had only seen the image of "The Last Supper," a famous image that was only used at the beginning of the study to explain the game.

The next part of the questionnaire featured several questions about the system and the game. Participants were asked to respond using a scale from one to five, one being strongly disagree and five strongly agree. Because of the small sample size the strongly agree and agree scores were combined as were the strongly disagree and disagree. We had included some paired questions, in other words, a negatively phrased and positively phrased version. For example, I enjoyed playing the game; I did not enjoy playing the game. The negative response to the negative phrase and the positive response to the positive phrase were combined and averaged. Also, there were no discrepancies between any of the scores; participants responded with the same viewpoint for both the positive and negative questions.

The questionnaire was recorded anonymously, so we will use it as general feedback to talk about our study. Feedback was very positive; $78 \%$ of participants found the system easy to use and many informally described enjoying the art history study experience. Also, $63 \%$ preferred learning from the game to learning from a textbook.

Although $91 \%$ enjoyed playing the game, only $43 \%$ would play on their own time; however, $87 \%$ would play it to learn material for a class. An interesting study would

\footnotetext{
${ }^{1}$ The percentages may not equal $100 \%$ due to rounding.
} 
be to compare the results from students who have to play this game for a class to students who play the game just because they are interested in art history and enjoy learning through the game. Since $65 \%$ of participants from our study would rather spend more time playing this game than reading from a textbook, a classroom study makes a lot of sense. A study could be run with half the class learning by using only the textbook and the other half using Sketch and Learn.

Participants also answered questions about the sketching mechanic in Sketch and Learn. Almost all, 91\%, said that sketching increased their awareness of certain areas of the art and $61 \%$ said that they felt better while sketching. The responses indicate that using sketching as a learning mechanic is popular among participants in our study and should be considered for more spatial-based educational games.

About $74 \%$ of participants said they would play a similar game just as much or more if it was available in tablet form. Also, $83 \%$ said that they would prefer to use a touchscreen for the sketching part of the game. Those responses provide a strong indication that a tablet version of Sketch and Learn would be of interest.

To summarize responses from the questionnaire, the majority of the participants indicated a preference towards learning from Sketch and Learn to learning from the textbook style and they would also play the game even more if it were available for their phone or tablet. The game opened many of the participants' eyes towards appreciating art, as $85 \%$ of them indicated. The sketching mechanic played a big part in this since most of them enjoyed the sketching while it was increasing their awareness of features in the image.

\subsection{Summary}

Our analysis shows that the data supports hypothesis H1. Participants spent an average of 4.81 minutes on Sketch and Learn images, which was more than three 
times the 1.42 minutes spent on the textbook images. Moreover, participants viewed more of the image, including corners and non-obvious features, in Sketch and Learn and spent more time on the Sketch and Learn images. In addition, we also showed that the participants spent more time on images with higher amounts of glyphs associated with them, supporting hypothesis H1a.

Analysis of the audio data shows that hypothesis $\mathrm{H} 2$ is also supported. Participants recalled more of the content provided, 4.83 facts per image on average, than they had read in the textbook condition (2.74). In addition, the longer a participant spent on the image, the more they were able to recall, as evidenced by a positive slope of 0.53 .

Finally, the majority of participants enjoyed playing the game. They responded positively that they would spend more time and preferred learning through Sketch and Learn. They enjoyed the sketching part of the game and thought it helped them notice more features. This feedback indicates that the Sketch and Learn framework successfully incorporated elements of a good video game.

Some limitations of our study became evident as we analyzed the data. It was an exploratory study, so the sample size of participants was small and the number of images was only 10. Some of the methods chosen to collect the data, the eye tracker and the audio analysis, were time-consuming and labour-intensive; different methods would need to be used for a larger sample size. Despite these limitations, the data was positive enough to warrant future studies of this framework, ideas which will be discussed in the next chapter. 


\section{Chapter 6}

\section{Conclusion}

This thesis began looking at the problem faced by art history students in remembering historical and artistic significance of details of a large number of works of art. Most students are not willing to spend enough time on each image to remember and analyze the details and tend to find the process boring. We speculated that interaction with images using video game mechanics would be a good way to help students. Our thesis was that, by using a combination of effective game mechanics, we could create a learning tool that would extend a person's viewing time on images and would improve their ability to remember the significant facts of those images.

Our review of game design showed that an effective educational game should integrate learning into the gameplay, motivate the players throughout the game and focus their attention on the learning aspects. As well, a truly engaging game should have a flow that keeps players challenged without frustrating them by being too difficult, and a way to reward them as they complete parts of the game. Game mechanics of particular interest to this thesis were prospecting and sketching. Moreover, the concepts of visual attention and memory were key to determining the best way to make sure that the players noticed the significant details and that they remembered them. 
Taking into account the applicable concepts, we designed Sketch and Learn, a framework to create educational art history games. This framework applies prospecting and sketching mechanics to allow the player to interact with our glyph elements. The prospecting mechanic forces players to look at all aspects of the image to search for glyphs that are hidden in it. Once players find these glyphs, they can sketch them to reveal significant features and facts about the image, thereby helping them focus on this information and remember it. We also designed the star elements under the image so that the player can review the completed glyphs and have a clear goal as to how many are left to find and sketch.

In order to test how well our framework met our goal, we developed a game that would allow us to collect data. The game involved Sketch and Learn images interspersed with images that did not employ the game mechanics, but were in textbookstyle. The two key areas for which we collected data were time spent on images and facts recalled from the content. The 25 participants were divided into two groups which viewed opposite images in the Sketch and Learn style and textbook style.

The analysis of the results support our thesis that the game mechanics implemented in Sketch and Learn enable players to spend three times as much time viewing the images than the traditional textbook style. Our results also show that the participants recalled $75 \%$ more of the significant facts about the Sketch and Learn images and pointed out more general details. In general, most of the participants found Sketch and Learn easy to use and enjoyed learning with it.

\subsection{Future Work}

The Sketch and Learn framework has proven to be effective as a educational learning tool. Although the sample size was relatively small, the results are sufficient to merit future study. For instance, a study with a larger selection of images using a much 
larger sample size would allow the researcher to explore various aspects more fully, such as the optimal number of glyphs per image. An ideal study would involve every student in a high school or university class. Half of them would use the game as their primary method of studying the images and the other half would use a textbook, then their performance could be measured by comparing elements such as test scores and time spent on the images.

For a long-term study the game should be enhanced by adding game mechanics such as achievement systems to help motivate players (trophies, leaderboards or other social mechanics). One approach to incorporate achievements and increase learning would be to group like images together. Starting with the most easy concepts, the game would progress to the harder ones; for instance, in art history, you could start with glyphs related to objects and progress to glyphs related to artistic design and concepts. Another enhancement could be tweaking our interface for use on various types of screens, including touch screens, both small and large, would allow students to study in more places. The players in the study showed a strong interest in adapting Sketch and Learn to a mobile interface such as a tablet.

Art history is not the only subject where students must view and understand images, therefore the game mechanics and concepts used in Sketch and Learn can be applied to any subject that uses images to help learn visual concepts or facts. A perfect example of this is biology, where many images are used to explain and show the different organisms and structures. Other examples include anatomy, geography, and any subject where identification is used with both descriptions and images.

In conclusion, the Sketch and Learn framework is a valuable tool for studying images to help students of any language, learn and remember any type of visual information. There is a considerable potential for the framework. We wonder as well whether a similar type of framework could be used for audio information and, in the not-too-distant future, tactile. 


\section{Appendix A}

\section{Art History Images Used in Study}

The following 11 images appear in the study. The images were chosen and provided by Christina Martinez PhD, an instructor of art history at Carleton University.

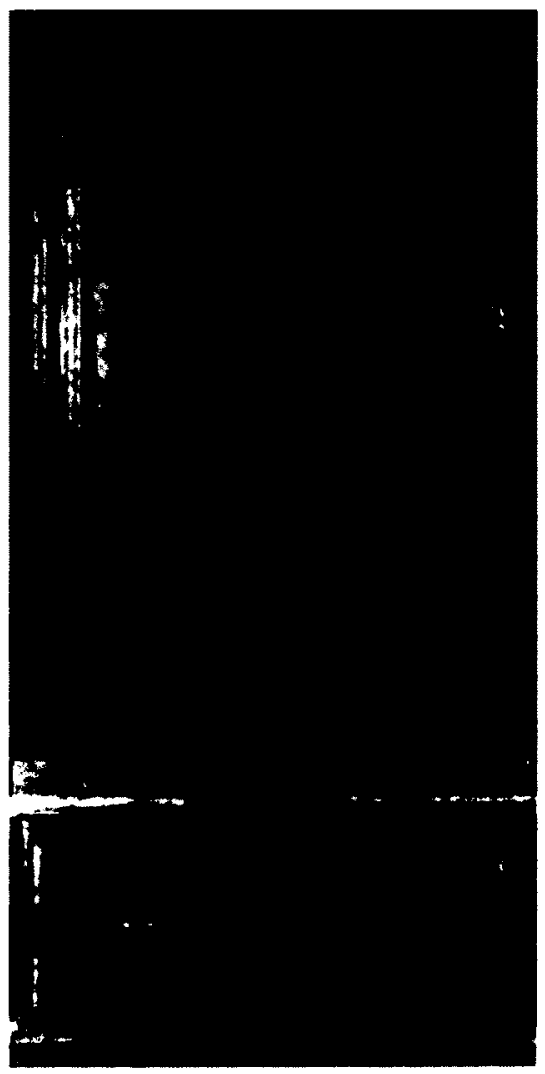

Figure 40: Holy Trinity (Masaccio) 


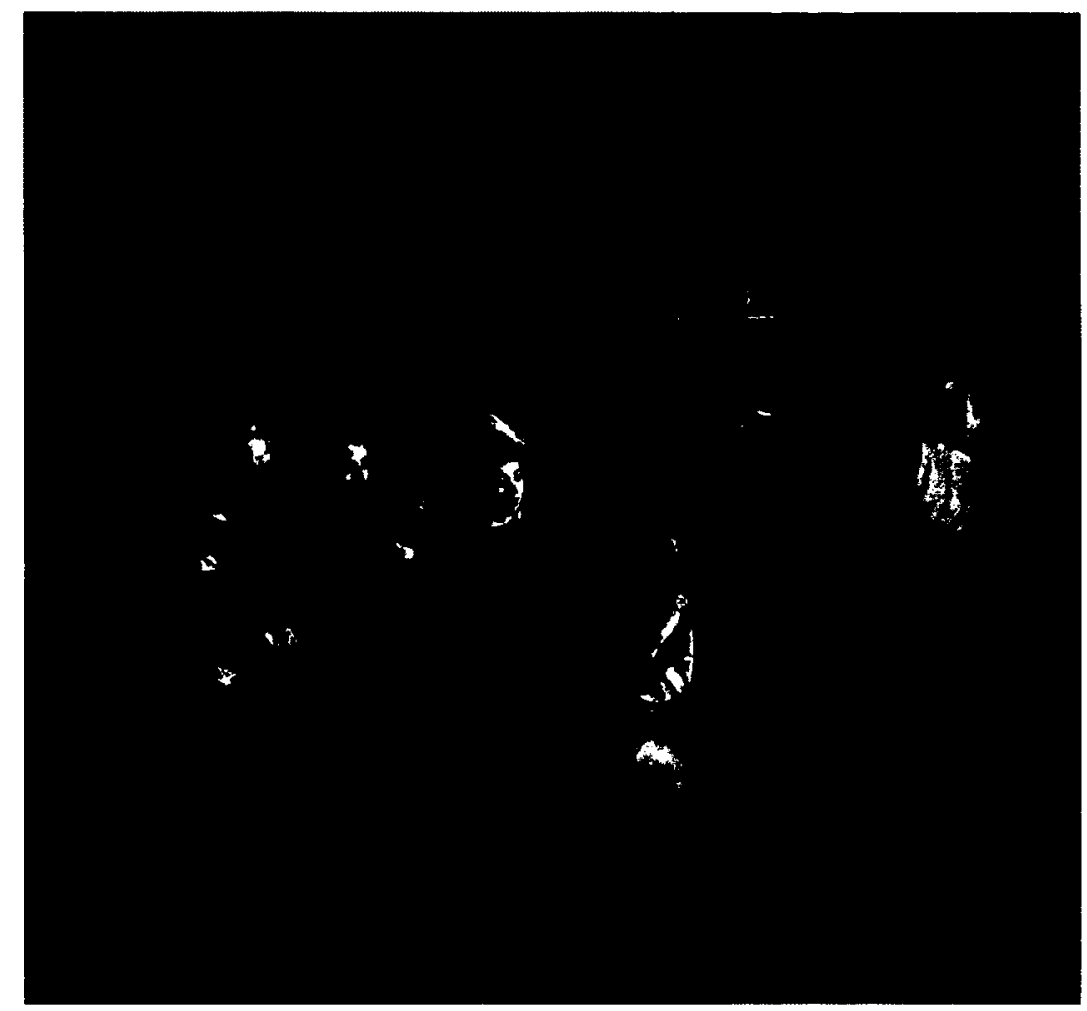

Figure 41: The Calling of St Matthew (Caravaggio)

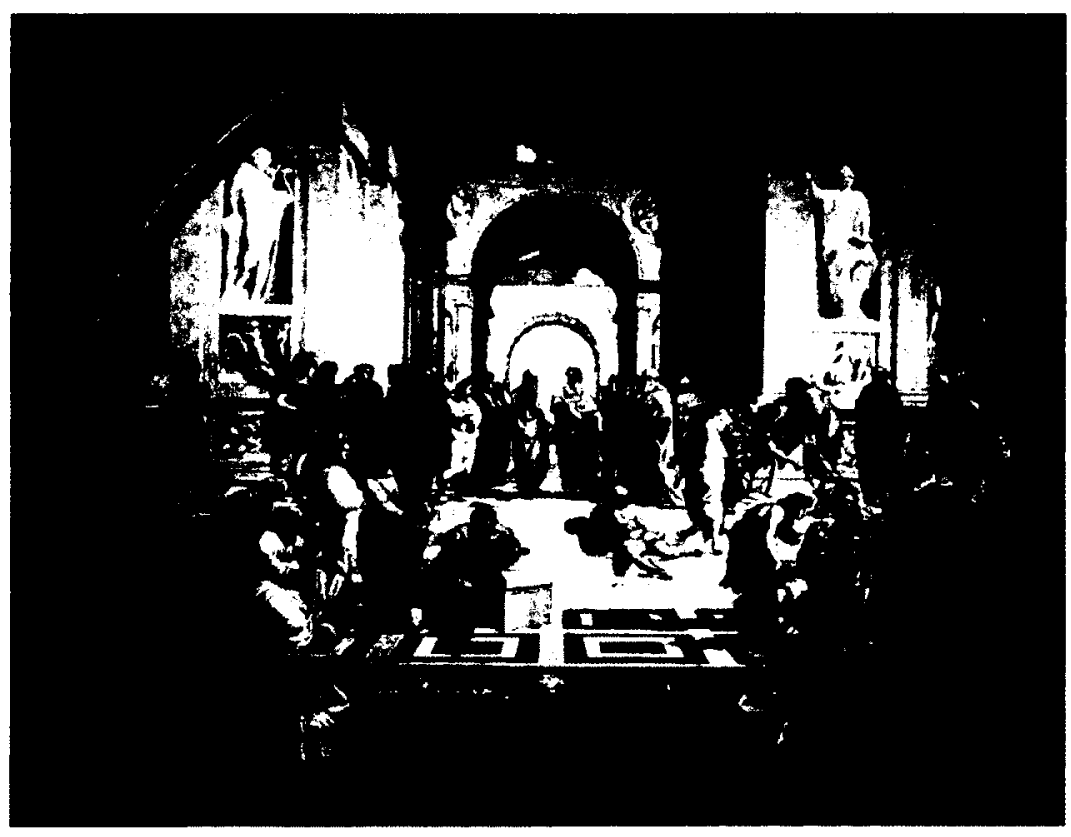

Figure 42: School of Athens (Raphael) 


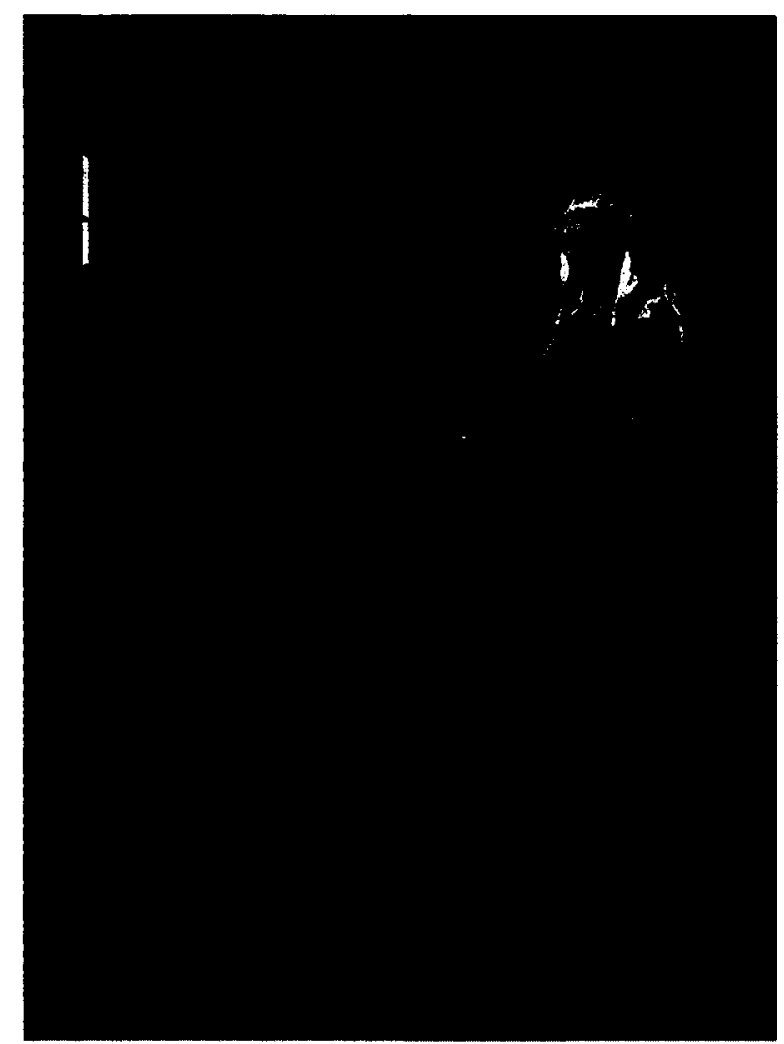

Figure 43: The Arnolfini Portrait (van Eyck)

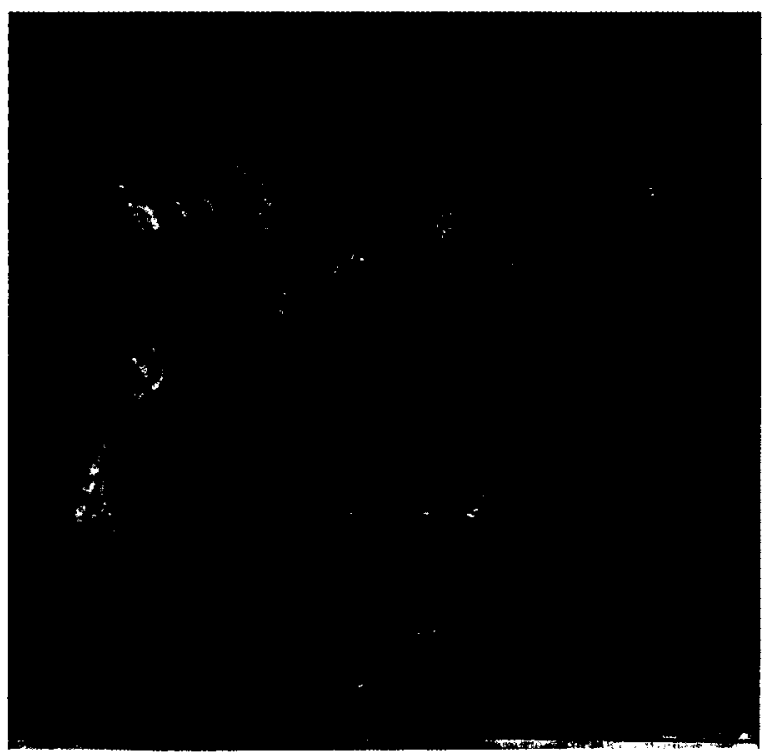

Figure 44: The Ambassadors (Holbein) 


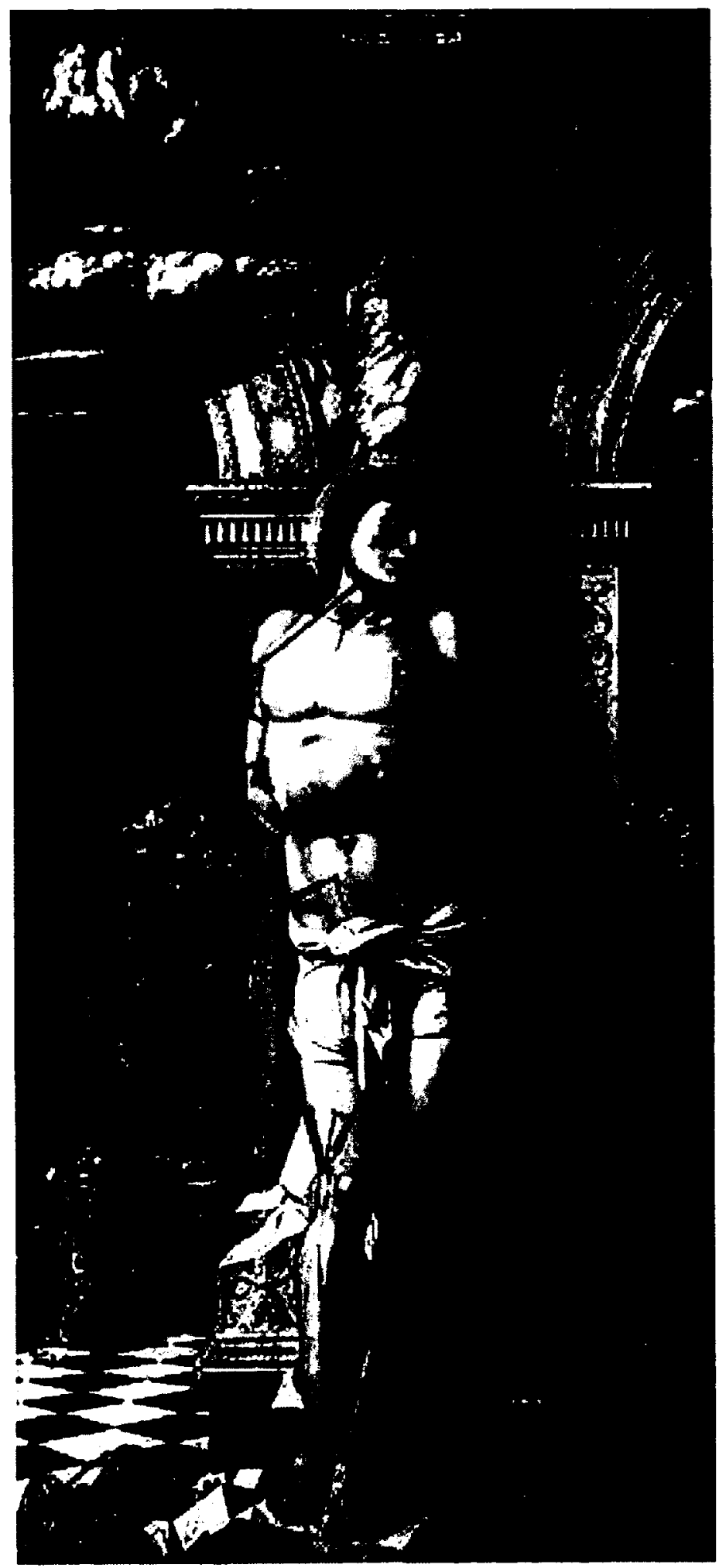

Figure 45: St. Sebastian (Mantegna) 


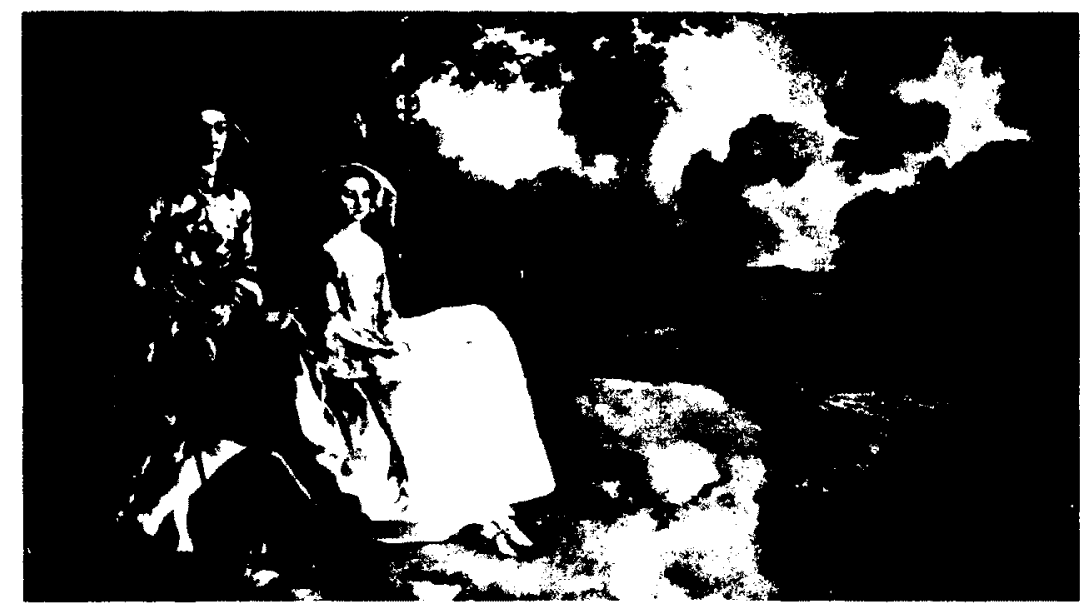

Figure 46: Mr. and Mrs. Andrews (Gainsborough)

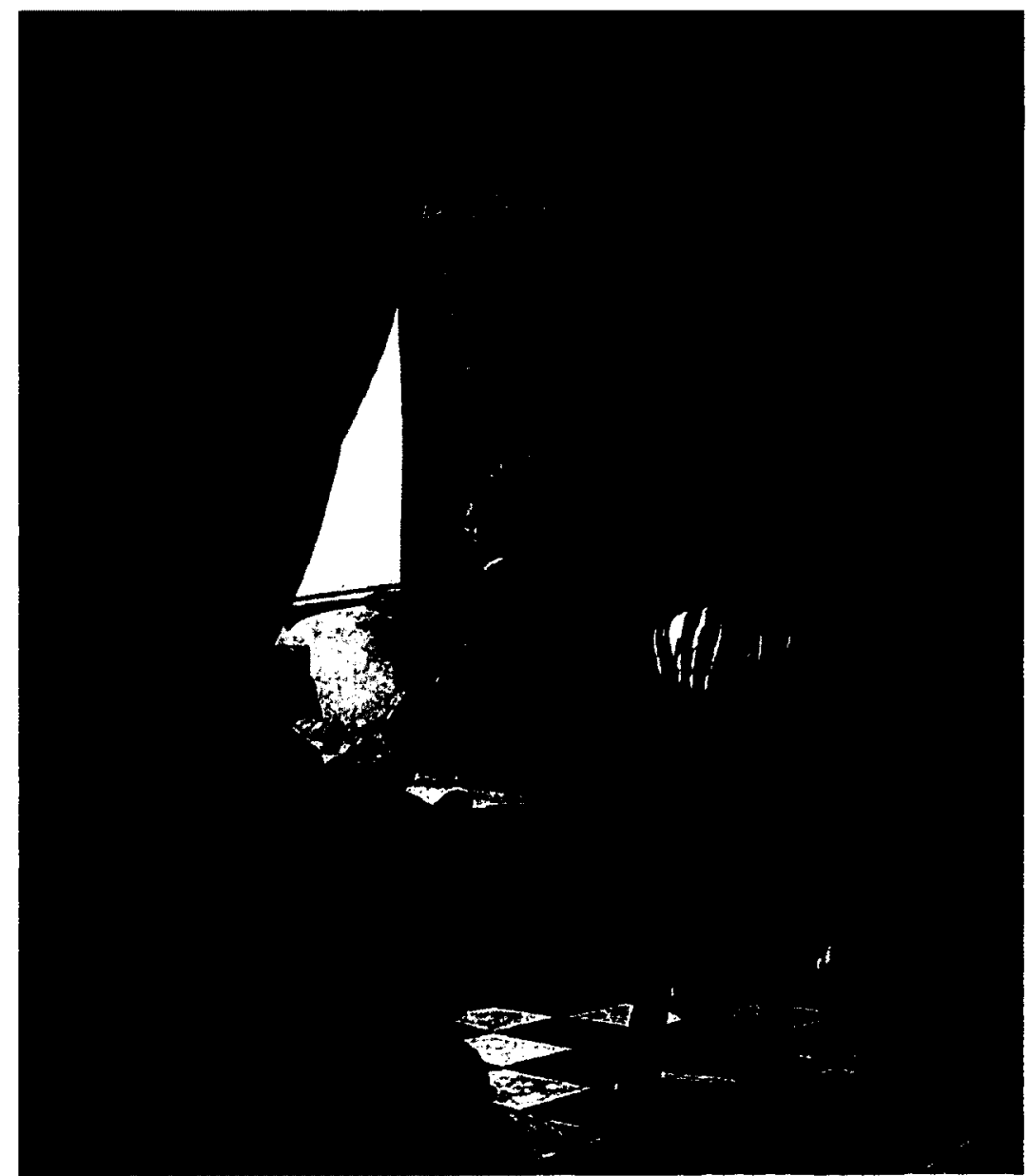

Figure 47: The Art of Painting (Vermeer) 


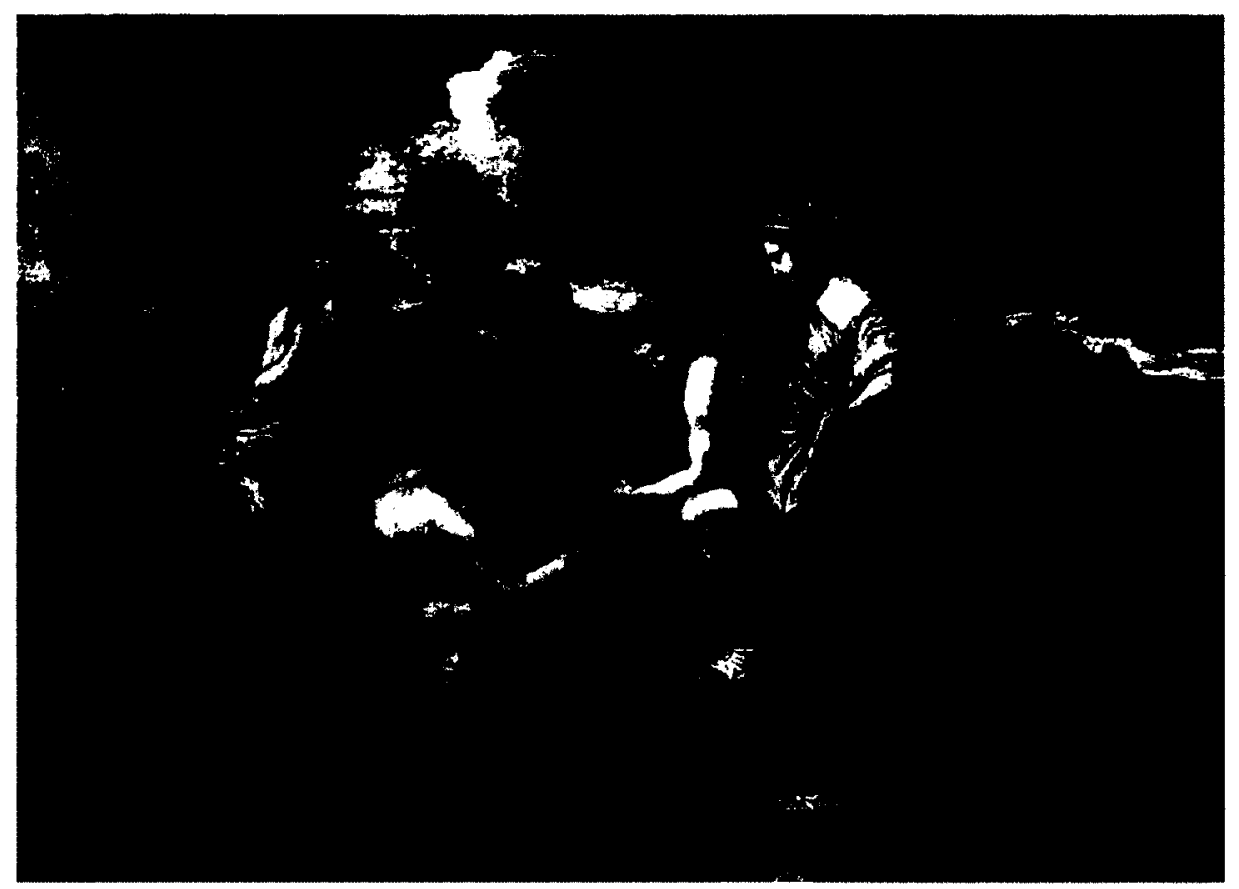

Figure 48: The Arcadian Shepherds (Poussin)

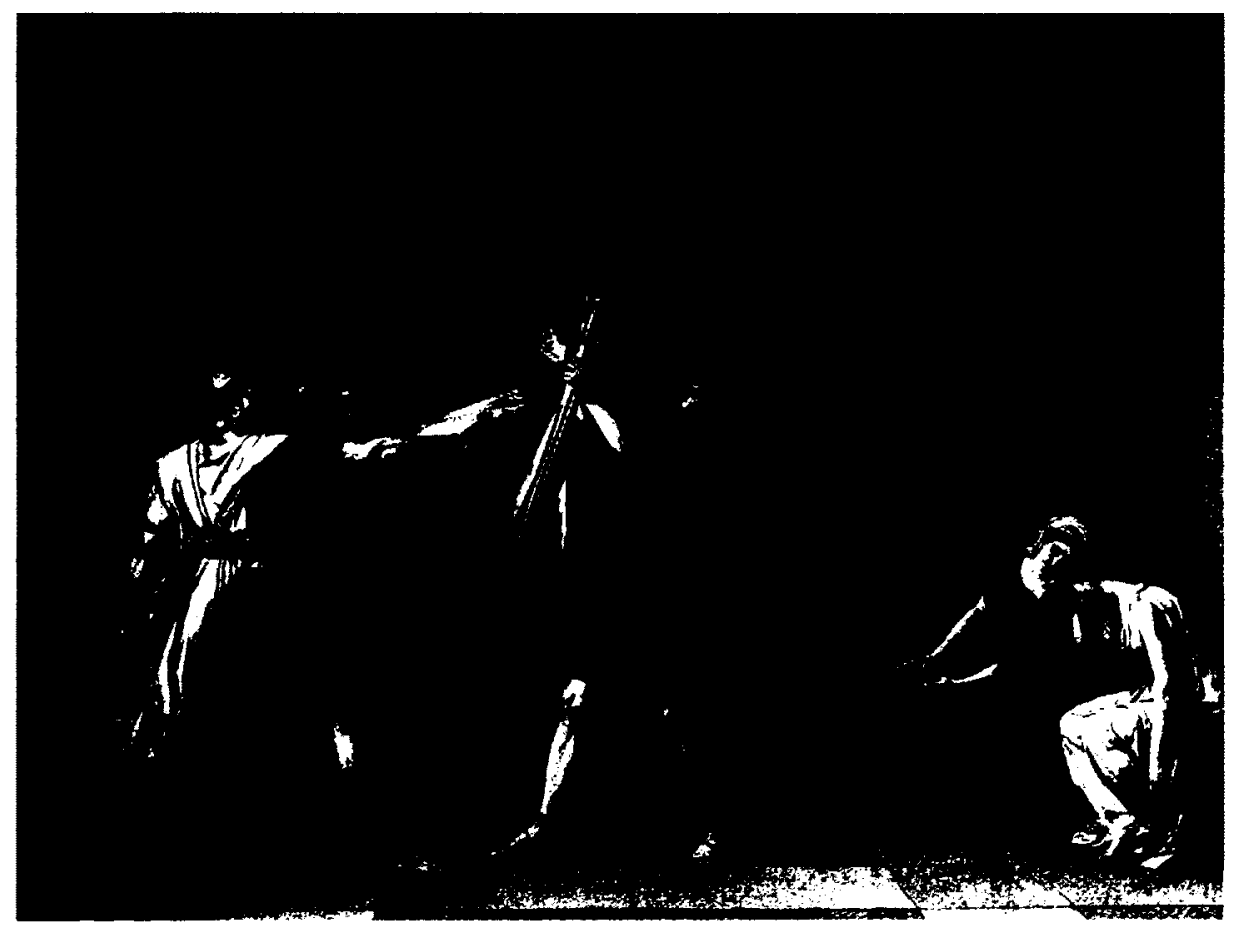

Figure 49: The Oath of the Horatii (David) 


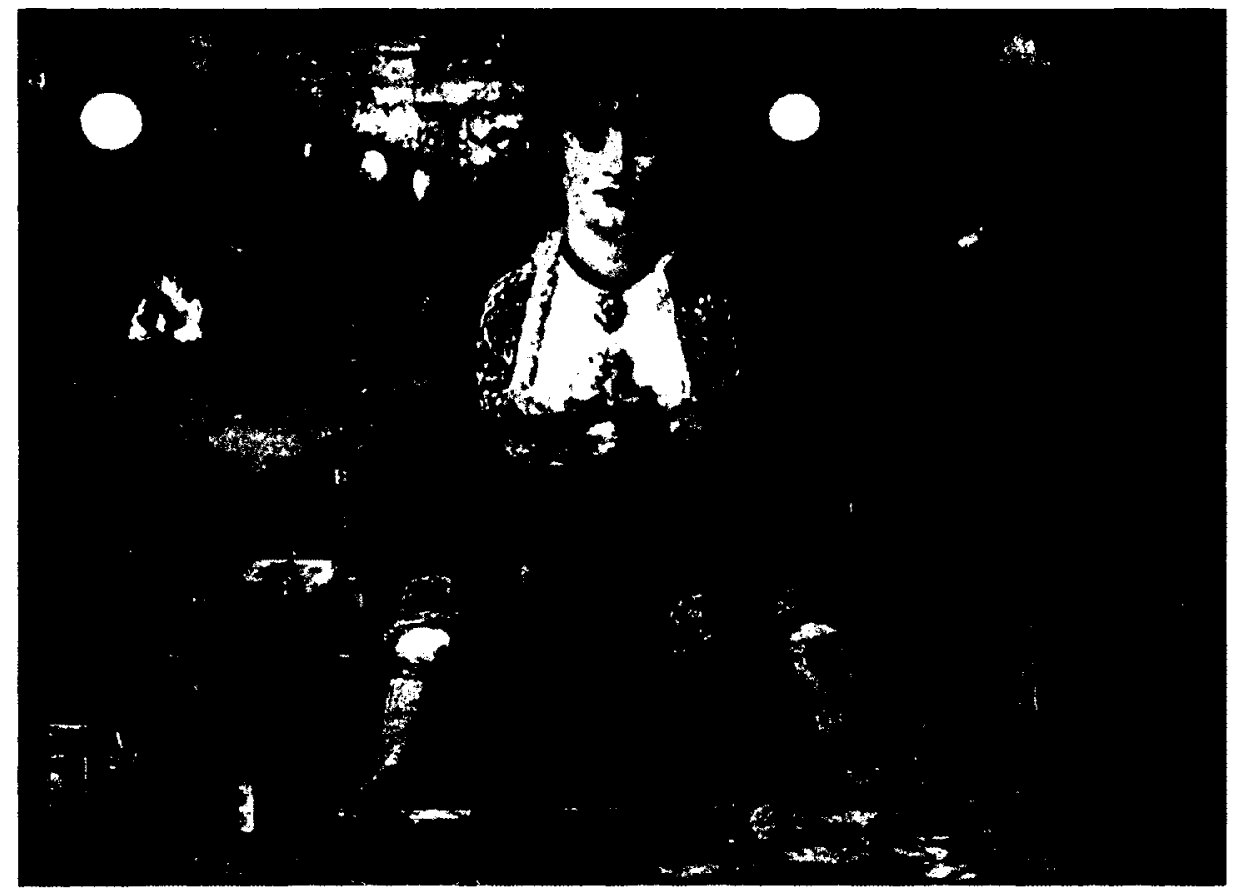

Figure 50: A Bar at the Folies-Bergère (Manet) 


\section{Appendix B}

\section{Questionnaires}

Figure 51: Background Questionnaire

Eackground Information Earthisument

Here is where I will explain about the survey and study.

*1. In what yaar were you born?

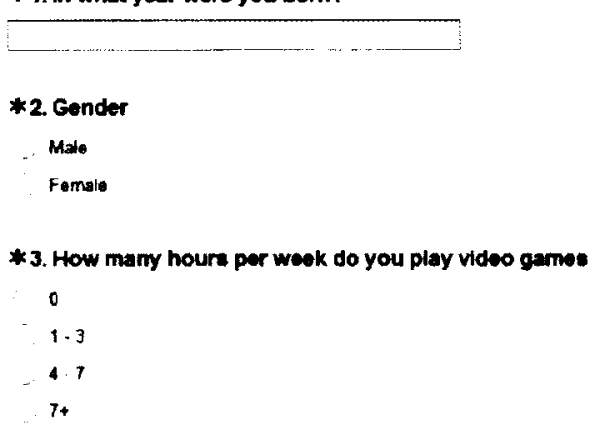

* 4. Why do you play video games? (Pick all that apply to you)

To le arn and gain intellect

$\Gamma$ Entertainment

ГTo pass the time

$\Gamma$ To gel away from ceal life

$\Gamma$ I doni play ndeo games

1 Other (please speciny)

*5. Art History

Provious Knowiedge 
Figure 52: Post-Game Questionnaire

Study Questionnaire

I hope you enyoyed playing the game. Now please complete the final survey.

\section{Have you seen any of these pieces of art before?}
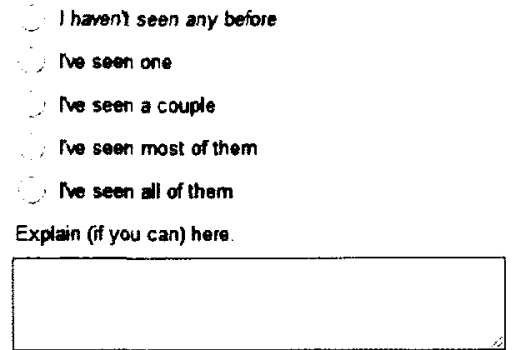

*2. Please click the most approprate anawer.

$\begin{array}{cccc}\text { Strongly disagee } & \text { Disagree } & \begin{array}{c}\text { Neither agree nor } \\ \text { disagree }\end{array} \text { Agree }\end{array}$

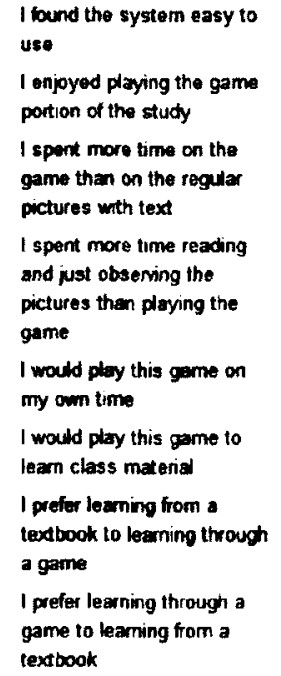




\begin{abstract}
I would spend more time playing this game than

I learned just as much or more from playng the game I did not enjoy playing this game

I would play this just as much or more if it were availatle on a smart phone or lablet

I would profler to use a touchscreen and my finger for the shetching part of the game

The sketching increased my awareness of certain areas of the ant

Simply reading the toxt below the Art was not 28 enjoyatle as unlocking the information through sketching

Reading the blocks of text and associating them to a spot on the art. heiped me understand art betier

I foh better while sketching the at

I fell better just observing the at without skotching Ptaying the game helped me appreciate the art

Playing the game made me
\end{abstract} dislike the art 


\section{List of References}

[1] A closer look at the Mona Lisa - Louvre Museum. http://musee.louvre.fr/oal/joconde/indexEN.html. [Accessed 22/04/12].

[2] About.com. Thumbnail Sketching. http://drawsketch.about.com/library/blthumbnail-sketching.htm. [Accessed 22/04/12].

[3] Activision. Guitar hero. [PS3], 2006.

[4] Electronic Arts. Rock band. [PS3], 2008.

[5] Alice Atkinson-Bonasio. Video Games in Military Training: An Interview with Roger Smith. Instrumentation, pages 1-4, 2008.

[6] J. Banks, II Carson, and N. II. Bl and nicol, dm (2001) discrete-event system simulation. Prentice Hall, 17:18-25.

[7] Richard Bartle. Players Who Suit MUDs. Journal of Online Environments, 1(1):1-25, 1996.

[8] Bioware. Mass Effect 2, 2010.

[9] D E Broadbent. Perception and communication, volume 22. Pergamon Press, 1958.

[10] David Buckingham and Margaret Scanlon. Interactivity and pedagogy in 'edutainment' software. Information technology Education and Society, 4(2):107-126, 2003.

[11] K R Cave and N P Bichot. Visuospatial attention: beyond a spotlight model. Psychonomic bulletin review, 6(2):204-23, 1999.

[12] M M Chun and J M Wolfe. Visual attention. Blackwell Handbook of Sensation and Perception, pages 272-310, 2001. 
[13] Mihaly Csikszentmihalyi. Finding Flow. BasicBooks, 1997.

[14] S De Castell and J Jenson. OP-ED Serious play. J. Curriculum Studies, $35(6): 649-665,2003$.

[15] J M De Oliveira, E E G Echenique, O D G Cruz, and F R Geliz. Games, Gaming and Education. SOCIAL PEDAGOGY, 22(3):129, 2010.

[16] C Delogu. Cognitive factors in the evaluation of synthetic speech. Speech Communication, 24(2):153-168, 1998.

[17] Guillaume Denis and Pierre Jouvelot. Motivation-driven educational game design. In Proceedings of the 2005 ACM SIGCHI International Conference on Advances in computer entertainment technology - ACE '05, pages 462-465, New York, New York, USA, June 2005. ACM Press.

[18] J A Deutsch and D Deutsch. Attention: Some theoretical considerations. Psychological Review, 70(1):80-90, 1963.

[19] Greg Dunham, Ken Forbus, and Jeffrey Usher. nuWar: A prototype sketchbased strategy game. Artificial Intelligence in Interactive Digital Entertainment, 20(10):6, 2005.

[20] Eduweb. A. Pintura: Art Detective. http://www.eduweb.com/pintura/. [Accessed 22/04/12].

[21] Simon Egenfeldt-Nielsen. Beyond Edutainment: Exploring the Educational Potential of Computer Games. PhD thesis, IT University of Copenhagen, Copenhagen, 2005.

[22] Simon Egenfeldt-Nielsen. What Makes a Good Learning Game?: Going beyond edutainment. eLearn, 2011(2):2, 2011.

[23] Charles W Eriksen and James E Hoffman. The extent of processing of noise elements during selective encoding from visual displays. Perception Psychophysics, 14(1):155-160, 1973.

[24] A J Faria, David Hutchinson, William J Wellington, and Steven Gold. Developments in Business Gaming: A Review of the Past 40 Years. Simulation 8 Gaming, 40(4):464-487, 2008.

[25] J Fish and S Scrivener. Amplifying the mind's eye: Sketching and visual cognition. Leonardo, 23(1):117-126, 1990. 
[26] K Forbus, J Usher, and V Chapman. Sketching for military course of action diagrams. In Proceedings of the 2003 international conference on Intelligent user interfaces, pages 61-68. ACM Press, 2003.

[27] Google. Google Art Project. http://www.googleartproject.com/. [Accessed $22 / 04 / 12]$.

[28] Robert D. Griffin. The Biology Coloring Book. Collins Reference, 1986.

[29] Gregory Hennessy. Competitive Simulations: Introducing Corporations to Organisational Learning, volume Microworld. System Dynamics Society, 1994.

[30] R. Hunicke, M. LeBlanc, and R. Zubek. Mda: A formal approach to game design and game research. In Proceedings of the AAAI Workshop on Challenges in Game AI, pages 04-04, 2004.

[31] Mikael Jakobsson. The Achievement Machine : Understanding Xbox 360 Achievements in Gaming Practices. Game Studies, 11(1):1-22, 2011.

[32] The jQuery Foundation. jQuery. http://jquery.com/. [Accessed 01/06/2011].

[33] Microsoft. ASP.NET MVC3. http://www.asp.net/mvc/mvc3. [Accessed 01/06/2011].

[34] Microsoft and Oberon. Minesweeper, 2009.

[35] James S Nairne. Psychology. Wadsworth, Cengage Learning, 5 edition, 2011.

[36] Naughty Dog. Uncharted 3, 2011.

[37] Ulric Neisser and Robert Becklen. Selective looking: Attending to visually specified events. Cognitive Psychology, 7(4):480-494, 1975.

[38] Harold E Pashler. The psychology of attention, volume 41. MIT Press, 1998.

[39] M I Posner and Y Cohen. Components of visual orienting, volume 32. Erlbaum, 1984.

[40] Marc Prensky. Digital game-based learning. Comput. Entertain., 1(1):21, October 2003.

[41] John Resig. Processingjs.org. http://processingjs.org/. [Accessed 01/06/2011].

[42] Katie Salen and Eric Zimmerman. Rules of Play: Game Design Fundamentals. MIT Press, 2003. 
[43] R.C. Schank. Active learning through multimedia. Multimedia, IEEE, 1(1):6978, 1994.

[44] Jesse Schell. The Art of Game Design: A book of lenses, volume 54 of Morgan Kaufmann. Morgan Kaufmann, 2008.

[45] Joanne Sowell. Learning Cycles in Art History. College Teaching, 39(1):14-19, August 2009.

[46] SurveyMonkey. Surveymonkey.com. https://www.surveymonkey.com/. [Accessed 05/05/12].

[47] R Development Core Team. R: A Language and Environment for Statistical Computing, 2011.

[48] Tobii Technology. Tobii Studio 2.2 User Manual, 2010.

[49] Jan Oliver Wallgrün. Qualitative Matching of Spatial Information. in Geographic Information, pages 300-309, 2010. 